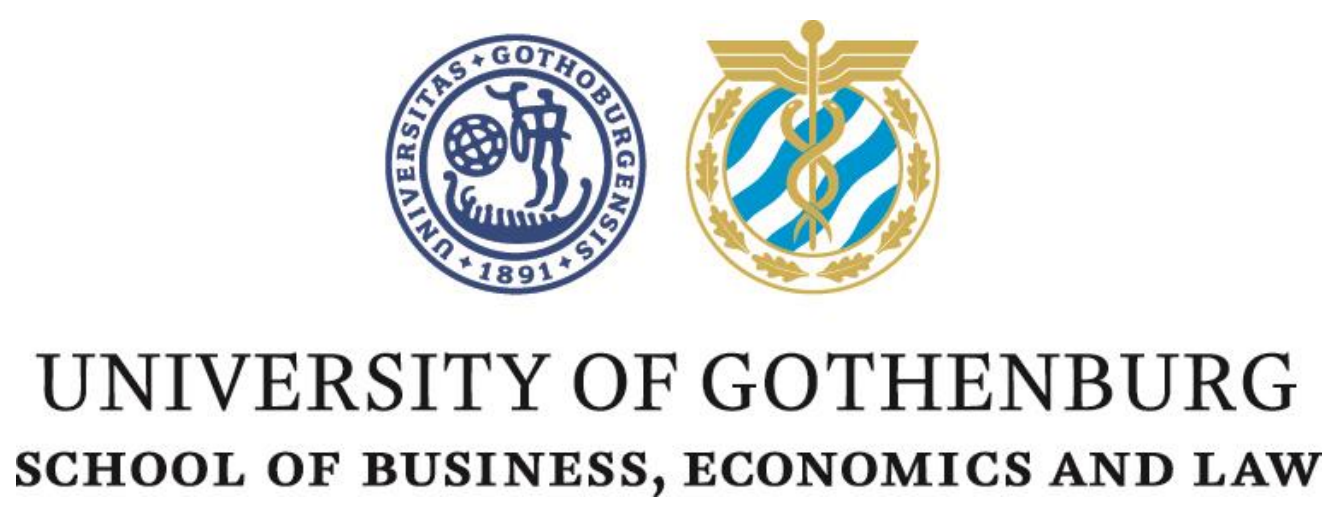

\title{
Are cryptocurrencies homogenous?
}

Frida Gustafsson 


\section{Abstract}

There exist more than 2000 cryptocurrencies today. Although choices of design and target groups vary across cryptocurrencies, current research primarily focuses on Bitcoin. If the differences in design choices makes cryptocurrencies heterogenous then existing research could be less relevant in explaining how the cryptocurrency market works.

Very little is currently known about homogeneity among cryptocurrencies. Therefore, this paper aims to investigate if cryptocurrencies are homogenous. The question of homogeneity among cryptocurrencies is answered via a LASSO-model in which the drivers of returns that have been identified for Bitcoin in the contemporary theoretical framework are applied to a sample of 12 cryptocurrencies, further analysing over time and across design choices of cryptocurrencies.

The results show that cryptocurrencies are heterogenous, apart from some similarities in the impact of technical drivers. The cryptocurrency market is highly integrated with evidence of substitution effects. Further, design choices related to demand and supply among cryptocurrencies can in several cases explain the impact of drivers of return. It is important to consider heterogeneity among cryptocurrencies in order to avoid misinterpretation or misdirected regulation of cryptocurrencies.

Keywords:

Cryptocurrencies, decentralized virtual currencies, LASSO, regulation, homogeneity, heterogeneity, Bitcoin. 


\section{Acknowledgement}

I would like to thank the many people who have inspired and helped me along the way: Joakim Wallenklint at the Swedish Competition Authority for first introducing me to the subject of cryptocurrencies, Research Fellow $\mathrm{PhD}$ Florin Maican from Gothenburg University for invaluable discussions in the early stages of my research idea, my supervisor Associate Professor Elias Bengtsson from Halmstad University for comments that greatly improved the manuscript, always asking the right questions at the right time, and last but not least my family and friends for their endless support. 


\section{Table of content}

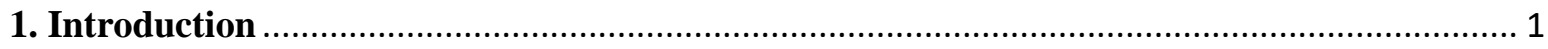

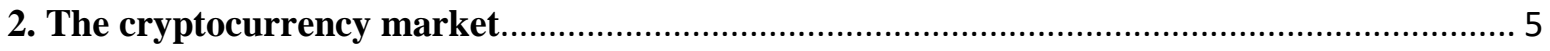

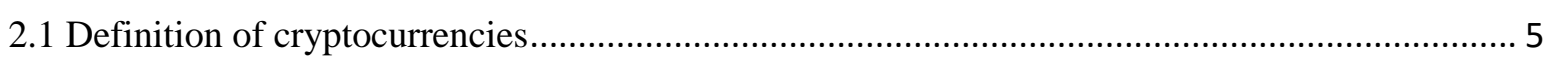

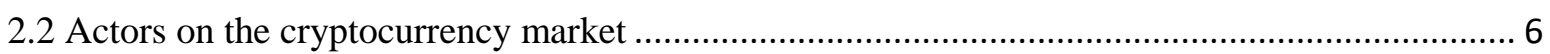

2.3 Differences in design choices among cryptocurrencies .............................................................. 7

3. Theory: Drivers of returns for cryptocurrencies.............................................................. 9

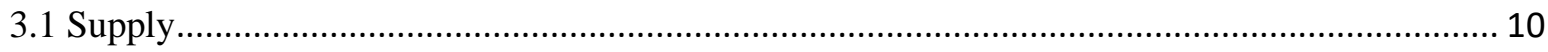

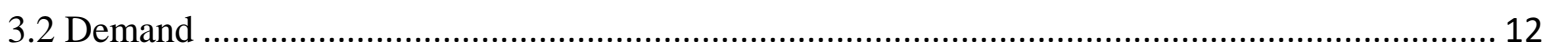

4. Data

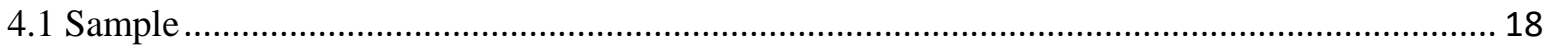

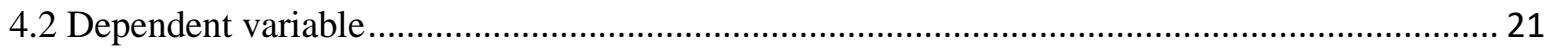

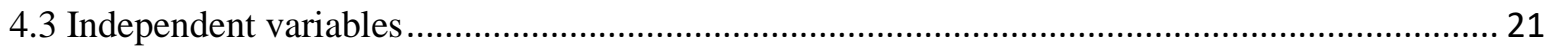

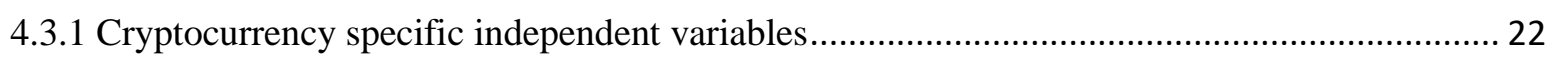

4.3.2 Not cryptocurrency specific independent variables.......................................................... 26

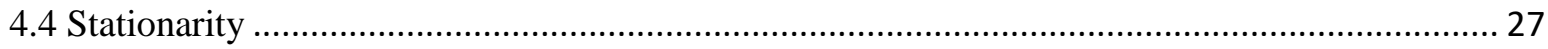

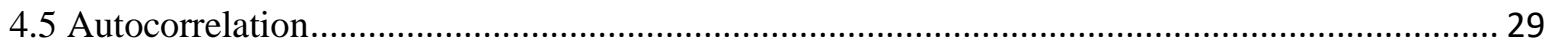

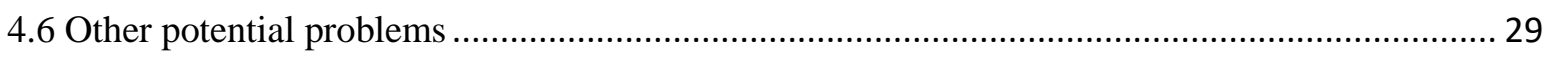

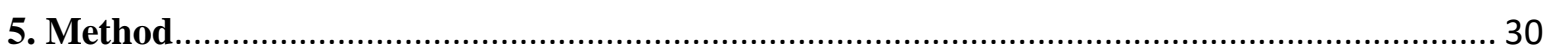

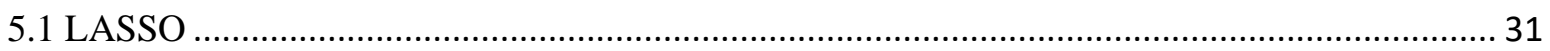

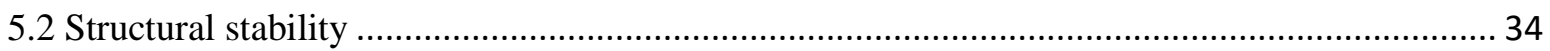

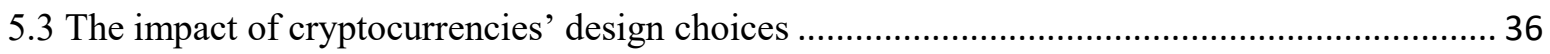

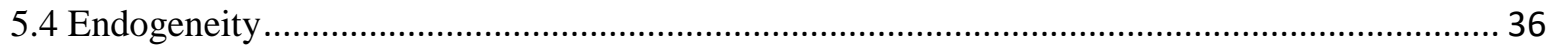

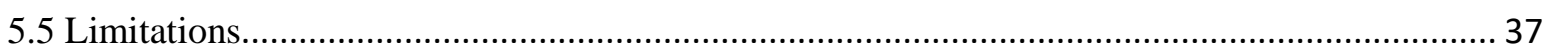

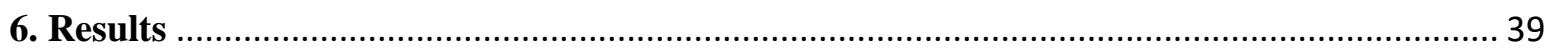

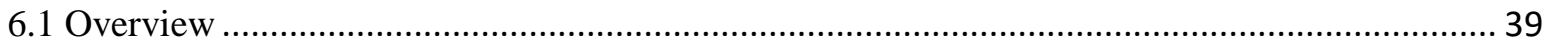

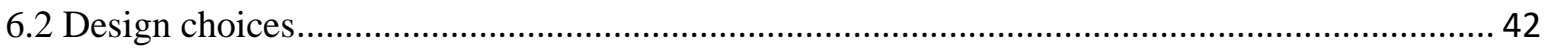

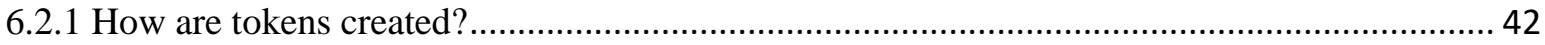

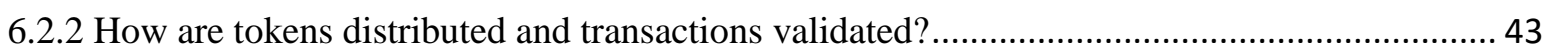

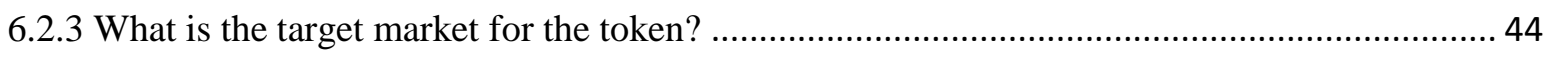

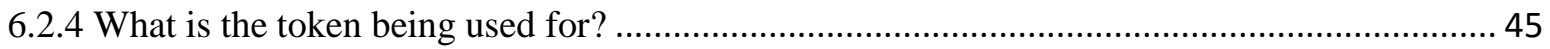

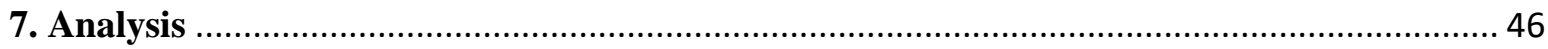

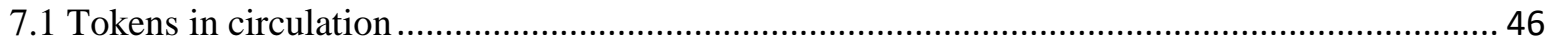

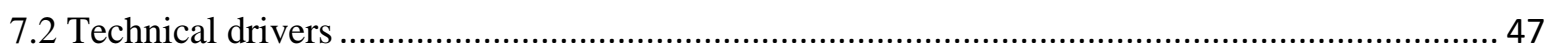

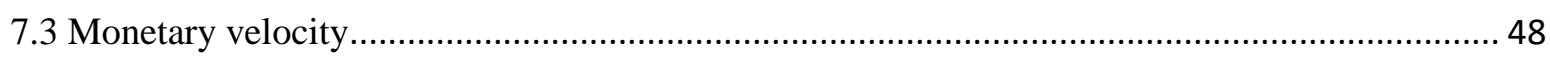

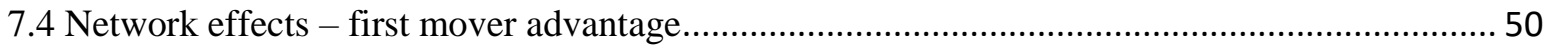

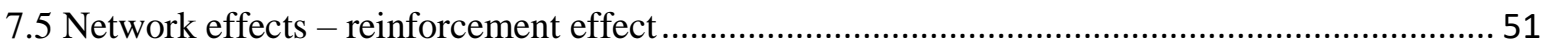




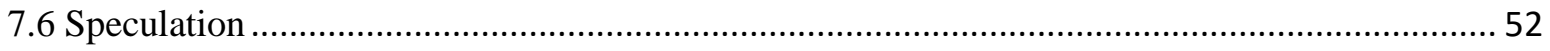

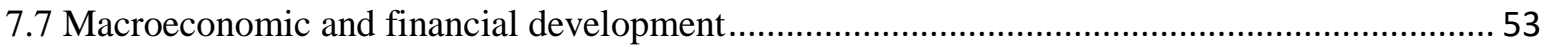

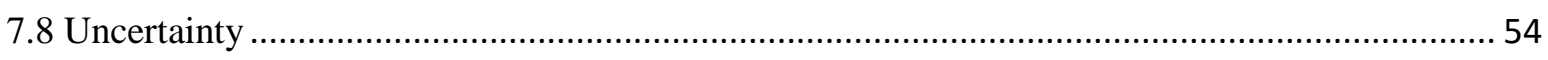

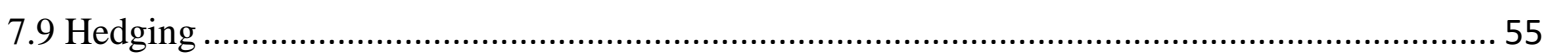

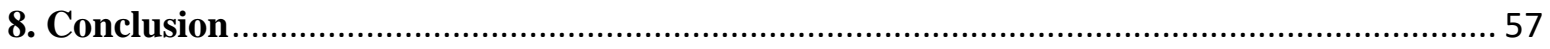

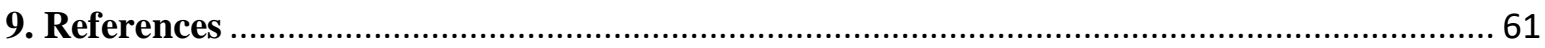

Appendix A: How are tokens created? Results divided by time period .................................... 64 Appendix B: How are tokens distributed and transactions validated? Results divided by time

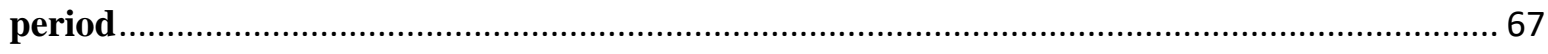

Appendix C: What is the target market for the token? Results divided by time period .......... 70

Appendix D: What is the token being used for? Results divided by time period ..................... 73

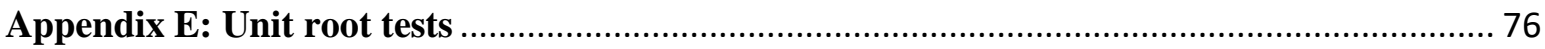




\section{Introduction}

Standard economic goods are priced through the interaction between demand and supply, and these in turn are influenced by macroeconomic or institutional variables on the domestic or international level (Kristoufek, 2013). The demand for a commodity currency is driven by its intrinsic value and its value in future exchanges, for example gold can be used both to create jewellery and for trade (Bouri et al., 2017). In contrast, the value of a fiat currency is based solely on its value in future exchanges and the trust that it will continue to be valuable and accepted as a medium of exchange (Bouri et al., 2017; Kristoufek, 2013). This trust can be enhanced or created by a central issuers actions to guarantee a value for the fiat currency, a role traditionally inhabited by central banks with regards to national currencies.

Cryptocurrencies are fiat currencies that differ from traditional currencies, such as the USD or EUR, in that they do not have a commodity backed value and no central authority guaranteeing its value (ECB, 2012). The rules governing supply are set out at the initial launch of the cryptocurrency. This entails that cryptocurrencies function as a fixed currency with no room for e.g. expansionary monetary policy (Gandal \& Halaburda, 2014). For this reason, the demand for a cryptocurrency is mainly driven by its value in future exchanges (Bouri et al., 2017; Kristoufek, 2013), regardless if its used for speculation or transactions.

There are more than 2000 cryptocurrencies currently listed on Coinmarketcap.com, which makes the large market capitalisation of the top 3 cryptocurrencies remarkable, as seen in figure 1. When comparing the market capitalisation of the top 10 cryptocurrencies in figure 1 , it can be seen that Bitcoin holds a unique position with 52 percent, followed by Ethereum and Ripple (XRP). The large number of cryptocurrencies stands in stark contrast to the large market shares of a select few cryptocurrencies. A potential explanation for both phenomena can be found in theories of network effects ${ }^{1}$.

\footnotetext{
${ }^{1}$ One characteristic that is common to all currencies is the presence of positive network effects and the externalities arising from the effects. Positive network effects mean that the value of a product or service increase with the number of users whereas, for negative network effects the reverse is true.
} 


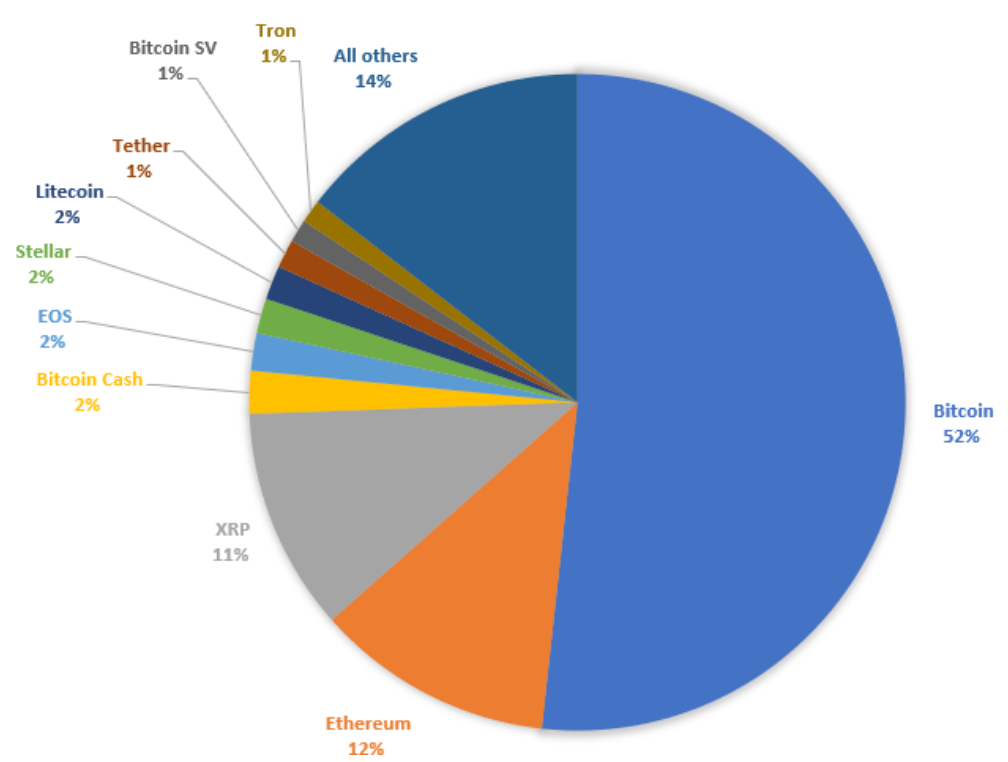

Figure 1: Market shares cryptocurrencies 2019-01-07 (measured by market capitalisation) Source: Coinmarketcap.com (2019-07-21)

Network effects can affect competition in that it makes entry more difficult, giving more influence to incumbent firms with previous established networks. Similar to a traditional fiat currency, the more users a cryptocurrency has, the easier it will be to transform the cryptocurrency into goods and services, thus increasing its value in future exchanges and trust that it will continue to be valuable and accepted as a medium of exchange (Bouri et al., 2017; Kristoufek, 2013). When the cryptocurrency becomes more popular its demand will increase, further adding to its popularity through a reinforcement effect (Gandal \& Halaburda, 2014). The reinforcement effect suggests a movement towards one strong currency, or as implied in figure 1 , a few strong cryptocurrencies.

Further, the sheer number of cryptocurrencies could to some extent be motivated from speculative dynamics connected to the network effects. As the popularity and value of a cryptocurrency increase some people might fear that it is overvalued, which will lead to a substitution effect if they start looking for alternative cryptocurrency investments (Gandal \& Halaburda, 2014). In order to challenge the incumbent firms, the newcomers in a market with strong network effects will often try to distinguish themselves in order to gain an edge in the competition (Gandal \& Halaburda, 2014). There are several traits that can distinguish a cryptocurrency. Cryptocurrencies are similar in that they are based on the distributed ledger technology, but they can display heterogeneity in purpose and design choices (Burnie, 2018). For example, some cryptocurrencies have been implemented to specifically address technical shortcomings of Bitcoin, such as increasing transaction flows or offering a higher level of anonymity (Østbye, 2018; Foley, Karlsen \& Putniņš, 2018). Differences in the designs of 
cryptocurrencies could lead to differences in what influences supply and demand for each cryptocurrency. Differences in aims and which investor group the cryptocurrency targets might also lead to differences in what macroeconomic factors influence the pricing mechanisms and determines the returns of the cryptocurrency.

Cryptocurrencies are, by nature, global and decentralised which might challenge the effectiveness of conventional monetary policy and offer additional risks around the globe. Compared to traditional currencies, cryptocurrencies have hitherto seen relatively little regulation. Several risks have been identified which might require regulatory attention, such as network effects making some cryptocurrencies too-big-to-fail or too-connected-to-fail (Minto et al., 2017), a strong connection to illegal activity and money laundering (ECB, 2015; Chilson, 2018; Foley, Karlsen \& Putniņš, 2018), systemic risk through direct or indirect exposure (Ali, Barrdear, Clews \& Southgate, 2014) and a potential to break the traditional monopoly on money issuance held by Central Banks (Dabrowski \& Janikowski, 2018).

Regardless of the risks regulators try to mitigate, it is important to consider how the cryptocurrency market works ${ }^{2}$. Today, both theoretical and empirical research is narrowly focused on Bitcoin. Only few studies distinguish between currencies, protocols and decentralised applications (Corbet, Lucey, Urquhart \& Yarovaya, 2018). This is a natural consequence since Bitcoin is the by far most well-known cryptocurrency, the most widely adopted cryptocurrency with the longest string of available data.

However, given the potential heterogeneity among cryptocurrencies, an extensive Bitcoin focus might result in lower external validation of the available research. The results found that are applicable to Bitcoin might not be relevant when considering other cryptocurrencies. Further, the large volatility in prices for Bitcoin and other cryptocurrencies makes it necessary to continue conducting research. Results that were obtained in 2016 are probably not the same if obtained in 2018 and should not be considered equal or used in the calculation of averages (Corbet et al., 2018). If cryptocurrencies are homogenous, they should respond similarly to potential regulations implemented, thus facilitating the evaluation and comparison of the

\footnotetext{
${ }^{2}$ When considering regulation for cryptocurrencies it is important to distinguish between regulation of the underlying distributed ledger technology and regulation of the cryptocurrencies themselves. Many of the characteristics that raise concerns around Bitcoin, such as anonymity and extensive energy use, are not necessarily representative for the wider distributed ledger technology (OECD, 2018). Further, they might not be representative for all cryptocurrencies.
} 
potential impact of different types of regulation. However, if cryptocurrencies are heterogenous, their differences could imply that they respond differently to the potential regulations. There is a risk for misinterpretation and misdirected regulation if potential heterogeneity among cryptocurrencies is not considered. Further, if cryptocurrencies are perceived to have high similarities in risk exposure then a high level of interconnectedness could also become problematic as it increases the risk for contagion across the cryptocurrency market.

Currently very little is known about homogeneity among cryptocurrencies. One way to advance knowledge on potential homogeneity among cryptocurrencies is to evaluate if they are homogenous in market characteristics such as supply and demand. Based on the classic economic assumption that all information is known to all investors and reflected in the price of an asset it becomes possible to capture the interactions between supply and demand combined with indirect influences of external factors in their impact on pricing. It is possible to capture a broad image of the cryptocurrency market by looking at prices for cryptocurrencies, in particular which determinants that drive the returns for cryptocurrencies.

The aim of this paper is to evaluate homogeneity among cryptocurrencies. This paper takes the contemporary economic framework and applies the drivers of return that have been previously identified for Bitcoin to a sample of 12 cryptocurrencies, including Bitcoin. Thereby investigating the research question: Are cryptocurrencies homogenous with respect to drivers of returns? If cryptocurrencies are homogenous the same factors will influence, and drive returns for each respective cryptocurrency, including Bitcoin. Thus, this also allows testing whether Bitcoin should be seen as a representative cryptocurrency or not.

The research question is answered via a LASSO-model, a penalized least squares technique that allows some coefficients to shrink towards or be exactly zero, thereby increasing prediction accuracy and creating ease of interpretation across the optimal model of drivers for returns for each cryptocurrency. The returns are further divided into three time periods and analysed over time and across design choices of cryptocurrencies. Finally concluding that, apart from some similarities in the impact of technical drivers, cryptocurrencies are not homogenous.

The generalisability of these results is subject to certain limitations. For instance, some of the cryptocurrency specific determinants of returns evaluated only have available data for cryptocurrencies using a proof-of-work protocol. Further, the lack of cryptocurrencies in some categories have made comparison difficult as it is not possible to find common factors or make 
comparisons in categories which only encompass one cryptocurrency. Another drawback of this study lies in the choice of methodology. The LASSO-approach exploits the variance-bias trade-off, yielding more comparable models but possibly introducing a bias. Several transformations of the data are conducted to decrease the potential bias however exact magnitudes of the drivers of returns should still be interpreted with caution.

The paper is organised as follows, Part 2 offers a definition of cryptocurrencies, an overview of the cryptocurrency market and outlines the differences between cryptocurrencies. Part 3 describes the current economic framework through the theories for drivers of returns that leads up to the choice of the hypotheses tested in this paper. Part 4 describes the data used and transformations made. Part 5 presents the methodology and discusses some potential shortcomings. Part 6 presents the objective findings and their implications, whereas Part 7 provides an in-depth analysis of the individual hypotheses and how they relate to the findings. Part 8 concludes the paper with an outlook for future research.

\section{The cryptocurrency market}

This section describes the cryptocurrency market. Section 2.1 clearly defines the term used to denote cryptocurrencies. Section 2.2 gives an overview of the different actors on the cryptocurrency market and Section 2.3 gives a brief overview of the possible design choices a cryptocurrency can take on, summarized in Figure 1. The potential implications on demand and supply resulting from those design choices are further elaborated on in Part 3.

\subsection{Definition of cryptocurrencies}

Pieters (2017) offer a very clear definition of different types of digital currencies, see figure 2. Based on this Bitcoin, and similar currencies, could formally be described as decentralized virtual cryptocurrencies. Throughout the paper, I will use this definition but denote it with the more commonly known term cryptocurrency. The two terms are often used interchangeably since all cryptocurrencies are also decentralized virtual currencies (Pieters, 2017). Cryptocurrency is also the term used in the majority of the current research papers to collectively denote Bitcoin, Ether, Ripple and other similar decentralized virtual currencies. 


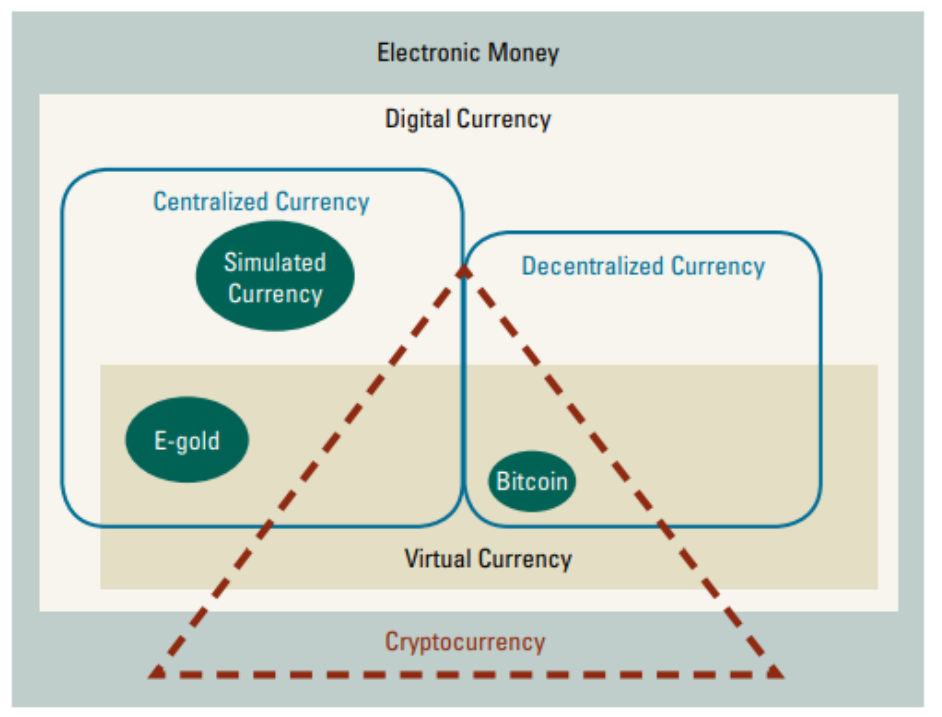

Figure 2: Definition of currencies based on issuer and intended scope of use, transaction verification and technology

Note: Reprinted from "The Potential Impact of Decentralized Virtual Currency on Monetary Policy" by Pieters, G. C., 2017, Available at SSRN: https://ssrn.com/abstract=2976515

\subsection{Actors on the cryptocurrency market}

A cryptocurrency is first created by an inventor, such as Satoshi Nakamoto for Bitcoin. The inventor develops the technical aspects of the network and writes the code (ECB, 2015). Once a cryptocurrency has been launched users can enter the network. Regardless if users choose to purchase cryptocurrencies for their role as means of payment or for speculation there are five ways they can obtain units: 1) purchase, 2) engage in activities that are rewarded with cryptocurrencies, 3) self-generate units through mining, 4) receive units as payment or 5) receive units as a donation or gift (ECB, 2015).

Miners are users that offer computer processing power in exchange for a specific number of units of the cryptocurrency. The computer processing power is necessary to validate the available transactions and adding them to the distributed ledger (ECB, 2015). With more computer processing power, the process of validating becomes faster and more secure since the risk for double-spent or falsely introduced units decrease (ECB, 2015).

Cryptocurrencies are traded on a global scale through trading services and platforms often offered by exchanges (ECB, 2015). Exchanges are usually non-financial companies that accept a wide range of payment options (ECB, 2015). The exchanges quote the exchange rates by which the exchange will buy/sell cryptocurrencies against the main traditional currencies, whereas trading platforms bring together buyers and sellers and allows them to offer and bid 
among themselves (ECB, 2015). Wallet providers offer digital wallets where the users of the cryptocurrency can store their cryptographic key and transaction authentication codes along with the opportunity to initiate transactions and overview of historical transactions (ECB, 2015).

\subsection{Differences in design choices among cryptocurrencies}

The choice of design when implementing a cryptocurrency is written into the initial coding and reflects a variety of potential uses, such as providing a new type of money (e.g. Bitcoin Cash), providing opportunities for a decentralized storage network (e.g. Filecoin) or generally providing a tool for application development (e.g. EOS and Qtum) (Burnie, 2018). Burnie (2018, pp.9-10) provides a comprehensive overview of some of the differences in design choices found among cryptocurrencies, summarized in Table 1. 
Table 1: Design choices among some of the most traded cryptocurrencies

\begin{tabular}{|c|c|c|c|}
\hline \multicolumn{4}{|l|}{ Token supply } \\
\hline \multicolumn{4}{|l|}{ How are tokens created? } \\
\hline \multirow{2}{*}{$\begin{array}{l}\text { Fixed supply } \\
\text { - NEO } \\
\text { - Tron } \\
\text { - Cardano } \\
\text { - Qtum } \\
\text { - Ripple } \\
\end{array}$} & \multirow{2}{*}{\multicolumn{2}{|c|}{$\begin{array}{l}\text { Rise up to cap } \\
\text { - Bitcoin } \\
\text { - Litecoin } \\
\text { - Ethereum Classic } \\
\text { - } \text { Monero } \\
\text { - Bitcoin Cash } \\
\end{array}$}} & $\begin{array}{c}\text { Rise indefinitely } \\
\text { - Ethereum } \\
\text { - Stellar } \\
\text { - } \text { EOS }\end{array}$ \\
\hline & & & $\begin{array}{c}\text { Varies to maintain peg } \\
\text { • Tether }\end{array}$ \\
\hline \multicolumn{4}{|c|}{ How are tokens distributed and transactions validated? } \\
\hline \multicolumn{2}{|c|}{\begin{tabular}{l|l} 
Proof-Of-Work & \\
- & Bitcoin \\
- & Litecoin \\
- & Ethereum \\
- & Ethereum Classic \\
- & Monero \\
- & Bitcoin Cash \\
Run on top of Proof-Of-Work systems \\
- Tron (on top of Ethereum) \\
- Tether (on top of Bitcoin)
\end{tabular}} & \multicolumn{2}{|c|}{$\begin{array}{ll}\text { Voting } & \\
- & \text { Neo } \\
\bullet & \text { EOS } \\
\bullet & \text { Stellar }\end{array}$} \\
\hline \multicolumn{2}{|c|}{$\begin{array}{l}\text { Validators selected } \\
\qquad \quad \text { Ripple }\end{array}$} & \multicolumn{2}{|c|}{$\begin{array}{c}\text { Proof-of-stake } \\
\text { - Cardano } \\
\text { - } \quad \text { Qtum }\end{array}$} \\
\hline \multicolumn{4}{|c|}{ Token demand } \\
\hline \multicolumn{4}{|c|}{ What is the target market for the token? } \\
\hline \multirow{2}{*}{$\begin{array}{l}\text { Generic } \\
\text { - Bitcoin } \\
\text { - Litecoin } \\
\text { - Ethereum } \\
\text { - Ethereum Classic } \\
\text { - Monero } \\
\text { - NEO } \\
\text { - Bitcoin Cash } \\
\text { - Tether }\end{array}$} & & \multicolumn{2}{|c|}{$\begin{array}{l}\text { Business-Oriented } \\
\text { - Cardano } \\
\text { - Ripple } \\
\text { - EOS } \\
\text { - Stellar } \\
\text { - Qtum }\end{array}$} \\
\hline & & \multicolumn{2}{|c|}{$\begin{array}{l}\text { Content Creators on Internet } \\
\text { - Tron }\end{array}$} \\
\hline \multicolumn{4}{|c|}{ What is the token being used for? } \\
\hline $\begin{array}{l}\text { Transaction } \\
\text { - } \text { Litecoin } \\
\text { - Monero } \\
\text { - Bitcoin Cash } \\
\text { - Ripple } \\
\text { - Stellar } \\
\end{array}$ & \begin{tabular}{|c|} 
Hybrid \\
- \\
- Etcoin \\
- Cardano
\end{tabular} & & $\begin{array}{l}\text { Applications } \\
\text { - NEO } \\
\text { - Tron } \\
\text { - } \text { Qtum } \\
\text { - EOS } \\
\text { - Ethereum Classic } \\
\end{array}$ \\
\hline
\end{tabular}

Note: Reprinted from "Exploring the interconnectedness of cryptocurrencies using correlation networks" by Burnie, A., 2018, arXiv preprint arXiv:1806.06632, pp. 9-10

How tokens are created determines the basic supply of the money. A fixed supply means that all the available tokens are created at the time of implementation and that no money creation occurs. A rise up to cap means that money supply increases according to publicly known algorithms up to a certain point. For Bitcoin the cap is set to be a maximum supply of 21 million Bitcoins in 2140 (ECB, 2015). If a currency is implemented with increasing money supply but 
no cap, then it has the possibility to rise indefinitely. There are also some examples of cryptocurrencies trying to increase price stability by implementing a peg against one of the major traditional currencies, e.g. Tether pegged to the USD.

Distribution of tokens and validation of transactions contribute to the supply of the cryptocurrency. With a proof-of-work system the miners compete for the right to validate transactions with their computational power whereas with a proof-of-stake system the validators are chosen based on their stakes in the system, e.g. total number of own coins. A system with selected validators means adding trusted miners to the system that are the only one's able to validate transactions. Who the trusted miners are can also be determined through voting in which all account holders vote for their ideal mining candidates.

The target market for the cryptocurrency is determined by how they define their intentions in their whitepaper, the first presentation of the cryptocurrency written by the inventor, or in other public communication. Business-oriented cryptocurrencies are those who are explicitly seeking commercial applications for their technology, such as for payments (Ripple, Stellar), for developing applications (Qtum, EOS) or for both (Cardano) (Burnie, 2018). Generic cryptocurrencies target a broader audience, motivating both business and non-business use of their token (Burnie, 2018). One noTable cryptocurrency that did not fit either category is Tron that specifically targets content creators online (Burnie, 2018).

Further the cryptocurrencies could be divided by token functionality, if the token is designed and used mainly for transacting value, if tokens are designed to enable new development to applications or if tokens use a combination of the two functions (Burnie, 2018).

\section{Theory: Drivers of returns for cryptocurrencies}

One way of understanding the cryptocurrency market is to investigate the interaction between demand and supply that gives the prices on the market and their resulting returns. This is based on the classic economic assumption that all information is known to all investors and reflected in the price of an asset. As prices tend to fluctuate, often with upwards or downwards moving trends, daily returns can often offer more comparable measures of development over time. The homogeneity of cryptocurrencies will be evaluated by comparing the relative importance of drivers of returns. If cryptocurrencies are homogenous the same factors will influence, and drive returns for each respective cryptocurrency, including Bitcoin. Thus, this would further allow testing whether Bitcoin should be seen as a representative cryptocurrency. Bitcoin could be 
representative if each cryptocurrency is influenced by the same variables as Bitcoin, at best with equal strength in effect.

This rests on a simple logic, either cryptocurrencies are homogenous or not. The aim of this paper is to investigate this by attempting to prove that cryptocurrencies are homogenous with respect to drivers of returns. Further, if this holds true then Bitcoin could be seen as a representative cryptocurrency. Thus, the overarching null and alternative hypotheses are defined as:

$\mathrm{H}_{0}$ : Cryptocurrencies are homogenous with respect to drivers of returns. Bitcoin could be seen as a representative cryptocurrency.

$\mathrm{H}_{\mathrm{A}}$ : Cryptocurrencies are non-homogenous, i.e. heterogenous, with respect to drivers of returns. Bitcoin should not be seen as a representative cryptocurrency.

By looking at several potential drivers of returns identified in the current Bitcoin literature the null hypothesis can be evaluated to see if it holds for all aspects. Therefore, the overarching null hypothesis has been divided into several sub-hypotheses, ranging from $\mathrm{H}_{1}$ to $\mathrm{H}_{9}$, to test different variables. The null hypothesis could be said to hold true if cryptocurrencies display homogeneity in all sub-hypotheses and in the relative importance of the variables tested.

Section 3.1 describes the sub-hypotheses that relates to the supply of a cryptocurrency whereas 3.2 describes the sub-hypotheses that relates to the demand. The alternative hypotheses have been omitted in the following theory section for simplicity and ease of reading.

\subsection{Supply}

The supply function of a cryptocurrency is either fixed or evolves according to publicly known rules set out at the launch of the cryptocurrency, such as the mining algorithms used to control the supply of Bitcoin (Kristoufek, 2013). Essentially, a cryptocurrency functions as a fixed currency with no influence from central authorities (Gandal \& Halaburda, 2014). Since the supply is publicly known and predefined or fixed in the long run, the supply of a cryptocurrency becomes exogenous to its own pricing mechanism, in contrast to gold that is endogenous in that a higher price might lead to more intensive mining (Bouri et al., 2017). Factors such as technical productivity and number of the cryptocurrency in circulation contribute to the pricing mechanism in defining an equilibrium between demand and supply for each cryptocurrency. A small and finite supply of cryptocurrencies combined with a high confidence in the design will 
be reflected in high demand, which in turn leads to higher prices and large price fluctuations (Bianchi, 2018).

\subsubsection{Tokens in circulation}

Differences in how tokens are created contributes to supply in that it sets the initial framework for the number of tokens in circulation. For cryptocurrencies with a fixed supply the tokens in circulation is a fixed number. For cryptocurrencies that rise up to cap the number of tokens in circulation will continue to rise up to a certain point and for cryptocurrencies with no cap this increase is continuous. Thus, unless the supply is fixed it will continue to increase over some time. According to traditional economic theory increases in supply should lead to lower prices, ceteris paribus. Thereby, the number of tokens in circulation could be expected to have predictive power over the returns for cryptocurrencies that do not have a fixed supply. Ciaian, Rajcaniova and Kancs (2016) found that the number of Bitcoins has a negative impact with regards to determinants of Bitcoin prices. Li and Wang (2017) tested determinants of the Bitcoin exchange rate towards the USD and found that increases in Bitcoin supply had a significant effect in the early Bitcoin market (when the Mt. Gox exchange was still open) but not in the later market (after the closing of Mt. Gox in 2014). Polasik, Piotrowska, Wisniewski, Kotkowski \& Lightfoot (2015) found no significant effect of changes in supply on Bitcoin returns. If cryptocurrencies are homogenous the choice of design for token creation should not create any variation across the cryptocurrencies tested, instead they should all follow the potential negative impact on returns that an increased supply suggests for Bitcoin.

$\mathrm{H}_{1}$ : Variations in number of tokens in circulation do not create any differences in determinants of returns for cryptocurrencies.

\subsubsection{Technical drivers}

Technical choices in the protocol design for cryptocurrencies are reflected in how tokens are distributed and transactions validated. Although the underlying distributed ledger technology is common for all cryptocurrencies the exact technical choice on how to reach consensus in transactions might vary. The choice of technical drivers and characteristics of the cryptocurrencies, such as technology used and limitation of quantity produced, could contribute to a positive value for a cryptocurrency (Dwyer, 2015). Thus, it could also contribute in driving returns, particularly if the technologies differ in their ability to provide efficient transactions. Technical drivers can be proxied by a cryptocurrency's hash rate, a measure of how much computational power a cryptocurrency's network consumes in order to generate a new block in 
the blockchain (Bouoiyour \& Selmi, 2015). The higher the hash rate the more likely a new block will be mined. Cryptocurrencies with higher hash rates decrease the time it takes for transactions to be approved and added to the blockchain and thereby become more attractive for trade. Bouoiyour and Selmi (2015) found a significant positive long run impact from increases in hash rate on log of Bitcoin prices. In a later analysis they use quantile regression and find a significant positive effect on the Bitcoin price index when prices are in bear state (the lower quantiles) but no significant effect for bull state (upper quantiles) or for the full sample (Bouoiyour \& Selmi, 2017).

$\mathrm{H}_{2}$ : Variations in hash rate do not create any differences in determinants of returns for cryptocurrencies.

\subsection{Demand}

Demand for a cryptocurrency is driven by its value in future exchanges (Bouri et al., 2017; Kristoufek, 2013). The utility of holding a cryptocurrency can be influenced by several factors, such as its perceived usefulness for transactions or a high confidence in the design and future increased value of the cryptocurrency.

Since the aim and use of cryptocurrencies vary, it becomes likely that changes in for example macroeconomic variables affect returns differently. A cryptocurrency mainly used for transactions should likely be positively affected by a favourable financial environment, for example its demand should increase as the stock indices improve. In contrast, if a cryptocurrency is used as a hedge against macroeconomic instability, its demand should increase as stock indices deteriorate or as volatility indices increase.

\subsubsection{Monetary velocity}

The monetary velocity of a cryptocurrency describes the rate at which money is exchanged in the cryptocurrency economy, i.e. how fast a cryptocurrency passes from one owner to the next. This offers a measure of the perceived usefulness of a cryptocurrency, and thus a higher monetary velocity could contribute to an increase in demand for a cryptocurrency. This might be particularly important depending on the target market of the token. Business-oriented cryptocurrencies that explicitly seek to provide commercial applications might benefit more from a higher perceived usefulness of the cryptocurrency, as compared to cryptocurrencies targeting content creators online. For cryptocurrencies such as Tron, a higher monetary velocity might still increase demand but it is likely not as critical as for cryptocurrencies targeting 
business applications. Cryptocurrencies that target generic markets could end up on both ends of the spectrum. The monetary velocity can be proxied by for example output volume (Bouoiyour \& Selmi, 2015), number of transactions performed (Polasik et al., 2015) or days destroyed per transaction (Ciaian et al., 2016). Bouoiyour \& Selmi (2015) find no significant impact from monetary velocity on the logarithmized Bitcoin prices. In their later analysis, they discover a significant positive effect when looking at the whole sample and a negative effect that is significant for the bear state quantiles (Bouoiyour \& Selmi, 2017). Polasik et al. (2015) shows that monthly change in number of Bitcoin transactions have a significant positive impact on Bitcoin returns. Ciaian et al. (2016) find no significant impact of days destroyed on Bitcoin prices.

$\mathrm{H}_{3}$ : Variations in monetary velocity do not create any differences in determinants of returns of cryptocurrencies.

\subsubsection{Network effects - first mover advantage}

One particular characteristic of the cryptocurrencies market is a strong presence of positive network effects. Currencies traditionally display large positive network effects since a currency is more useful when more people adopt it, and the more popular it becomes the more easily it can attract new users (Gandal \& Halaburda, 2014). Markets with strong network effects often have unsTable competition since larger networks have an advantage which increases as new users join or switch from existing products (Waldman \& Jensen, 2016). Further, the presence of network effects often creates multiple equilibria, either a lot of people join the platform because they expect a lot of people to join or the exact opposite could happen, that people do not join since they expect few others to join (Gans \& Halaburda, 2013). This tipping effect makes it difficult for smaller networks to stay in business unless they display distinguishing characteristics and thereby the presence of network effects in a market affects competition since it makes entry more difficult (Waldman \& Jensen, 2016).

Given the presence of network effects, it becomes relevant to look at the date of implementation for each cryptocurrency. Generally, we would expect older cryptocurrencies to have taken a larger share of the market and therefore be perceived as more useful for future transactions, thus the increased demand should lead to higher returns. The previously accounted literature has not used date of implementation as an explanatory variable when studying cryptocurrencies' returns. A decision likely made due to the difficulty in including a variable measuring a specific date without introducing multicollinearity into the model. One alternative way to look at the 
matter is offered by Li and Wang (2017). They split their analysis of Bitcoin exchange rate towards the USD into early market (2011-01-01 - 2013-12-31) and late market (after closing of Mt.Gox, measured from 2013-12-31) (Li and Wang, 2017). This allows them to evaluate differences between early years of implementation and later years where Bitcoin had become more established. Comparing across different time periods offers a way to circumvent the potential problems of multicollinearity and still evaluate development over time for a specific cryptocurrency. It also makes it possible to divide and compare the sample of cryptocurrencies by their date of implementation.

$\mathrm{H}_{4}$ : Variations in date of implementation do not create any differences in determinants of returns of cryptocurrencies.

\subsubsection{Network effects - reinforcement effect}

Another consequence of network effects could be a reinforcement effect seen in the movement towards one strong currency, a "winner-takes-all” race (Gandal \& Halaburda, 2014). Alternatively, if speculation is the main focus of investors, the network effects could give rise to a substitution effect. For example, as Bitcoin become more popular and more expensive, users could begin to worry that it might be overvalued and look for an alternative cryptocurrency investment (Gandal \& Halaburda, 2014). By including lagged values of returns for some of the major cryptocurrencies a proxy for these potential effects could be captured. The lead in a "winner-takes-all" race should be negatively impacted by increases in returns of other cryptocurrencies. Cryptocurrencies presented as alternative investment should be positively impacted by increases in returns of the lead of the "winner-takes-all" race. The network effects could help to explain the different roles of cryptocurrencies by highlighting their position as for example incumbents in the market. Consequently, heterogeneity in cryptocurrencies market capitalisation could lead to different positions, with Bitcoin likely leading a potential "winner-takes-all" race whereas other cryptocurrencies could be more affected by a potential substitution effect.

$\mathrm{H}_{5}$ : Lagged values of returns for competing cryptocurrencies do not create any differences in determinants of returns of cryptocurrencies. There is no distinguishable move towards either a “winner-takes-all" race or a substitution effect. 


\subsubsection{Speculation}

Part of what drives demand in the cryptocurrency market is the expected profits of holding a cryptocurrency and selling it later, the speculative element (Cheah \& Fry, 2015; Baur, Dimpfl $\&$ Kuck, 2018). Depending on the intended use for the token, the speculative element might be more or less important. Cryptocurrencies used for transactions might be held for a shorter time and thereby the speculative element could be less noticeable. For cryptocurrencies intended for use in applications, a new development might have a long-term perspective before it pays off, which in turn offers potential profits of holding the currency. The intrinsic value of a cryptocurrency is zero since no underlying asset value exists (Cheah \& Fry, 2015). Thus, the price of a cryptocurrency is driven by the investor's faith in future expected profits and perpetual growth, which in turn makes investor sentiment an important variable (Kristoufek, 2013). This is proxied by investor attention through measures of Google searches (Kristoufek, 2013; Bouyoir and Selmi 2015), possibly further decomposed into above or below trend values (Panagiotidis, Stengos \& Vravosinos, 2018).

Kristoufek (2013) proxies investor attention by Google and Wikipedia searches. He finds that the number of searches is significant in explaining Bitcoin prices but that the effect varies depending on the state of the Bitcoin market, the effect is positive when Bitcoin prices are above their trend value and negative when prices are below the trend value (Kristoufek, 2013). Panagotidis et al. (2018) find similar significant effects of Google searches on Bitcoin returns above and below trend, the strongest effect being with above trend values. Bouyoir and Selmi (2015) use total number of Google searches as a proxy for investor attention, which yields a significant positive effect on the logarithmized Bitcoin price in the short run but no effect in the long run. In a later analysis, they choose to use Google search queries for two countries of particular interest for their paper, India and Venezuela (Bouyoir \& Selmi, 2017). They find a significant positive effect on Bitcoin price index when the market is functioning around normal and bull regimes (Bouyoir \& Selmi, 2017). Li and Wang (2017) use Google searches and number of Twitter tweets and find a significant positive effect for both measures on the exchange rate of Bitcoin towards the USD in the early market period, whereas in the late market period the effect only remains for Google searches. Ciaian et al. (2016) find similar results using views on Wikipedia, a significant positive impact on Bitcoin prices but no effect in the long run. One potential explanation for this is that the information found on Wikipedia is at a basic level which in the long run has already become known to most users (Ciaian et al., 2016). Polasik et al. (2015) find a significant positive impact on Bitcoin returns from both percentage 
increase in Google searches and percentage increase in number of articles mentioning Bitcoin.

$\mathrm{H}_{6}$ : Variations in level of speculation, proxied by investor attention, do not create any differences in determinants of returns of cryptocurrencies.

\subsubsection{Macroeconomic and financial development}

The decentralized nature of cryptocurrencies implies that traditional macroeconomic drivers of supply and demand for a currency do not directly influence the pricing mechanism. To offer a comparison, a traditional currency could adjust the exchange rate to accommodate changes in GDP, unemployment and financial status in the home country of the central issuer. For the USD, macroeconomic factors in the US are essential in explaining the price of the currency. However, in the case of cryptocurrencies the lack of a central issuer means that the potential impact of macroeconomic factors and financial indicators work in a more indirect manner.

One example of a potential channel could be if favourable macroeconomic and financial development led to increased use of cryptocurrencies in trade and exchanges and thereby strengthened its demand, which in turn could have a positive impact on returns (Bouri et al., 2017). This effect could be stronger for cryptocurrencies targeting individual users and transactions as these might be more influenced by general movements on the market. Ciaian et al. (2016) find that global macroeconomic and financial developments, such as the Dow Jones index and oil prices, do not significantly impact Bitcoin prices in the long run. Similarly, Bouyoir and Selmi (2017) show a short run positive impact from the Shanghai market index on the logarithmized Bitcoin prices but the effect does not remain in the long run. Panagotidis et al. (2018) find a positive effect of increases in the Nikkei index and oil prices on Bitcoin returns, however the overall effects from the stock markets are mixed.

$\mathrm{H}_{7}$ : Variations in macroeconomic and financial development do not create any differences in determinants of returns of cryptocurrencies.

\subsubsection{Uncertainty}

An alternative channel is offered by the possibility to use cryptocurrencies for hedging against traditional asset classes (Dyhrberg, 2016; Baur et al., 2015). This is likely a more advanced strategy and perhaps not something the average individual investor will attempt. If some cryptocurrencies are perceived as less related to the traditional markets, they could be used to hedge against this uncertainty. For example, an ability to hedge global uncertainty could 
increase demand for a cryptocurrency when the traditional economy experiences a downturn, thereby raising the price and increasing the return. Panagotidis et al. (2018) find a significant negative effect on Bitcoin returns from increases in both Chinese and British uncertainty indices. Bouri et al. (2017) show that for short-term frequencies Bitcoin display some hedging capacities when the market is in bull regime, but in the bear regime they find a significant negative impact from the world uncertainty index on Bitcoin returns. Bouoyir and Selmi (2017) find a positive effect on the Bitcoin price index from the US volatility index when the market is in normal mode and from the British volatility index when the market is in bull state.

$\mathrm{H}_{8}$ : Variations in global and regional uncertainty do not create any differences in determinants of returns of cryptocurrencies.

\subsubsection{Hedge}

Traditional assets used for hedging include gold and fiat currencies. Thus, increases in those variables could signal a move towards more hedging which in turn could also increase demand for cryptocurrencies that are perceived as more suitable to use for hedging. Panagotidis et al. (2018) discover that the gold price and exchange rates have a positive effect on Bitcoin returns but only the gold price is significant. Bouoyoir and Selmi (2015) find no significant effect of gold price on the logarithmized Bitcoin prices. However, in their later analysis they find a negative effect of gold price on the Bitcoin price index when the market is in bear state, and a significant negative effect of the Chinese Yuan when the market is in bull state (Bouoyoir \& Selmi, 2017).

$\mathrm{H}_{9}$ : Variations in financial development for assets traditionally used for hedging do not create any differences in determinants of returns of cryptocurrencies. 


\section{Data}

This section describes the data used and the variables selected in order to test the hypotheses. Section 4.1 describes the sample of cryptocurrencies used with a final list in Table 4. Section 4.2 describes the dependent variable, cryptocurrency's return, and transformations conducted. Section 4.3. describes the independent variables, divided into cryptocurrency specific variables in section 4.3.1 (summary statistics in Table 6) and variables that remain the same regardless of which cryptocurrency is investigated in section 4.3.2 (summary statistics in Table 7). Section 4.4 describes regression diagnostics and unit root tests.

\subsection{Sample}

The subset of cryptocurrencies was selected if they had a large userbase relative to other cryptocurrencies, along the lines of Burnie (2018). For a cryptocurrency with a smaller userbase there will be a smaller number of buyers and sellers at any given point in time. Thus, sellers and buyers might need to adjust their prices to encourage sufficient demand and supply for their desired transaction. This increases the volatility of prices for smaller cryptocurrencies, which in turn will be driven by random noise (Burnie, 2018).

A userbase can be either measured by market capitalisation or liquidity (Burnie, 2018). First a list of the recent top ten cryptocurrencies, ranked by either market capitalisation or liquidity (measured as total exchange volume), were compiled, see Table 2 and 3. Comparing the resulting selection with the selection made by Burnie (2018) it was found to be almost identical, with the exception of Bitcoin SV (BSV) and Monero (XMR). Bitcoin Satoshi's Vision (i.e. SV) was created out of a hard fork on Bitcoin Cash on November 15, 2018 (https://www.coindesk.com/price/Bitcoin-sv, 2019-07-21). Its recent date of implementation as compared to the other cryptocurrencies offers too little data for comparison and thus Bitcoin SV was omitted from the sample. Monero has dropped from the top ten, to ranking 11 when looking at market capitalisation. As it still remains among the high rankings it was included in the sample to increase the number of cryptocurrencies evaluated. 
Table 2: Top ten cryptocurrencies ranked by total exchange volume (USD)

\begin{tabular}{r|lll} 
& CRYPTOCURRENCY & ABBREVIATION & TOTAL EXCHANGE VOLUME (USD) \\
\hline $\mathbf{1}$ & Bitcoin & BTC & $21.04 \mathrm{~B}$ \\
$\mathbf{2}$ & Ethereum & ETH & $8.15 \mathrm{~B}$ \\
$\mathbf{3}$ & Litecoin & LTC & $3.35 \mathrm{~B}$ \\
$\mathbf{4}$ & EOS & EOS & $2.18 \mathrm{~B}$ \\
$\mathbf{5}$ & Ripple & XRP & $1.64 \mathrm{~B}$ \\
$\mathbf{6}$ & Bitcoin Cash & BCH & $1.5 \mathrm{~B}$ \\
$\mathbf{7}$ & Ethereum Classic & ETC & $578.74 \mathrm{M}$ \\
$\mathbf{8}$ & Tron & TRX & $549.68 \mathrm{M}$ \\
$\mathbf{9}$ & Stellar & XLM & $495.90 \mathrm{M}$ \\
$\mathbf{1 0}$ & NEO & NEO & $474.53 \mathrm{M}$
\end{tabular}

Source: https://www.coindesk.com/data (2019-07-21)

Table 3: Top ten cryptocurrencies ranked by market capitalisation (USD)

\begin{tabular}{r|lll}
\multicolumn{2}{c}{ CRYPTOCURRENCY } & ABBREVIATION & MARKET CAPITALISATION (USD) \\
\hline $\mathbf{1}$ & Bitcoin & BTC & $190.45 \mathrm{~B}$ \\
$\mathbf{2}$ & Ethereum & ETH & $24.28 \mathrm{~B}$ \\
$\mathbf{3}$ & Ripple & XRP & $13.18 \mathrm{~B}$ \\
$\mathbf{4}$ & Litecoin & LTC & $6.26 \mathrm{~B}$ \\
$\mathbf{5}$ & Bitcoin Cash & BCH & $5.87 \mathrm{~B}$ \\
$\mathbf{6}$ & EOS & EOS & $4.36 \mathrm{~B}$ \\
$\mathbf{7}$ & Bitcoin SV & BSV & $3.17 \mathrm{~B}$ \\
$\mathbf{8}$ & Tron & TRX & $1.99 \mathrm{~B}$ \\
$\mathbf{9}$ & Stellar & XLM & $1.82 \mathrm{~B}$ \\
$\mathbf{1 0}$ & Cardano & ADA & $1.62 \mathrm{~B}$
\end{tabular}

Source: https://www.coindesk.com/data (2019-07-21)

The final sample of cryptocurrencies thus include 12 cryptocurrencies, listed with date of implementation and abbreviation in Table 4. For simplicity in Tables and ease of reading, the abbreviations listed in Table 4 are continuously used throughout the analysis.

For an overview of the data used and its sources see Table 5. The cryptocurrency specific data were retrieved from CoinMetrics 2019-04-30. The data used covers the period 2013-10-02 to 2018-04-01. For more details and motivation for the time periods selected, see Section 5.2. Data that is not in a daily frequency or that is in a 5-day frequency has been linearly interpolated to a 7-day frequency. The starting date is chosen to exclude the early-adoption phase of the first cryptocurrencies. The early adoption phase of cryptocurrencies mainly consist of data on Bitcoin prices, few transactions, low prices and small price fluctuations. Thus, for a comparison of homogeneity among cryptocurrencies the early adoption phase offers little additional information and its specific characteristics risk distorting the results. All variables are transformed to logarithmic first differences so that they are stationary and their coefficients 
comparable, more details in Section 4.5. After transformation all variables were found to be stationary, i.e. I(0), more details in Appendix E.

Table 4: Cryptocurrencies used and their abbreviations

\begin{tabular}{lll}
\hline Cryptocurrency & Implementation & Abbreviation \\
\hline Bitcoin BTC & $2009-01-03$ & BTC \\
\hline Litecoin LTC & $2011-10-07$ & LTC \\
\hline Ripple XRP & $2013-01-02$ & XRP \\
\hline Monero XMR & $2014-04-18$ & XMR \\
\hline Stellar XLM & $2014-08-05$ & XLM \\
\hline Ethereum cl ETC & $2015-07-30$ & ETC \\
\hline Ethereum ETH & $2015-07-30$ & ETH \\
\hline NEO & $2016-09-09$ & NEO \\
\hline EOS & $2017-06-20$ & EOS \\
Bitcoin cash BCH & $2017-07-23$ & BCH \\
\hline Tron TRX & $2017-08-28$ & TRX \\
\hline Cardano ADA & $2017-09-24$ & ADA \\
\hline
\end{tabular}




\subsection{Dependent variable}

Previous research has used returns measured on a monthly (Polasik et al., 2015), daily (Panagotidis et al., 2018; Balcilar Bouri, Gupta \& Roubaud, 2017) or various frequencies basis (Bouri et al., 2017). It can also be measured as prices (Ciaian et al., 2016; Kristoufek, 2013), sometimes logarithmized (Bouyoir \& Selmi, 2015), exchange rate of the cryptocurrency towards the USD (Li and Wang, 2017) or through a daily price index (Bouyoir \& Selmi, 2017). This paper uses returns measured on a daily basis, transforming the daily prices to a first difference log return of each cryptocurrency.

This paper uses the available daily (7-day) pricing data in USD for each cryptocurrency. In order to ensure stationarity and comparability of the data a log transformation is conducted as well as a first difference calculation of returns:

$$
y_{i t}=\Delta \ln \left(p_{i t}\right)=\ln \left(p_{i t}\right)-\ln \left(p_{i, t-k}\right)
$$

Where $p_{i t}$ is the price of a cryptocurrency, $y_{i t}$ is the first difference log return of cryptocurrency $i$ where $i=1,2 \ldots, N$, at time t. $k$ is the number of lags specified and for the first difference returns $k=1$, i.e. a lag of one day.

\subsection{Independent variables}

The independent variables can be described as a vector of the form $\boldsymbol{x}_{\boldsymbol{i}}=\left(x_{i 1}, \ldots, x_{i p}\right)^{T}$ for each cryptocurrency $i$ where $i=1,2 \ldots, N$, at time t. Among the independent variables some variables are cryptocurrency specific, i.e. they are variables that in one way or another characterise a specific cryptocurrency, such as that cryptocurrency's exchange volume at a certain date. Other variables are not cryptocurrency specific, i.e. they take on the same values regardless of cryptocurrency.

Table 5 offers an overview of the independent variables used to test each hypothesis and their sources. Table 6 presents summary statistics for independent variables that are cryptocurrency specific and Table 7 presents the summary statistics for the other variables. 
Table 5: Overview of independent variables

\begin{tabular}{|c|c|c|c|c|c|}
\hline Hypothesis & Variable [original code] & Code & Source & $\begin{array}{l}\text { Original } \\
\text { frequency }\end{array}$ & Data availability \\
\hline H1 & Number of tokens in circulation & circulating & Coinmetrics & Daily (7-day) & $\begin{array}{l}\text { The data sample stretches back to December } 2013 \text {, but starts } \\
\text { for each cryptocurrency at the time of its introduction }\end{array}$ \\
\hline H2 & Average difficulty & averagedifficulty & Coinmetrics & Daily (7-day) & $\begin{array}{l}\text { The data sample stretches back to December 2013, but starts } \\
\text { for each cryptocurrency at the time of its introduction }\end{array}$ \\
\hline H3 & Exchange volume & exchangevolumeusd & Coinmetrics & Daily (7-day) & $\begin{array}{l}\text { The data sample stretches back to December 2013, but starts } \\
\text { for each cryptocurrency at the time of its introduction }\end{array}$ \\
\hline H4 & Year of implementation & introduction & Coinmetrics & n/a & $\mathrm{n} / \mathrm{a}$ \\
\hline H5 & $\begin{array}{l}\text { Lagged values of log returns of other } \\
\text { cryptocurrencies (lag=1) }\end{array}$ & L.LreturnccC & Coinmetrics & Daily (7-day) & $\begin{array}{l}\text { The data sample stretches back to December 2013, but starts } \\
\text { for each cryptocurrency at the time of its introduction }\end{array}$ \\
\hline H6 & Google searches & google & GoogleTrends & Weekly & Start varies across cryptocurrencies, available to today \\
\hline \multirow[b]{7}{*}{ H7 } & S\&P500 index [GSPC] & SP500 & YahooFinance & Daily (5-day) & To today \\
\hline & NYSE index [NYA] & NYSE & Yahoofinance & Daily (5-day) & To today \\
\hline & AMEX index [XMI] & AMEX & YahooFinance & Daily (5-day) & To today \\
\hline & NASDAQ index [IXIC] & NASDAQ & Yahoofinance & Daily (5-day) & To today \\
\hline & Nikkei225 index [N225] & NIKKEI & Yahoofinance & Daily (5-day) & To today \\
\hline & Shanghai Composite Index (SSE) & SSE & YahooFinance & Daily (5-day) & To today \\
\hline & Oil price & Oil & Quandl/OPEC/ORB & Daily (5-day) & 2001-03-02 to today \\
\hline \multirow[b]{6}{*}{ H8 } & US policy uncertainty index [USEPU] & USEPU & \begin{tabular}{|l} 
policyuncertainty.co \\
$\mathrm{m}$
\end{tabular} & Monthly & 1985 to april 2019 \\
\hline & Europe policy uncertainty index [EEPU] & EEPU & $\begin{array}{l}\text { policyuncertainty.co } \\
\mathrm{m}\end{array}$ & Monthly & 2011 to april 2019 \\
\hline & China policy uncertainty index [CEPU] & CEPU & $\begin{array}{l}\text { policyuncertainty.co } \\
\mathrm{m}\end{array}$ & Monthly & 1995 to april 2019 \\
\hline & CBOE S\&P500 Volatility index [VIX] & VIX & WRDS/CBOE & Daily (5-day) & To 2019-05-31 \\
\hline & CBOE S\&P100 Volatility index [VXO] & vxo & WRDS/CBOE & Daily (5-day) & To 2019-05-31 \\
\hline & CBOE NASDAQ Volatility index [VXN] & VXN & WRDS/CBOE & Daily (5-day) & To 2019-05-31 \\
\hline \multirow[b]{5}{*}{ H9 } & $\begin{array}{l}\text { Exchange rate for People's Republic of China } \\
\text { (Yuan/US\$) }\end{array}$ & exchus & ECB & Daily (5-day) & To today \\
\hline & Exchange rate for Japan (Yen/US\$) & exjpus & ECB & Daily (5-day) & To today \\
\hline & $\begin{array}{l}\text { Exchange rate for United Kingdom Pound } \\
\text { (Pound/US\$) }\end{array}$ & exukus & ECB & Daily (5-day) & To today \\
\hline & $\begin{array}{l}\begin{array}{l}\text { Exchange rate for European Monetary Union } \\
\text { (Euro/US\$) }\end{array} \\
\end{array}$ & exeuus & ECB & Daily (5-day) & To today \\
\hline & Gold price & Gold & Quandl/WGC & Daily (5-day) & 1969-12-29 to today \\
\hline
\end{tabular}

Note: CCC is the abbreviation of three letters used to identify each cryptocurrency, as listed in Table 4. Today means data is updated regularly to give a data availability up until present day, if nothing else is specified the acquired data stretches to 2019-06-08.

\subsubsection{Cryptocurrency specific independent variables}

Table 6 presents the descriptive statistics for the variables that are cryptocurrency-specific, measured before the transformation of the data. The data used for each sub-hypothesis is then described in the following paragraphs.

Table 6: Summary statistics for independent variables that are cryptocurrency-specific

\begin{tabular}{|c|c|c|c|c|c|c|c|c|c|c|c|c|}
\hline \multirow[b]{2}{*}{ Variable: measurement } & \multicolumn{12}{|c|}{ Cryptocurrency } \\
\hline & ADA & $\mathrm{BCH}$ & BTC & EOS & ETC & ETH & LTC & NEO & TRX & $\mathrm{XLM}$ & XMR & XRP \\
\hline Price in USD: mean (st. dev.) & $0.17(0.19)$ & $766.23(633.12)$ & $2547.11(3424.60)$ & $5.61(4.19)$ & $11.21(9.56)$ & $205.26(266.37)$ & $33.90(54.19)$ & $26.66(34.62)$ & $0.032(0.027)$ & $0.075(0.13)$ & $53.67(86.89)$ & $0.78(0.34)$ \\
\hline Price in USD: $\mathrm{min} / \mathrm{max}$ & $\begin{array}{l}.018 / 1.17 \\
0.17 \\
\end{array}$ & $77.37 / 3909$ & $114.45 / 19475.8$ & $0.49 / 21.64$ & $0.604 / 43.86$ & $0.43 / 1397.48$ & $1.15 / 359.13$ & $0.08 / 187.97$ & $0.001 / 0.22$ & $0.001 / 0.89$ & $0.22 / 470.29$ & $0.003 / 3.36$ \\
\hline Return in USD: mean (st. dev.) & $0.00009(0.03)$ & $-0.63(106.28)$ & $1.98(238.48)$ & $0.0049(0.65)$ & $0.004(1.29)$ & $0.10(21.31)$ & $0.029(5.19)$ & $0.10(3.92)$ & $0.0004(0.007)$ & $0.00006(0.015)$ & $0.03(8.28)$ & $0.0001(0.043)$ \\
\hline Return in USD: $\mathrm{min} / \mathrm{max}$ & $-0.17 / 0.31$ & $-639.28 / 1083.97$ & $-2405 / 3536.80$ & $-3.97 / 4.41$ & $-12.11 / 8.25$ & $-231.29 / 154.34$ & $-49.99 / 102.9$ & $-43.24 / 29.54$ & $-0.05 / 0.11$ & $-0.16 / 0.33$ & $-97 / 94.58$ & $-0.92 / 0.76$ \\
\hline Circulating supply: mean (st. dev.) & $\mathrm{n} / \mathrm{a}$ & 652878.5 (346 757.1) & 3472321 (1 682526$)$ & $\mathrm{n} / \mathrm{a}$ & $1.96 \mathrm{e}+07 / 1.12 \mathrm{e}+07$ & $1.84 \mathrm{e}+07(1.01 \mathrm{e}+07)$ & $2.31 e+07(1.09 e+07)$ & n/a & $\mathrm{n} / \mathrm{a}$ & n/a & $1.16 e+07$ ( 4649672$)$ & $n / a$ \\
\hline Circulating supply: $\mathrm{min} / \mathrm{max}$ & $n / a$ & $0 / 1222800$ & $4775 / 5843019$ & $\mathrm{n} / \mathrm{a}$ & $39311.09 / 3.76 \mathrm{e}+07$ & $39311.09 / 3.35 \mathrm{e}+07$ & $29550 / 3.95 \mathrm{e}+07$ & $\mathrm{n} / \mathrm{a}$ & $\mathrm{n} / \mathrm{a}$ & $\mathrm{n} / \mathrm{a}$ & $15562.51 / 1.69 \mathrm{e}+07$ & $n / a$ \\
\hline Average difficulty: mean (st. dev.) & $\mathrm{n} / \mathrm{a}$ & $3.52 \mathrm{e}+11(1.98 \mathrm{e}+11)$ & $1.38 \mathrm{e}+12(2.21 \mathrm{e}+12)$ & $n / a$ & $7.65 e+13(6.08 e+13)$ & $1.30 e+15(1.35 e+15)$ & 1880995 (3 324 578) & n/a & $n / a$ & $n / a$ & $2.16 \mathrm{e}+10(3.10 \mathrm{e}+10)$ & $n / a$ \\
\hline Exchangevolume: mean (st. dev.) & $1.26 \mathrm{e}+08(2.07 \mathrm{e}+08)$ & $6.67 \mathrm{e}+08(8.96 \mathrm{e}+08)$ & $1.90 \mathrm{e}+09(3.34 \mathrm{e}+09)$ & $6.97 \mathrm{e}+08(6.56 \mathrm{e}+08)$ & $1.69 \mathrm{e}+08(2.10 \mathrm{e}+08)$ & $1.08 \mathrm{e}+09(1.42 \mathrm{e}+09)$ & $2.02 \mathrm{e}+08(4.32 \mathrm{e}+08)$ & $1.05 e+08(1.40 e+08)$ & $2.41 \mathrm{e}+08(3.61 \mathrm{e}+08)$ & $3.76 e+07(8.81 e+07)$ & $2.38 \mathrm{e}+07(4.91 \mathrm{e}+07)$ & $2.28 \mathrm{e}+08(6.84 \mathrm{e}+08)$ \\
\hline Exchange volume: $\min / \max$ & $1739460 / 1.71 e+09$ & $85013 / 1.19 \mathrm{e}+10$ & $0 / 2.38 \mathrm{e}+10$ & $4556540 / 4.87 \mathrm{e}+09$ & $267367 / 1.73 e+09$ & $102128 / 9.21 \mathrm{e}+09$ & $0 / 6.96 \mathrm{e}+09$ & $156 / 1.66 \mathrm{e}+09$ & $26475 / 4.09 \mathrm{e}+09$ & $491 / 1.51 \mathrm{e}+09$ & $7900 / 5.44 e+08$ & $0 / 9.11 \mathrm{e}+09$ \\
\hline Date of introduction & $2017-09-24$ & $2017-07-23$ & $2009-01-03$ & $2017-06-20$ & $2015-07-30$ & $2015-07-30$ & 2011-10-07 & 2016-09-09 & $2017-08-28$ & $2014-08-05$ & $2014-04-18$ & 2013-01-02 \\
\hline Google search intensity: mean (st. dev.) & $6.84(10.09)$ & $3.30(7.76)$ & $8.62 / 12.52$ & $72.50(8.82)$ & 13.61 (20.39) & $10.66(16.97)$ & $8.82(12.63)$ & $64.81(13.35)$ & $30.34(9.46)$ & $17.75(7.15)$ & 8.46 (14.04) & $5.04(9.76)$ \\
\hline observations of return & 547 & 617 & 2007 & 639 & 981 & 1333 & 2007 & 934 & 565 & 1700 & 1776 & 2007 \\
\hline
\end{tabular}

\subsubsection{Tokens in circulation}

Number of tokens in circulation can be quantified by looking at the current circulating supply.

CoinMetrics provides a measure of the number of new coins that are brought into existence each day, calculated as the expected number of tokens per block in the blockchain every ten minutes, summed to a daily value of new coins (CoinMetrics, 2018). By summing generated 
coins at the end of each day it is possible to create a value for current circulating supply.

Unfortunately, the CoinMetrics data is only available for the major currencies, Bitcoin, Litecoin, Ethereum, Ethereum Classic, Monero and Bitcoin cash. Thus, this variable should be analysed with caution as a lack of data for minor currencies does not mean that it would not have proved important if the data was available.

\subsubsection{Technical drivers}

In order to test $\mathrm{H}_{2}$ a proxy for technology used is required. Commonly, this is calculated using hash rate. However, the data availability for this variable is only good for the largest cryptocurrencies, such as Bitcoin, and more lacking when it comes to other cryptocurrencies. Therefore, it has not been possible to find a comparable measure of hash rate for a sufficient subset of cryptocurrencies. Instead I use a measure from CoinMetrics, the variable average difficulty. This variable gives a measure for proof of work blockchains of how hard it is to solve the hash function in order to find a new block (CoinMetrics, 2018). Average difficulty is used as a proxy for hash power and is available for Bitcoin, Litecoin, Ethereum, Ethereum Classic, Monero and Bitcoin cash. Thus, this variable should be analysed with caution as a lack of data for other currencies do not signify that it would not have proved important if the data were available.

\subsubsection{Monetary velocity}

Two possible measures of monetary velocity are available in the CoinMetrics dataset that can be used to test $\mathrm{H}_{3}$, namely transaction count and output volume. Transaction count measures the number of transactions happening on the public blockchain per day (CoinMetrics, 2018). A problem with this measurement is that blockchains with low transaction fees typically have more and sometimes smaller transactions. Additionally, some networks, like Bitcoin, can collect several transactions into one which will then underestimate the true value (CoinMetrics, 2018). Thus, this measure is difficult to use for comparison across different cryptocurrencies even if the variable is consistent over time within each cryptocurrency.

A more general approach is offered by the output volume, the total volume of all transaction outputs per day. This is measured as exchange volume in the CoinMetrics dataset which is the dollar value of the volume of each cryptocurrency at major exchanges such as GDAX and Bitfinex (CoinMetrics, 2018). It does not include data on over-the-counter exchanges or other trading platforms, a meaningful proportion of all global exchange, but gives a general image of output volume. The use of both variables, transaction count and output volume, would likely 
result in multicollinearity in the model since number of transactions is one of the variables that contributes to the calculation of output volume. Combining this with the risks associated with the variable for transaction count makes exchange volume the better proxy for monetary velocity and thus this is used for the analysis.

\subsubsection{Network effects - first mover advantage}

The potential first mover advantage resulting from network effects, specified in $\mathrm{H}_{4}$, is measured by comparing cryptocurrencies based on date of implementation across three different time periods, more details in section 5.2. This allows to compare the cryptocurrencies that had been implemented in or before the relevant time period and thereby identify common variables in the resulting models for early or later cryptocurrencies.

As the data sample for each cryptocurrency starts at the time of its introduction, the date of implementation, described in Table 4, is measured as the first date for which data is available on the cryptocurrency in the CoinMetrics dataset. Often, this also corresponds to the date of the first transaction, however not in all cases. A possible explanation for this could be that it takes some time to mine sufficient funds, depending on rules governing supply, to make the first transactions relevant for the cryptocurrency.

\subsubsection{Network effects - reinforcement effect}

The possible impact of network effects, as examined in $\mathrm{H}_{5}$, can be seen through reinforcement or substitution effects in the interaction between cryptocurrencies' returns and how they affect each other. This can be measured by including lagged values of returns for the other cryptocurrencies, using the same calculations for each dependent variable as described in 4.1 and a lag of 1 . A lag of 1 captures the short-term interactions on the cryptocurrency market and can give an adequate image of swift interactions between cryptocurrencies. However, it does not capture long-term movements on the market, and the potential impacts should be interpreted with caution. A deeper analysis into long-term movements on the markets and the inclusion of various lengths of lags for the returns of each cryptocurrency is beyond the scope of this paper but could offer opportunities for future research, for example by evaluating the optimal lag length to be included in the model.

\subsubsection{Speculation}

Several proxies for investor attention are available in order to test $\mathrm{H}_{6}$, such as Wikipedia and Google searches and number of mentions in newspapers. One disadvantage of search history on Wikipedia is that the available information is relatively basic. Thus it is important in the 
early stages of adoption for new users seeking a general understanding, but in the later stages the information becomes less relevant. As a consequence, number of searches will naturally decrease over time. Additionally, a quantification of mentions in newspapers for each cryptocurrency would prove a too extensive analysis to be suitable for the extent of this paper.

Google searches offer a general measure of interest over time, indirectly capturing both Wikipedia searches and mentions in newspaper as these will appear throughout the search history. Thus, for testing $\mathrm{H}_{6}$ investor attention is proxied by worldwide Google searches for the name of the cryptocurrency, available as weekly data from GoogleTrends (2019). The variable gives an index measure of the interest over time, ranging from 0 to a value of 100 for when the interest is at its peak, i.e. the highest number of Google searches (Google Trends, 2019). Thus, a value of 50 indicates that the search term is half as popular as during the peak. A value of 0 indicates that data is missing for the time period.

Google searches might be a rough proxy for some cryptocurrencies with more general names, as their search statistics might include a broader than intended search history. Further I have not taken potential misspellings into account. For a general measure of changes in investor attention this could suffice, particularly given the distinct names of several of the cryptocurrencies. 


\subsubsection{Not cryptocurrency specific independent variables}

Table 7 presents the descriptive statistics for the variables that are not cryptocurrencyspecific, measured before the transformation of the data.

Table 7: Summary statistics for independent variables that are not cryptocurrency-specific

\begin{tabular}{|l|l|r|r|r|r|r|}
\hline Hypothesis & Variable & Obs & Mean & Std. Dev. & Min & Max \\
\hline \multirow{5}{*}{} & SP500 & 2008 & 2254,87 & 331,03 & 1655,45 & 2930,75 \\
\cline { 2 - 7 } & NYSE & 2008 & 11236,00 & 984,60 & 9029,88 & 13637,02 \\
\cline { 2 - 7 } & AMEX & 2008 & 2053,40 & 295,39 & 1592,94 & 2676,69 \\
\cline { 2 - 7 } & NASDAQ & 2008 & 5596,58 & 1200,10 & 3677,78 & 8109,69 \\
\cline { 2 - 7 } & NIKKEI & 2008 & 18740,75 & 2678,55 & 13853,32 & 24270,62 \\
\cline { 2 - 7 } & SSE & 2008 & 2975,65 & 573,78 & 1991,25 & 5166,35 \\
\cline { 2 - 7 } & Oil & 2008 & 63,76 & 22,63 & 22,48 & 110,48 \\
\hline \multirow{5}{*}{ H8 } & USEPU & 2008 & 109,69 & 21,03 & 71,26 & 201,03 \\
\cline { 2 - 7 } & EEPU & 2008 & 203,23 & 60,23 & 111,80 & 433,28 \\
\cline { 2 - 7 } & CEPU & 2008 & 212,57 & 223,37 & 8,02 & 1071,73 \\
\cline { 2 - 7 } & VIX & 2008 & 14,94 & 4,19 & 9,14 & 40,74 \\
\cline { 2 - 7 } & VXO & 2008 & 14,35 & 4,74 & 6,32 & 37,66 \\
\cline { 2 - 7 } & VXN & 2008 & 17,55 & 4,49 & 10,31 & 42,95 \\
\hline \multirow{5}{*}{ H9 } & exeuus & 2008 & 0,85 & 0,07 & 0,72 & 0,96 \\
\cline { 2 - 7 } & exjpus & 2008 & 111,09 & 6,67 & 96,86 & 125,28 \\
\cline { 2 - 7 } & exukus & 2008 & 0,70 & 0,07 & 0,58 & 0,83 \\
\cline { 2 - 7 } & exchus & 2008 & 6,49 & 0,28 & 6,04 & 6,97 \\
\cline { 2 - 7 } & Gold & 2008 & 1244,17 & 68,08 & 1049,40 & 1385,00 \\
\hline
\end{tabular}

\subsubsection{Macroeconomic and financial development}

Changes in macroeconomic and financial development can be quantified by a large variety of variables. In this paper, I have focused on the potential channel offered by stock markets and oil prices. Both are likely influenced by and thereby able to signal changes in macroeconomic and financial development. This makes them relevant proxies in order to test $\mathrm{H}_{7}$.

Changes in regional stock markets is quantified by stock indices. This paper looks at prices for several indices measured on a 5-day week: S\&P500, NYSE, AMEX, NASDAQ, Nikkei225 and Shanghai Composite Index. Changes in oil price are measured by the reference price of the OPEC Crude Oil Basket. This currently includes Saharan Blend (Algeria), Girassol (Angola), Oriente (Ecuador), Iran Heavy (Islamic Republic of Iran), Basra Light (Iraq), Kuwait Export (Kuwait), Es Sider (Libya), Bonny Light (Nigeria), Qatar Marine (Qatar), Arab Light (Saudi Arabia), Murban (UAE) and Merey (Venezuela) (Quandl, 2019b).

\subsubsection{Uncertainty}

In order to test $\mathrm{H}_{8}$ several measures of uncertainty were used, such as the volatility indices (VIX) calculated by CBOE. The VIX index calculations by CBOE are based on the midpoints of bid/ask quotes for options on each index, thereby offering an estimate of expected volatility of the respective equity market (CBOE, 2019). Measures of volatility indices included are 
CBOE S\&P500 VIX, CBOE S\&P100 VIX and CBOE NASDAQ VIX.

Further a measure of political uncertainty for the US (USEPU), Europe (EEPU) and China $(C E P U)$ were included. This is offered by Economic Policy uncertainty calculating a monthly index based on three underlying components: A quantification of newspaper coverage related to policy-related economic uncertainty, a measure of the number of federal tax code provisions set to expire in future years and a third measure that use disagreement among economic forecasters as a proxy for uncertainty (Economic Policy Uncertainty, 2019).

\subsubsection{Hedging}

In order to evaluate the connection to hedging in $\mathrm{H}_{9}$ several measures of classical hedging instruments were selected, such as exchange rate for major currencies and an index price for gold. The exchange rate to USD was included for the People's Republic of China (Yuan, exchus), for Japan (Yen, exjpus), for United Kingdom (Pound, exukus) and for the European Monetary Union (Euro, exeuus). The exchange rates were acquired from the Euro reference rates presented by the ECB and recalculated to dollar-based values for ease of comparison with previous research.

The World Gold Council (WGC) is the market development organization for the gold industry and their 23 members comprise the world's leading gold mining companies (Quandl, 2019a). Thus, the WGC gold price index denominated in USD offers a good measure of developments in gold prices.

\subsection{Stationarity}

A time series displays strict stationarity if the joint distribution of its observations is invariant under time shift (Tsay, 2014). A more common assumption is weak stationarity in which both the mean of the observations and the covariance between observations at different points in time are time invariant (Tsay, 2014). Regressions of interdependent and non-stationary time series may lead to spurious results (Ciaian et al., 2016). Price series are commonly believed to be non-stationary whereas return series, $r_{t}=\ln \left(P_{t}\right)-\ln \left(P_{t-1}\right)$, are stationary (Tsay, 2014).

Stationarity problems in previous research on cryptocurrencies have been dealt with in various ways. Li and Wang (2017) transform their variables by taking the first difference in order to address the non-stationarity in some of their variables. If variables are cointegrated and a first difference of the data is conducted some of this information will be lost. Thus, a first difference 
model can still fail in identification when variables exhibit long-term equilibrium relationships. Li and Wang (2017) address this problem by applying a Vector Error Correction Model (VECM). Kristoufek (2013) tests all standard transformations of the original series, i.e. logarithmic transformation, the first differences and the first logarithmic differences, and his final choice is an application of a Vector Auto Regressive (VAR) and a VECM model. In order to further address co-integration in a mix of stationary and non-stationary time-series, $\mathrm{Li}$ and Wang (2017) use the autoregressive distributed lag (ARDL) model with a bounds test approach in their estimation. Similarly, the ARDL model with different specifications is used by Ciaian et al. (2016) and Bouyoir \& Selmi (2015). As stationarity is a requirement for quantile regression Balcilar et al. (2017) choose to transform Bitcoin index and volume data to stationary returns, obtained as the first differences of the natural logarithmic values of the index expressed in percentage. In order to address stationarity problems, Panagotidis et al. (2018) use logarithmic first differences for most of the variables used.

To investigate stationarity in the data unit root tests were conducted using the Augmented Dickey Fuller test for stationarity with different specifications, both including and excluding a potential trend. The results from the unit root tests were mixed across variables, suggesting that some of the time-series used were not stationary, see Table E2 in Appendix E.

A differencing of variables was conducted to ensure stationarity in the data. Thereby transforming them to return series $r_{t}=\ln \left(P_{t}\right)-\ln \left(P_{t-1}\right)$ similar to Balcilar et al. (2017) and Panagotidis et al. (2018). This transformation yields more comparable measures of growth rates across all variables. Variables that were already stationary before the transformation were still transformed for ease of comparison. Further, there should be no presence of a trend component in a stationary time series, but the results from the unit root tests suggests that there could be some impact of a trend, see Table E2 in Appendix E. As an extra precaution a trend variable, Trend, was included in order to ensure that there are no potential problems with time trends in the data. The variable Trend is continuously defined as 1 for the first observation, 2 for the second, 3 for the third observation and so on.

After the log first difference transformation of the data all variables displayed stationary time series. Table E2 in Appendix E shows the detailed results of the augmented Dickey Fuller test before and after the log first difference transformation of the data. 
Cointegration has not been tested for which could lead to problems such as some loss of information in the data. This could present a point for future research as the presence of cointegration would make a VECM or an ADL a better model choice for investigating the returns of cryptocurrencies. However, for a general overview the LASSO gives comparable results and it has therefore been the choice of this paper.

\subsection{Autocorrelation}

A common problem when looking at time series pricing data is autocorrelation, i.e. serial correlation, the tendency for one observation to be correlated with previous observations (Angrist \& Pischke, 2008). The presence of autocorrelation can be a result of misspecification of variables or omission of relevant explanatory variables (Verbeek, 2017). An OLS regression with these properties could yield inconsistent estimators (Tsay, 2014). Polasik et al. (2015) use both Durbin-Watson and Breusch-Godfrey tests and find no autocorrelation at lag one and no residual serial correlation in their data, therefore choosing to use OLS regression. They further identify that some of their regressions suffer from an endogeneity and simultaneity problem, which they try to solve by using Instrument Variable (IV) regression (Polasik et al., 2015).

When evaluating the available data for each cryptocurrency the Durbin-Watson statistics are close to 2, indicating that first-order residual autocorrelation is not a problem. For more detailed results see Table E1 in Appendix E.

\subsection{Other potential problems}

Balcilar et al. (2017) find that the evidence gained from linear models is not robust to the presence of non-linearity and structural breaks in the data. They suggest quantile regression in order to test for causality not only in means, but also in other quantiles, i.e. upper or lower quantiles (Balcilar et al., 2017). Similarly, Bouri et al. (2017) argue that the use of quantile regression involves a set of regression curves that differ across different quantiles of the conditional distribution of the dependent variable. However, one shortcoming of the quantile regression analysis approach is its inability to capture dependence in its entirety (Bouri et al., 2017). Allowing for differences across quantiles is particularly important if the distribution has fat tails, further it offers the opportunity to divide the market into three states: bear (lower quantiles), normal (median), and bull (upper quantiles) (Balcilar et al., 2017). In their second analysis Bouoyir and Selmi (2017) use Bayesian quantile regression with the similar division of the market into three states, finding that the state of the market matters for the impact of variables. 
Panagotidis et al. (2018) employ the Least Absolute Shrinkage and Selection Operator (LASSO) framework, based on the least-squares approach it considers all potential drivers for changes in the dependent variables but only a subset of the covariates is selected. LASSO is a selection procedure that allows for coefficient shrinkage (even setting some to zero), thereby automating model selection in linear regression (Panagotidis et al., 2018). In order to address structural breaks in the data, Panagotidis et al. (2018) consider both the full sample and a division into three sub-periods. The first period corresponds to an early-adoption phase with relatively low volatility (July $24^{\text {th }} 2010$ to October $1^{\text {st }} 2013$ ), the second period begins with the boom in late 2013 and the Mt.Gox's bankruptcy and corresponds to the crash and gradual recovery of Bitcoin (October $2^{\text {nd }} 2013$ to January $3^{\text {rd }}$ 2017), whereas the last period corresponds to the recent alleged bubble (January $4^{\text {th }} 2017$ to June $23^{\text {rd }}$ 2017) (Panagotidis et al., 2018).

A deeper investigation into quantile regression lies beyond the scope of this paper given the number of cryptocurrencies to be evaluated. Structural breaks on the other hand could have an impact on the data used and will be considered, more details in the method section 5.2 structural stability.

\section{Method}

Panagotidis et al. (2018) describes the advantage of using LASSO regression as it allows to consider all potential drivers but only a subset of the variables is selected. Using the LASSOapproach for testing the hypotheses exploits the variance-bias trade-off, the LASSO reduces the complexity for the model, through shrinking or setting some coefficients to zero, thereby decreasing the variance of the prediction. However, in doing so it allows for a potential bias to be introduced into the model. This bias risk making the magnitudes of the estimated effects less reliable and one should be cautious when interpreting them. The aim of this paper is to evaluate homogeneity among cryptocurrencies, for this purpose the selection, direction of impact and relative importance of potential drivers of returns for each cryptocurrency gives enough information in order to evaluate the hypotheses. Thus, for the remaining of this paper the analysis will not discuss the exact magnitudes of the results found but rather focus on the bigger picture of homogeneity among cryptocurrencies.

In order to prove or disprove homogeneity the exact magnitude might not be of the highest importance, but rather that the selection and relative importance of the potential drivers of returns are the same for all cryptocurrencies. That is, if we take the resulting models from a 
LASSO-approach and rank the strength of the impact of the coefficients, a similar ranking for all cryptocurrencies should be found if they are homogenous. For example, if Bitcoin is seen as a representative currency with $\mathrm{A}, \mathrm{B}$ and $\mathrm{C}$ as its strongest drivers of returns then this should be the same for all other cryptocurrencies. For example, results showing that Ethereum have B, D and $\mathrm{F}$ as its strongest drivers of returns would disprove the homogeneity assumption. For a visualisation see Figure 3.

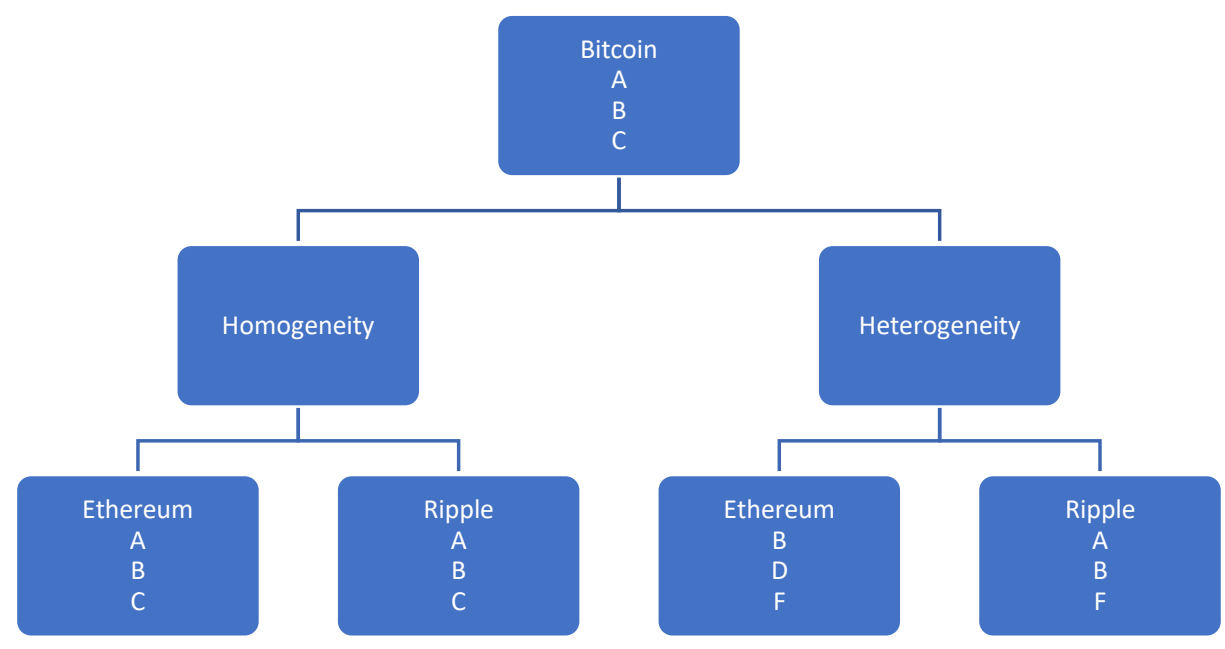

Figure 3: Example of expected results with homogeneity as compared to heterogeneity

\subsection{LASSO}

The Least Absolute Shrinkage and Selection operator (LASSO) was first introduced by Robert Tibshirani (1996). The LASSO methodology is a penalized least squares technique which by using a constraint allows some coefficients to shrink towards or be exactly 0 (Tibshirani, 1996). One underlying assumption for the LASSO regressions is sparsity, that the underlying true model of the data is sparse and that we seek a sparse solution in which many of the estimators, $\hat{\beta}$, are equal to zero (Gauraha, 2018). This assumption makes the LASSO able to handle highdimensional models where the number of regressors are large, possibly even exceeding the number of observations. Taken together, this makes the LASSO able of producing both interpreTable models similar to subset selection and sTable estimates similar to Ridge regression (Tibshirani, 1996).

Similar to the OLS methodology, the LASSO minimizes the residual sum of squares. However, the LASSO tries to address two of the main reasons for why OLS regression often yield nonsatisfactory estimates. The first is prediction accuracy: OLS estimates often have low bias but large variance and prediction accuracy can sometimes be improved by shrinking towards, or setting some coefficients equal to 0 (Tibshirani, 1996). The shrinking towards zero removes the 
variability in a model associated with many small coefficients, thus the right amount of shrinkage can provide a more sTable solution but offers a risk of introducing some bias to the model (Gauraha, 2018). The second is interpretation: Even if we have a large number of predictors we might want to determine a smaller subset that exhibits the strongest effects, thereby representing a more interpreTable model (Tibshirani, 1996).

Suppose that we have data $\left(\boldsymbol{x}_{\boldsymbol{i}}, y_{i}\right), i=1,2 \ldots, N$, where $\boldsymbol{x}_{\boldsymbol{i}}=\left(x_{i 1}, \ldots, x_{i p}\right)^{T}$ are the predictor variables and $y_{i}$ are the responses. We assume that the $y_{i}$ s are conditionally independent given the $x_{i j}$ s and that the $x_{i j} \mathrm{~s}$ are standardized so that $\sum_{i} x_{i j} / N=0, \sum_{i} x_{i j}^{2} / N=1$.

With $\hat{\beta}=\left(\hat{\beta}_{1}, \ldots, \hat{\beta}_{p}\right)^{T}$, the LASSO estimator is defined as:

$$
\hat{\beta}^{\text {lasso }}=\arg \min \left\{\sum_{i=1}^{N}\left(y_{i}-\beta_{0}-\sum_{j=1}^{P} \beta_{j} x_{i j}\right)^{2}\right\} \text { subject to } \sum_{j=1}^{P}\left|\beta_{j}\right| \leq t
$$

Here $t \geq 0$ is a tuning parameter that controls the amount of shrinkage that is applied to the estimates. For all $\mathrm{t}$, the solution to $\beta_{0}$, the intercept, becomes $\hat{\beta}_{0}=\bar{y}$. Without loss of generality we can thus assume that $\bar{y}=0$ and hence omit $\beta_{0}$.

Let $\hat{\beta}_{j}^{0}$ be the full least squares estimates and let $t_{0}=\Sigma\left|\hat{\beta}_{j}^{0}\right|$. This constraint makes the solutions non-linear in $y_{i}$, and a quadratic programming algorithm is used to compute them (Hastie, Tibshirani \& Friedman, 2001). Values of $t<t_{0}$ will cause shrinkage of solutions towards 0 , and some coefficients may be exactly equal to 0 . This allows for model selection. If $t \geq t_{0}$ then the LASSO estimator is the same as the OLS estimator, which in turn allows for sTable estimation of the coefficients.

The LASSO problem can equivalently be written in Lagrangian form as:

$$
\hat{\beta}^{\text {lasso }}=\arg \min \left\{\frac{1}{2} \sum_{i=1}^{N}\left(y_{i}-\sum_{j=1}^{P} \beta_{j} x_{i j}\right)^{2}+\lambda\left(\sum_{j=1}^{P}\left|\beta_{j}\right|\right)\right\}
$$

And estimated through cvlasso from the Stata package lassopack (Ahrens, Hansen \& Schaffer, 2019). Following Panagotidis et al. (2018) and common practice lambda, $\lambda$, is allowed to vary and a value for it is chosen through cross-validation. Cross-validation divides the data repeatedly into training and validation data where the model is fit to the training data and the validation data is used to check the prediction error (Hastie et al., 2001; Ahrens et al., 2019). 
This in turn allows identification of which values of $\lambda$ that optimize predictive performance by minimising the estimated mean-squared prediction error (Hastie et al., 2001; Ahrens et al., 2019).

A rolling cross validation methodology with 10 folds was implemented to make the optimal use of the time-series structure in the dataset (Hyndman \& Athanasopoulos, 2018). The 1-step ahead cross-validation splits the data into 10 groups of approximately equal size, $\mathrm{k}=1 \ldots 10$. The first fold is treated as the validation dataset and the remaining K-1 parts constitute the training dataset. Rolling cross validation considers the time-series structure in that the training window is iteratively extended (moved forward) by one step. This methodology helps finding the optimal value of $\lambda$, the value that minimizes the estimated mean-squared prediction error.

The cvalsso statapack implements a range of lambdas to be tested where each lambda must be greater than 0 (Ahrens et al., 2019). The default maximum value is equal to $2 * \max \left(\mathbf{X}^{\prime} \mathbf{y}\right)$, where $\mathbf{X}$ is the pre-standardized regressor matrix and $\mathbf{y}$ is the vector of the response variable (Ahrens et al., 2019). The default minimum value must be between 0 and 1 and is calculated via a ratio of minimum to maximum lambda, usually 1/1000 (Ahrens et al., 2019).

A high lambda yields a tightly fit model in which several coefficients are shrunken to exactly zero, whereas a low lambda allows more coefficients to remain in the optimal model. The minimisation of mean-squared prediction error by calculating the optimal value of lambda can only be solved if a local or global minimum falls within the range of the tested lambdas as it does in A) but not in C) in Figure 4. Further, if the quadratic function is strictly decreasing there might not exist a unique optimal value of lambda given the current data and specifications used, see B) in Figure 4 for an example.

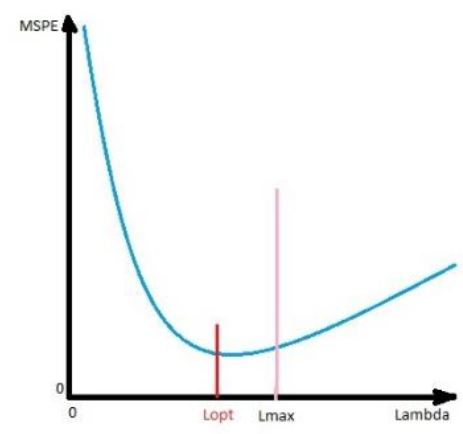

A) An optimal value of lambda exists within the lambda range

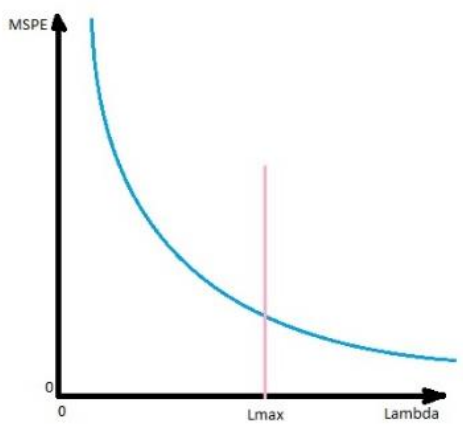

B) No optimal value of lamda is defined within the lamda range

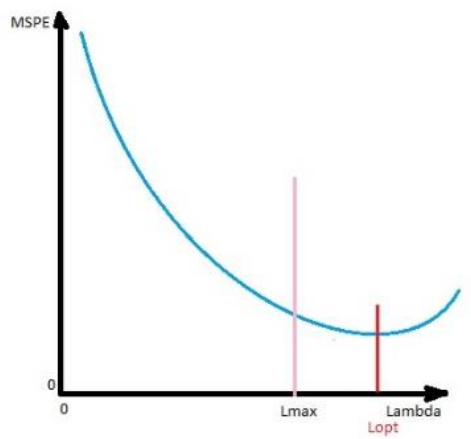

C) An optimal value of lambda exists outside the lambda range

Figure 4: Illustration of different solutions for optimal lambda 
Running the tests for a range of lambda values will only yield the results for values within the range, thus there is no way to distinguish between case B) and C) if the results of the mathematical operations are inconclusive. However, the max value of lambda implemented tends to be sufficiently high to shrink all coefficients to zero. If the optimal value of lambda is at the end of the lambda range this could then suggest that the current data or the variables used are not enough to determine what the optimal model should be. The selected variables are likely not the main drivers of returns for the cryptocurrency in the investigated time period.

\subsection{Structural stability}

The first part of the LASSO-analysis was conducted using all available data. No restrictions regarding dates were implemented which gives a good overview but can make the results sensitive to variables with little available data, such as returns for cryptocurrencies with a relatively recent date of implementation. The shrinking procedure of the LASSO operator should make sure that variables with too little data are excluded if they introduce more variation than what they are able to explain in the model, thus having their coefficients shrunken to zero. Results are shown in Table 8 in section 6.1. Out of the cryptocurrencies examined only XLM could not define a unique value for lambda in this part of the analysis and is hence omitted from the following comparisons.

Structural breaks are unexpected changes over time in the parameters used in a time-series model, for example coefficients in the model can be different before and after a major change in macro-economic policy (Verbeek, 2017). The presence of structural breaks in the data can make the resulting model unreliable as what is relevant in one time period might not be relevant in the next. The great variation and difficulty in interpreting the results from the first part of the analysis, as seen in Table 8 in section 6.1, suggests that a further division of the data is needed in order to extract comparable models. It is possible that the market for cryptocurrencies have undergone large changes over time (for example with respect to attention in media or acceptance as medium of exchange or investment) resulting in structural breaks in the data.

The current information on development and specific events in the cryptocurrency market is highly focused on Bitcoin, and there is little or limited information in the current research on market development for other cryptocurrencies. As the aim of this paper is to test homogeneity among cryptocurrencies it will consequently also evaluate the representativeness of Bitcoin. If homogeneity among cryptocurrencies is true, then a division into time periods based on the different phases the Bitcoin market have been through should not pose a problem and it 
becomes possible to use the available information. Following Panagotidis et al (2018), a division into three different time periods were implemented in order to take structural breaks in the data into account, for a visualisation see Figure 5:

1. Crash (2013-10-02 to 2017-01-03). This time period omits the first early market of Bitcoin and other cryptocurrencies where the volatility of prices and existing userbase were relatively low. Thereby, the analysis starts from the Bitcoin boom in early 2013, the subsequent crash and gradual recovery of Bitcoin up until the beginning of 2017 (Panagotidis et al., 2018). Cryptocurrencies that had not yet been implemented in this time period are omitted from the analysis and as explanatory variables $(\mathrm{EOS}, \mathrm{BCH}$, TRX and ADA).

2. Recent (2017-01-04 to 2017-06-23). This time period encompasses the more recent market and alleged bubble of Bitcoin and follows the work of Panagotidis et al. (2018) for ease of comparison. Cryptocurrencies that had not yet been implemented in this time period are omitted from the analysis and as explanatory variables $(\mathrm{EOS}, \mathrm{BCH}, \mathrm{TRX}$ and ADA).

3. Current (2017-06-23 to 2019-04-01). This time period continues beyond the scope of Panagotidis et al. (2018) into current day. It is meant to capture the current development where cryptocurrencies have become more accepted and increasingly implemented into the traditional economy. It also encompasses a period with higher volatility as compared to the previous time periods.

\section{Bitcoin Price (BTC)}

$\$ 10,641.17 \triangle 0.630379 \%$

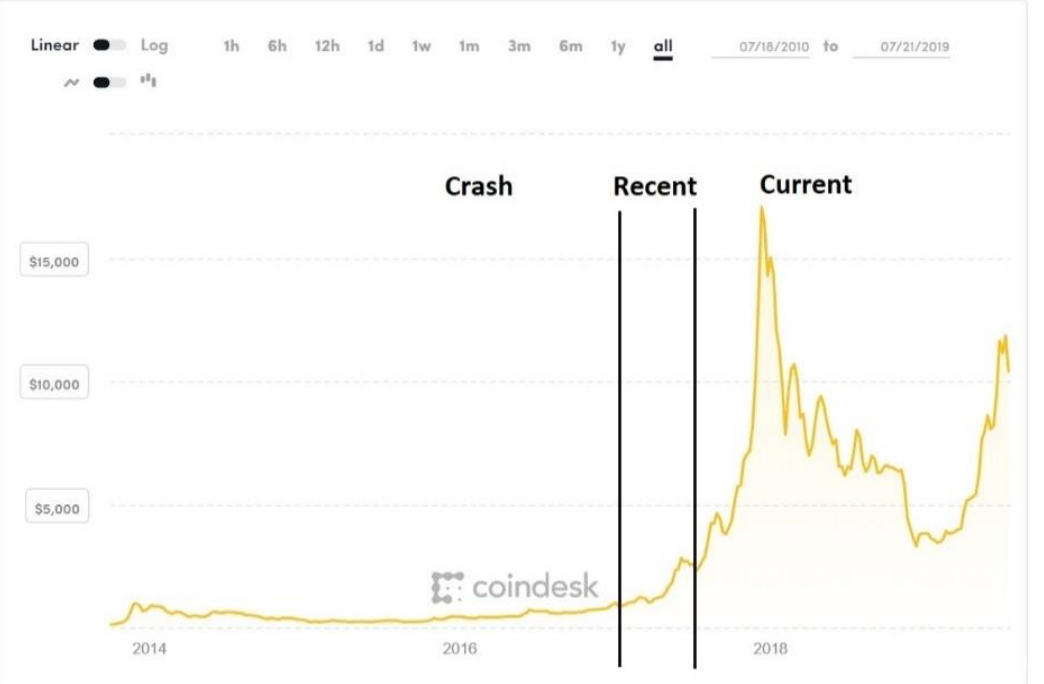

Figure 5: Illustration of development of Bitcoin price index in time periods used

Source: https://www.coindesk.com/price/Bitcoin (2019-07-21), edited by including time periods 
The LASSO-analysis have been conducted for each subcategory of time separately. For a full overview of the results when divided into subcategories of time see Table 9 in Section 6.1.

\subsection{The impact of cryptocurrencies' design choices}

Generally, all cryptocurrencies display large variation in what variables are relevant in explaining returns when comparing across time. The large variation could suggest that different explanatory variables are important at different stages of implementation for a cryptocurrency. It is also possible that cryptocurrencies with similarities in design choices, as described by the questions in Table 1 in Section 2.2, go through similar stages of implementation. If that is the case, the importance of design choices for a cryptocurrency could be seen when comparing across design choices for each time period. Thus, the subsequent analysis is divided into four parts based on the questions in Table 1:

1. How are tokens created?

2. How are tokens distributed and transactions validated?

3. What is the target market for the token?

4. What is the token being used for?

The results from the LASSO-regression have been divided by category for each of the questions above. Any common factors found in the division by categories when evaluating the hypothesis could help explain and further the understanding of what makes some variables relevant in explaining the returns of cryptocurrencies. If there exists variation in the impact of some variables but the impacts appear to be common over categories, then it is possible that cryptocurrencies sharing these characteristics display some level of homogeneity that could help explain the impact.

\subsection{Endogeneity}

The models tested in this paper could suffer from endogeneity and simultaneity problems, the factors assumed to be driving returns could in fact be influenced by the returns of a cryptocurrency. This is not a likely problem for the variables that are not cryptocurrency specific, i.e. take on the same value regardless of cryptocurrency, as they are mainly broad indices measured by and influenced by several global factors. The current size of the cryptocurrency market and its limited usability as compared to traditional asset classes makes it unlikely that changes in returns for one individual cryptocurrency could cause changes in for example volatility indices or indices connected to the stock markets. However, it could be more 
problematic for some of the cryptocurrency specific variables. The supply variables that are defined for each cryptocurrency at their time of implementation cannot be adjusted to accommodate changes in returns, hence circulating supply, average difficulty and year of implementation are not endogenous. Similarly, values for returns cannot be influenced by future returns which makes the variables with lagged returns of other cryptocurrencies exogenous.

Exchange volume and Google searches are both influenced by the interest in and perceived usefulness of a cryptocurrency. Exchange volume could be endogenous if higher returns of a cryptocurrency led to more intensive trade with larger transactions. Investor attention proxied by Google searches could be endogenous if higher returns of a cryptocurrency increased the interest and demand for that cryptocurrency. Both drivers of returns are extensively used throughout the current literature, suggesting a praxis in which they are considered relevant in examining the broader image. Thereby they are included in this paper, thus accepting some risk of endogeneity in the models. There are some alternative ways to deal with these potential endogeneity problems. These are generally beyond the scope of this paper and offer interesting areas of future research. One example could be to implement instrument variables, similar to the choice of Polasik et al. (2015), or to gain a deeper understanding of how interest in and perceived usefulness of a cryptocurrency changes and what influences it, e.g. by studying behavioural economics connected to cryptocurrencies.

\subsection{Limitations}

Using the LASSO-approach for testing the hypotheses exploits the variance-bias-tradeoff. It reduces the complexity for the model, but in doing so it allows for a potential bias to be introduced into the model. For the testing of the hypotheses in this paper the LASSO provides a number of models with comparable ranking and selection of the drivers of returns that are relevant for each cryptocurrency. As long as the bias does not change the relative order of the relevant drivers of returns it should only lead to a slightly higher caution in interpreting the magnitude of the effects. Given that log-transformations of the first difference of some variables reduce autocorrelation and provides stationarity of the time-series used should also help reduce the potential bias of the estimates. Adding lagged values of previous returns provides some short-term autoregressive elements to the models, however an extensive investigation into the most appropriate number of lags to be used is beyond the scope of this paper. Further, the great number of variables already included in the model makes it hard to include several lagged 
values of all cryptocurrencies. Thus, for simplicity and in order to capture short term movements on the cryptocurrency market a lag of 1 is selected. As a consequence, these variables are likely better at capturing speculation such as day-to-day trading, rather than longterm investments

Structural breaks in the data could be tackled by dividing the data into subsamples based on specific events occurring for Bitcoin, similar to the three sub-periods used by Panagotidis et al. (2018). If Bitcoin is a representative currency, i.e. homogeneity holds, then choosing the subperiods based on events related to Bitcoin should not impact the relative importance of drivers of returns for other cryptocurrencies. By contrast, if Bitcoin is not a representative cryptocurrency then this division might not be suitable for all cryptocurrencies evaluated, introducing a risk of unreliable models.

Another way to tackle developments in the market is to divide the market and compare across quantiles of returns, for example comparing across bull, bear and normal modes as in the paper by Bouoiyour \& Selmi (2017). The investigation of differences across quantiles of the returns for cryptocurrencies is beyond the scope of this paper as it would simply result in too extensive calculations, however it holds good potential for future research and in-depth analysis.

Historically, cryptocurrencies have had a strong connection with illegal activities and a large share of the initial trading of Bitcoin was related to illegal activities, such as drug dealing and trafficking. The ECB identifies the main risks a government faces related to cryptocurrencies as means of payment arise in cases when cryptocurrencies are used for money laundering, tax evasion or the financing of terrorism $(\mathrm{ECB}, 2015)$. The black market is increasingly adopting the opportunities for e-commerce and digital payments through cryptocurrencies (Foley, Karlsen \& Putniņš, 2018). Approximately one-quarter (25\%) of all users and one-half of Bitcoin transactions (44\%) are associated with illegal activity (Foley, Karlsen \& Putniņš, 2018). These proportions are decreasing, possibly due to increased regulatory attention, overall more investors or the emergence of alternative cryptocurrencies with higher level of anonymity (Foley, Karlsen \& Putniņš, 2018). However, it is possible that a significant component of cryptocurrencies' value as payment systems comes from facilitating illegal activities (Foley, Karlsen \& Putniņš, 2018). If illegal activity, such as money laundering, is facilitated by the use of cryptocurrencies, it would contribute to driving the demand for a cryptocurrency. Out of the risks described by the ECB (2015), it is hard to find relevant metrics, especially related to tax evasion and financing of terrorism. The lack of objective data in the matter places the question 
of the potential role of facilitating illegal activity and its implications for returns of cryptocurrencies beyond the scope of this paper.

\section{Results}

This section presents the results of the LASSO-analysis, first in its entirety in Table 8 and 9, and later divided into four parts based on the initial questions from Table 1, Tables of results are available in Appendix A through D:

1. How are tokens created? (Appendix A)

2. How are tokens distributed and transactions validated? (Appendix B)

3. What is the target market for the token? (Appendix C)

4. What is the token being used for? (Appendix D)

The data is divided into three time periods, as described in Section 5.2, and each time period is looked at separately for each question in order to identify common patterns among cryptocurrencies. The aim is to present the findings in a more nuanced way and to avoid the influence of looking for particular results in order to confirm or reject the chosen hypotheses. A complete analysis for each hypotheses is conducted under Section 7. Analysis.

\subsection{Overview}

The first part of the analysis was conducted with no restrictions on the data related to time frames, resulting in Table 1 with cryptocurrencies sorted by date of implementation.

As can be seen in Table 8, the optimal values for lambda vary greatly across cryptocurrencies and only XLM did not have a defined lambda. BTC, LTC, XMR and ADA have lambdas that are high enough to result in an optimal model where none of the chosen variables should be included to explain their returns. Out of those BTC, LTC and XMR are among the earliest implemented cryptocurrencies which could suggest that they have reached a new level of development in which the current theoretical setting is unable to explain their returns.

Other cryptocurrencies offer more information. XRP, ETH, NEO, EOS and TRX have optimal values for lambdas ranging from 2.77 to 7.7 resulting in more comparable models. For 4 out of 5 among those cryptocurrencies (XRP, ETH, NEO and EOS), Google searches have a positive 
impact on returns. For 3 out of 5 (ETH, NEO and EOS) the returns for XRP have a negative impact on returns. For the cryptocurrencies in which the optimal lambda results in a model with only one relevant variable (ETC and $\mathrm{BCH}$ ), indicate that average difficulty has a positive impact on their returns.

In order to take structural breaks into account and to generate more comparable models the division into subcategories of time (Crash, Recent and Current) were conducted. For an overview of the results see Table 9. Out of the time periods and cryptocurrencies tested only XLM did not have a defined lambda for the current time period and is henceforth omitted from the analysis.

Table 8: Overview LASSO-analysis with no restrictions on time

\begin{tabular}{|c|c|c|c|c|c|c|c|c|c|c|c|c|c|}
\hline \multicolumn{2}{|c|}{ Cryptocurrency } & BTC & LTC & XRP & XMR & XLM & ETC & ETH & NEO & EOS & $\mathrm{BCH}$ & TRX & ADA \\
\hline \multicolumn{2}{|c|}{ Optimal lambda } & 5.853 & 10.683 & 3.62 & 8.39 & $\overline{\text { not def. }}$ & 12.12 & 3.374 & 2.364 & 2.77 & 9.613 & 7.7 & 9.379 \\
\hline $\mathrm{H} 1$ & circulating & & & $n / a$ & & $\mathrm{n} / \mathrm{a}$ & & & $\mathrm{n} / \mathrm{a}$ & $n / a$ & & $\mathrm{n} / \mathrm{a}$ & $\mathrm{n} / \mathrm{a}$ \\
\hline $\mathrm{H2}$ & averagedifficulty & & & $n / a$ & & $n / a$ & 0.2264 & 0.0054 & $n / a$ & $n / a$ & 0.0730 & $\mathrm{n} / \mathrm{a}$ & $\mathrm{n} / \mathrm{a}$ \\
\hline \multirow[t]{12}{*}{$\mathrm{H3}$} & exchangevolumeusd & & & & & & & & 0.0090 & & & & \\
\hline & L.LreturnADA & & & -0.0455 & & & & & & & & & $n / a$ \\
\hline & L.LreturnBCH & & & & & & & & -0.0013 & & $n / a$ & & \\
\hline & L.LreturnBTC & $n / a$ & & -0.0168 & & & & & & & & & \\
\hline & L.LreturnEOS & & & & & & & & & $n / a$ & & & \\
\hline & \begin{tabular}{|l|} 
L.LreturnETC \\
\end{tabular} & & & -0.0450 & & & $n / a$ & & & & & -0.0667 & \\
\hline & L.LreturnETH & & & & & & & $n / a$ & & & & & \\
\hline & L.LreturnLTC & & $n / a$ & & & & & & & 0.0530 & & & \\
\hline & L.LreturnNEO & & & & & & & & $n / a$ & & & & \\
\hline & L.LreturnTRX & & & 0.1056 & & & & & 0.0279 & & & $n / a$ & \\
\hline & L.LreturnXLM & & & & & $n / a$ & & & 0.0065 & & & 0.0778 & \\
\hline & L.LreturnXMR & & & & $n / a$ & & & & -0.0492 & & & & \\
\hline H5 & L.LreturnXRP & & & $n / a$ & & & & -0.0086 & -0.0563 & \begin{tabular}{|l|}
-0.0399 \\
\end{tabular} & & & \\
\hline \multirow[t]{7}{*}{ H6 } & google & & & 0.0290 & & & & 0.0824 & 0.5983 & 0.6986 & & & \\
\hline & SP500 & & & & & & & & & & & & \\
\hline & NYSE & & & & & & & & & & & & \\
\hline & AMEX & & & & & & & & 0.3361 & 0.0949 & & & \\
\hline & NASDAQ & & & & & & & & & & & & \\
\hline & NIKKEI & & & & & & & & & & & & \\
\hline & SSE & & & & & & & & & -0.1345 & & & \\
\hline \multirow[t]{6}{*}{$\mathrm{H7}$} & Oil & & & & & & & & & & & & \\
\hline & USEPU & & & & & & & & & & & & \\
\hline & EEPU & & & 0.4699 & & & & & & & & & \\
\hline & CEPU & & & & & & & & -0.0743 & -0.1462 & & & \\
\hline & $\mathrm{VIX}$ & & & -0.0381 & & & & & & & & & \\
\hline & $\mathrm{VXO}$ & & & & & & & & & & & & \\
\hline \multirow[t]{5}{*}{ H8 } & VXN & & & & & & & & \begin{tabular}{|l|}
0.0436 \\
\end{tabular} & & & & \\
\hline & exchus & & & & & & & & & & & & \\
\hline & exjpus & & & & & & & & & & & & \\
\hline & exukus & & & & & & & & & & & & \\
\hline & exeuus & & & & & & & & -0.5074 & -0.5168 & & & \\
\hline \multirow[t]{3}{*}{ H9 } & Gold & & & & & & & & & & & & \\
\hline & Trend & & & & & & & & & & & & \\
\hline & Constant & -0.0001 & 0.0002 & 0.0004 & -0.0009 & - & -0.0018 & \begin{tabular}{|l|}
-0.00127 \\
\end{tabular} & $\mid-0.0021$ & 0.0035 & $\mid-0.0018$ & 0.0042 & 0.0021 \\
\hline
\end{tabular}




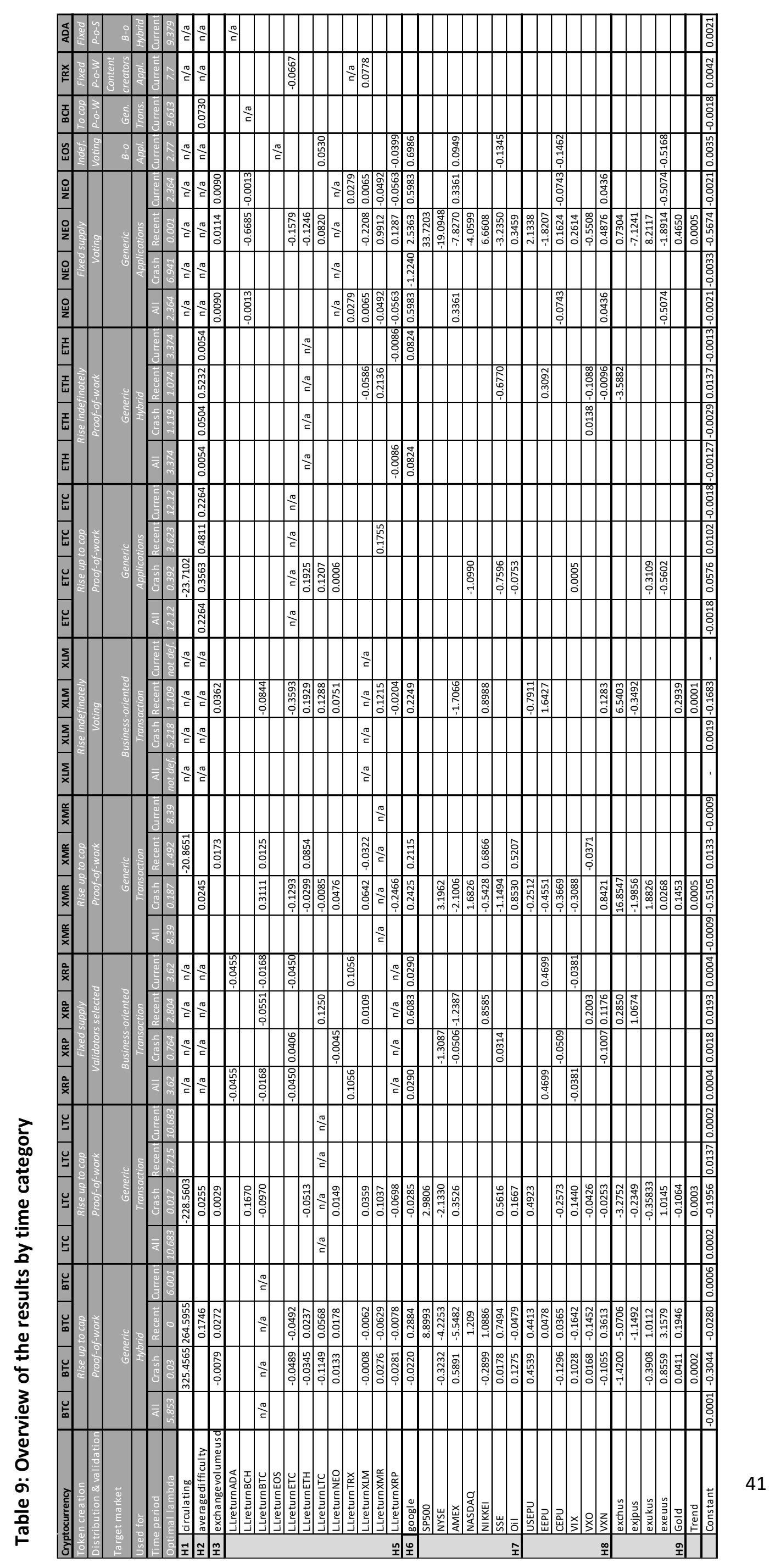


Generally, all cryptocurrencies display large variation in what variables are relevant in explaining returns when comparing across time. As can be seen in Table 9 a few cryptocurrencies have variables that remain relevant throughout all three time periods, such as average difficulty for ETC and ETH, and Google searches for NEO.

\subsection{Design choices}

Table 9 offers an overview of the results but in order to understand why some variables are relevant in explaining returns in some time periods and others are not we need a little bit more information. By comparing the results from Table 9 divided by design choices it becomes possible to identify potential common factors. As the information compared is the same across all design choices the resulting Tables are presented in Appendix A through D and the comparisons across time periods presented below according to each question.

\subsubsection{How are tokens created?}

The Tables of results are presented in Appendix A. Looking at Table A1, in the first time period there are no common variables in the group of cryptocurrencies with fixed supply or where supply rises indefinitely. Generally, these categories also use higher optimal lambdas resulting in less variables used in their optimal models. In contrast the cryptocurrencies with supply that rise up to cap have lower lambdas, suggesting that the theoretical framework used here is more adapted to explaining variation in this category. For the category 'rise up to cap' several variables are common across all models in Table A1, the return of ETH, LTC and NEO, variations in SSE and oil prices, VIX indices and currency exchange rates towards the UK and the EU. However, the impacts of these variables are not consistent, for some cryptocurrencies the impact on returns is positive whereas for others the impact is negative. Further, the large magnitude of the impact of circulating supply is remarkable, with a positive impact for BTC and a negative impact for LTC and ETC.

When looking at the Recent time period in Table A2 it is easier to find common variables in the categories for both fixed supply and supply that rise indefinitely. For cryptocurrencies with fixed supply the impact on returns is positive for both NEO and XRP from variables such as return of LTC, Google searches, NIKKEI and VXN index and exchange rate towards China. The impact on returns is negative for both NEO and XRP for the AMEX index. For cryptocurrencies where supply rises indefinitely, lagged return of XRP and increases in CEPU 
index have a positive impact on both ETH and XLM. The results in Table A2 become less clear when looking at cryptocurrencies with a supply that rises up to cap. In the current time period, the optimal lambda for LTC results in a model where none of the variables are relevant in explaining returns. Even if excluding LTC and comparing the other three cryptocurrencies (BTC, ETC and XMR) there are no common variables for the current time periods.

In the third time period, looking at the current market in Table A3, there are no variables that are common for all cryptocurrencies in any of the groups. For several of the cryptocurrencies with a supply that rises up to cap the optimal lambda results in a model with none of the current variables used in the final model (BTC, LTC and XMR). For the remaining cryptocurrencies average difficulty remains important with a positive impact on returns for ETC and BCH. For this category the high lambdas in the third category stands in strong contrast to the lower lambdas in the crash and recent periods, possibly suggesting a new turn of development that makes the current theoretical framework less suitable to explain current returns.

\subsubsection{How are tokens distributed and transactions validated?}

The resulting Tables are presented in Appendix B. In the first time period, crash as seen in Table B1, it is clear that the variables are relevant in explaining a lot of the variation in returns for cryptocurrencies using proof-of-work, whereas the resulting models for cryptocurrencies using voting suggests that the theoretical framework is less adapted to explaining changes in their returns. Since there is only one cryptocurrency using selected validators, it is not possible to compare across common explanatory variables. For cryptocurrencies using proof-of-work, only the return of ETH is relevant and common across all cryptocurrencies in Table B1. Further, there are several variables that are relevant for 4 out of 5 cryptocurrencies. Average difficulty and the return of NEO both have a positive impact on returns for LTC, ETC, ETH and XMR. The impact goes in different directions, some positive some negative, when looking at increases in SSE, oil and VIX indices, currency exchange rate towards UK and EU.

In the second time period, as seen in Table B2, the variables become more relevant in explaining a lot of the variation in returns for cryptocurrencies using voting, and less relevant for cryptocurrencies using proof-of-work. There are no longer any variables that are common for all cryptocurrencies using proof-of-work. Average difficulty remains important with a positive impact on 3 out of 5 cryptocurrencies (BTC, ETC and ETH). However, in Table B2, there are several common variables for cryptocurrencies using voting. The impact on returns for both NEO and XLM is positive for exchange volume, lagged returns of LTC and XMR, increases in 
the NIKKEI and VXN indices, the exchange rate towards China and increases in gold price. The impact on returns for both NEO and XLM is negative for the lagged return of ETC, increases in the AMEX index and the exchange rate towards Japan. Other common variables with different directions of impact on returns are lagged returns of ETH and XRP, and increases in the USEPU and EEPU indices.

In the third time period, seen in Table B3, the variables have lost most of their relevance in explaining variation in returns for cryptocurrencies using proof-of-work, leaving no variables in the final model that are common across all cryptocurrencies in this category. Interestingly, average difficulty is still among the most relevant variables for cryptocurrencies using proofof-work, able to explain some of the variation in returns for ETC, ETH, BCH and TRX. Since there is only one cryptocurrency using either selected validators or proof-of-stake it is not possible to compare across common explanatory variables. For cryptocurrencies using voting, XLM does not have a uniquely defined lambda for the Current time period, thus comparison in this category is only made between NEO and EOS from Table B3. The impact on returns for both NEO and EOS is positive for increases in Google searches and the NASDAQ index. The impact is negative for lagged returns of XRP, increases in the VIX index and exchange rate towards the EU.

\subsubsection{What is the target market for the token?}

The Tables of results are presented in Appendix C. In the first time period, seen in Table C1, it is hard to find common variables that have an impact on returns for both business-oriented and generic cryptocurrencies. The business-oriented cryptocurrencies, XLM and XRP, offer too little information for comparison. For the cryptocurrencies with a more generic target market it is easier to find common variables, although none remain important for all 6 cryptocurrencies. Average difficulty has a significant positive impact on returns for 4 out of 5 possible cryptocurrencies (LTC, ETC, ETH and XMR). Similarly, the lagged return of NEO has a positive impact on 4 out of 5 cryptocurrencies (BTC, LTC, ETC and XMR).

In the second time period, seen in Table C2, the image becomes a bit clearer for businessoriented cryptocurrencies with several common variables impacting returns. The lagged returns of Bitcoin and increases in the AMEX index both have a negative impact on returns for XLM and XRP. The lagged return of LTC, Google searches, increases in the NIKKEI and VXN indices and exchange rate towards China all have a positive impact on returns for both XLM 
and XRP. For the cryptocurrencies with a more generic target market there are no variables that are important for all 6 cryptocurrencies in Table C2. The lagged returns of XLM and increases in the VXO index both have a negative impact on 4 out of 6 cryptocurrencies (BTC, ETH, XMR and NEO) whereas the lagged returns of XMR have a more mixed impact.

When looking at the third time period in Table C3 it is difficult to find common variables in all three categories of target markets, generic, business-oriented and directed towards content creators online. Further, since there is only one cryptocurrency focusing on content creators it is not possible to make comparisons across common explanatory variables in that category. Among the cryptocurrencies with a generic target market, the average difficulty stands out in that it has a positive impact on 3 out of 6 possible cryptocurrencies. Further the lagged returns of XRP have a negative impact on both ETH and NEO, whereas the impact on those cryptocurrencies are negative for Google searches. Among the business-oriented cryptocurrencies only Google searches show a positive impact on at least 2 out of 4 possible cryptocurrencies (EOS and XRP).

\subsubsection{What is the token being used for?}

The Tables of results are presented in Appendix D. Looking at Table D1, in the first time period there are no variables that are common for all cryptocurrencies used for transaction purposes. However, average difficulty has a positive impact on both cryptocurrencies with available data (LTC and XMR) and there are several variables that are common for 3 out of 4 cryptocurrencies, although with different direction of impact. For cryptocurrencies used mainly for applications there are no common variables influencing returns of NEO and ETC in Table D1. However, in the hybrid cases, the VXO index has a positive impact on returns for both BTC and ETH.

When looking at the second time period in Table D2 there are still no variables that are common for all cryptocurrencies used for transaction purposes. The lagged returns of BTC have a positive impact on returns for XMR but a negative impact on returns for XRP and XLM. Increases in Google searches have a positive impact on returns for 3 out of 4 cryptocurrencies (XRP, XMR and XLM). For cryptocurrencies used mainly for applications, the lagged returns of XMR have a positive impact on the returns for both NEO and ETC. Among the hybrid cryptocurrencies, average difficulty has a positive impact on returns for both BTC and ETH whereas the impact of other common variables is more mixed. 
In the third time, as seen in Table D3, no variables remain common in the categories of cryptocurrencies being used for transaction or hybrid purposes. Among the cryptocurrencies used for application purposes there are some that are common for at least 2 out of 4 possible cryptocurrencies. The lagged return of XLM has a positive impact on returns for both NEO and TRX. The lagged returns of XMR have a negative impact on returns for both NEO and EOS, whereas the impact of Google searches and increases in the NASDAQ index are positive. Both increases in the CEPU index and exchange rate towards the EU have a negative impact on returns for NEO and EOS.

\section{Analysis}

This section discusses the results along the sub-hypotheses $\mathrm{H}_{1}$ to $\mathrm{H}_{9}$. It demonstrates that only $\mathrm{H}_{2}$ could not be rejected, since the choice of technical drivers and hash rate have a similar impact on the cryptocurrencies for which data is available. This makes it possible to reject the overarching null hypothesis, and thus, confirming the alternative hypothesis: Cryptocurrencies are non-homogenous, i.e. heterogenous, with respect to drivers of returns. Consequently, Bitcoin should not be seen as a representative cryptocurrency.

\subsection{Tokens in circulation}

Variations in number of tokens in circulation creates differences in determinants of returns among cryptocurrencies. Those differences appear to, at least partly, be possible to explain in that number of tokens in circulation is only relevant for cryptocurrencies that share characteristics such as having a supply that rise up to cap and utilising a proof-of-work design. Bitcoin stands out in that circulating supply has a positive impact on its returns, whereas the impact is negative for the other cryptocurrencies. This suggests that Bitcoin should not be seen as a representative cryptocurrency with respect to the impact of circulating supply.

When looking at Table 8, LASSO-analysis with no restrictions implemented, there is no difference between the cryptocurrencies. Differences in number of tokens in circulation is not relevant in explaining the determinants of the returns for cryptocurrencies where data is available. This is also true when looking at the current time period in Table 9, but slightly different in the earlier time periods. During the crash time period, the circulating supply is relevant for explaining returns of BTC, LTC and ETC, whereas in the recent time period it is only relevant for BTC and XMR. The effect is positive for BTC, a higher supply leads to a higher return in both time periods, but negative for LTC, ETC and XMR. 
BTC, LTC, ETC and XMR all have supply that rise up to cap which, when looking at Table A1 and A2 in Appendix A, seems to offer some explanation for the effect. Like the findings of $\mathrm{Li}$ and Wang (2017), the supply for those cryptocurrencies only has a significant effect in the earlier markets, possibly suggesting the impact of some large event in the market of the cryptocurrency or a new stage in the development and adaption phases of a cryptocurrency. In contrast to Ciaian et al. (2016), the effect here is positive for Bitcoin, possibly due to differences in measurements or choice of models. That the effect is negative for the other cryptocurrencies with supply that rise up to cap could suggest that they follow a similar development curve as Bitcoin, in which increases in supply lead to lower prices, ceteris paribus, in the crash and recent market. Since the time-periods chosen exclude the early introduction phase of Bitcoin it is possible that Bitcoin has already passed some kind of turning point, establishing enough demand that its strict rules on increasing supply can no longer fulfil the demand on the market. If the demand is high enough then the predictable increases in supply of Bitcoin will still not be enough to decrease prices. It is also possible that as the supply of Bitcoin increases so does the number of possible trading partners, thus in turn increasing demand further and leading to higher prices.

Unfortunately, it is hard to draw any conclusions as there is only one other cryptocurrency with information about token creation available for which circulating supply is not relevant, ETH. The situation is the same when looking at distribution as a potential source of explanation in Table B1 and B2 in Appendix B, except for ETH. All cryptocurrencies for which circulating supply is relevant share the characteristic that distribution and validation of transactions are based on proof-of-work. However, the information on circulating supply is further only available for this category of cryptocurrencies. It is not possible to know what the results would have been if the information would have been available for all cryptocurrencies.

Taken together, this variation in impact of number of tokens in circulation on the cryptocurrencies for which data is available makes it possible to reject $\mathrm{H}_{1}$ : Variations in number of tokens in circulation do not create any differences in determinants of returns for cryptocurrencies.

\subsection{Technical drivers}

$\mathrm{H}_{2}$ states that variations in hash rate between cryptocurrencies do not create any differences in determinants of returns. The proxy used to evaluate this, the variable average difficulty is only 
available for some of the cryptocurrencies. Among those, there are strong similarities in effect of average difficulty on returns. As can be seen in Table 9, the effect is especially strong in the first time period, crash, and continuous throughout all three time periods for some cryptocurrencies. The similarities in sign and occurrence over time of the effect suggests that at least some of the cryptocurrencies are similarly impacted, improvements in technical drivers such as hash rate contribute to higher returns. However, due to the lack of data, the effects found might only reflect the characteristics of the cryptocurrencies with the information available, such as proof-of-work protocols and generic target market.

When looking at Table 8, the hash rate is relevant in explaining returns for ETC, ETH and LTC. The effect is positive, and it remains positive through all three time periods for ETH and ETC as seen in Table 9. It is also relevant for LTC and XMR in the crash market and BTC in the recent market, all with positive impact on returns. This supports the arguments of Dwyer (2015) that the choice of technical drivers and characteristics could contribute to a positive value for a cryptocurrency and is also in line with the results of Bouoiyour and Selmi (2015).

Taking the different time periods into account the data on average difficulty offers too little information in order to motivate what is common across the cryptocurrencies. For example, the results in Appendices B and C offer no comparative cryptocurrencies in other categories, the information on average difficulty is only available for cryptocurrencies using proof-of-work and having a generic target market. This could suggest that proof-of-work and generic target markets are common factors for cryptocurrencies where a higher hash rate leads to higher returns for some cryptocurrencies. However, given the lack of information it is not possible to conclude if the results would be similar for the other categories if data was available.

The similarities in sign and occurrence over time of the effect for the cryptocurrencies for which data is available suggests that $\mathrm{H}_{2}$ should not be rejected: Variations in hash rate do not create any differences in determinants of returns for cryptocurrencies.

\subsection{Monetary velocity}

The similarities in impact in recent and current time periods as seen in Table 9 could suggest some homogeneity among the cryptocurrencies in how they are impacted by changes in monetary velocity. However, the large difference between the number of cryptocurrencies where the impact is relevant in explaining returns and the number of cryptocurrencies where it 
is not indicates that the effect of exchange volume is not homogenous across all cryptocurrencies. In addition, in the first period the effect on returns for BTC moves in opposite direction.

Looking at Table 8, where no restrictions are implemented, exchange volume is only relevant in explaining returns of NEO. This suggests that increases in the total volume of all transactions outputs with NEO have a positive impact on the returns of NEO. Looking at the more detailed image with different time periods in Table 9 the impact is negative for BTC in the crash period but positive and relevant for the other cryptocurrencies in the crash period (LTC), in the recent period (XMR, XLM, BTC, NEO) and in the current period (NEO). It is not possible to distinguish any common characteristics when looking at design choices of the cryptocurrencies in Appendices A through D, rather the impact of monetary velocity seems to affect some cryptocurrencies in every possible category. This in turn could suggest that the common factor is something that is not captured by this division into types of design choices, but that there could still exist similarities across cryptocurrencies.

One possible explanation for the negative effect on BTC returns in the crash period, in Table 9, can be found in the study by Bouoiyour \& Selmi (2017), where, similarly, the impact on BTC is negative for the bear state quantiles. As this study does not include a way to measure the state of the market it is not possible to evaluate its potential impact. The positive impact of exchange volume on the other cryptocurrencies corresponds to the findings of Polasik et al. (2015), who concluded that monthly change in number of Bitcoin transactions have a significant positive impact on Bitcoin returns. For the cryptocurrencies where the exchange volume is not relevant, the results show similar outcomes to those of Ciaian et al. (2016), who find no significant impact on their tested measure, days destroyed.

Given the large variation in how monetary velocity is measured in previous studies it becomes hard to draw conclusions, it is possible that the choice of measurement affects which cryptocurrencies display relevant impacts on returns. As the variable used for this study, exchange volume, does not include data on over-the-counter exchanges and trading platforms it is possible that the results would be more informative if it was possible to use an overall measure of all transaction outputs. The strong focus on exchanges with the variable tested could imply that we see an effect for cryptocurrencies where trading on exchanges is a large share of the volume but that cryptocurrencies with alternative trading routes are not captured to the same extent. 
Taken together, this large difference between the number of cryptocurrencies where the impact is relevant in explaining returns and the number of cryptocurrencies where it is not, combined with the distinct impact on Bitcoin returns, makes it possible to reject $\mathrm{H}_{3}$ : Variations in monetary velocity do not create any differences in determinants of returns of cryptocurrencies.

\subsection{Network effects - first mover advantage}

The division of the data into several time periods allows for some comparisons related to date of implementation. Comparison over time shows that variations in date of implementation appears to create some differences in returns for the cryptocurrencies. Particularly when considering the changes over time in number of relevant variables or looking at the impact of average difficulty as a competitive advantage. However, it is hard to isolate the effects and to fully distinguish it from the impact of e.g. proof-of-work protocol as design choice. Choosing another method that more clearly takes the time aspect into account could offer more detailed results and clearer insights into the impact of network effects and the role of date of implementation.

When looking at the first time period in Table 9, older cryptocurrencies tend to have more variables that are relevant in explaining their returns. The older cryptocurrencies that share the characteristics of a proof-of-work protocol (BTC, LTC and XMR) also have several variables in common, as can be seen in Table B1 in Appendix B. The more recent cryptocurrencies have less variables relevant and common in explaining their returns in the crash period (XLM, ETC, ETH and NEO).

In the recent time period in Table 9, the image becomes more mixed and it is hard to distinguish any particular common factors related to date of implementation. When looking at the third time period it is clear that the variables are slightly more relevant in explaining returns for later cryptocurrencies, as compared to the older ones where none of the selected variables should be included in the final model (BTC, LTC, XMR and XLM). For the cryptocurrencies with data on average difficulty, the variable is relevant for the more recent cryptocurrencies (ETC, ETH and $\mathrm{BCH}$ ) but not for the older ones (BTC, LTC and XMR). The impact is positive, possibly suggesting that more recent cryptocurrencies use better hash rates to gain an edge in competition against the incumbent cryptocurrencies. This could be a sign of the presence of network effects in the cryptocurrency market. Further Google searches offer a mixed image, more relevant for the recent cryptocurrencies (ETH, NEO and EOS) but not for the newest ones (BCH, TRX and 
ADA).

The movement from more relevant variables to fewer for the older cryptocurrencies, as seen in Table 9, could imply that the current economic framework was very suitable for explaining returns for the older cryptocurrencies during the crash time period, with several relevant variables, but not as well fitted in the current time period. This supports the view of Corbet et al. (2018), results that were obtained in 2016 are probably not the same if obtained in 2018, and thus it is necessary to continue conducting research. The framework offers some relevance for more recent cryptocurrencies but not to the same extent as for the older cryptocurrencies in the crash time period. Given the large difference in numbers of variables that are relevant in explaining returns and how they vary across older and younger cryptocurrencies in different time periods, it is possible that we are looking at the cryptocurrencies at different stages of their development. It is also possible that the current division into different time periods, as it is based on BTC, is not as suitable for the other cryptocurrencies leading to unreliable models.

Taken together, variations in date of implementation appears to create some differences in returns for the cryptocurrencies, thus making it possible to reject $\mathrm{H}_{4}$ : Variations in date of implementation do not create any differences in determinants of returns of cryptocurrencies.

\subsection{Network effects - reinforcement effect}

Taken together, the large variations in relevance over time and whether the impact is positive or negative suggests that the cryptocurrency market is highly integrated with large interactions and interdependencies among cryptocurrencies in the short term. However, there is no clear cut "winner-takes-all" race across all three time periods distinguishable from Table 9 and the possible substitution effects change over time, suggesting movements on a very shifting market. This could be a consequence of the short-term time horizon chosen by only evaluating the interactions among cryptocurrencies with a lag of 1 and its possible that a more extensive study would yield more conclusive results.

The impact of lagged values of returns for competing cryptocurrencies move in different directions in different time periods for almost all cryptocurrencies. As can be seen in Table 9, in the first time period, the returns of BTC is negatively impacted by all relevant cryptocurrencies which suggests a leading position in the "winner-takes-all" race. Increases in returns of other cryptocurrencies have a negative impact on return of BTC, possibly due to a substitution effect towards alternative cryptocurrency investments. However, this changes in 
the recent and current time period in Table 9. In the recent time period, the impact on BTC returns from increases in other cryptocurrencies is mixed, with both positive and negative impact, and in the third time period none of the other cryptocurrencies' returns remain relevant. One possible explanation could be if in the third time period BTC was well enough established to no longer be threatened by the other cryptocurrencies. For all other cryptocurrencies the results in Table 9 are mixed in all time periods, both with positive and negative impact. This suggests that a strong interaction between cryptocurrencies exists and that it has an impact on returns. However, it might not be as clear cut as a "winner-takes-all" race and it might rather be the results of movements on a very liquid market where the substitution effects changes depending on the current relative popularity of the cryptocurrencies.

It is hard to find common factors that could help explain which cryptocurrencies display an impact and which ones do not. For the crash period, Table A1 and B1, in Appendices A and B respectively, suggests that cryptocurrencies with a supply that rise up to cap or with a proof-ofwork protocol are more sensitive to the returns of other cryptocurrencies. However, these differences disappear in the later time periods. For cryptocurrencies where the impact of the returns of other cryptocurrencies is not relevant a common factor appears to be the choice of how tokens are created. Looking at Table A1 in Appendix A, in the crash period cryptocurrencies with a supply that rise indefinitely (ETH, XLM) are not impacted by the returns of other cryptocurrencies. By contrast, in Table A3 cryptocurrencies with a supply that rise up to cap (BTC, LTC, ETC, XMR and BCH) are the ones not impacted by returns of other cryptocurrencies.

The impact of lagged returns of other cryptocurrencies varies over time across all cryptocurrencies in Table 9, thus making it possible to reject $\mathrm{H}_{5}$ : Lagged values of returns for competing cryptocurrencies do not create any differences in determinants of returns of cryptocurrencies. There is no distinguishable move towards either a "winner-takes-all" race or a substitution effect.

\subsection{Speculation}

As can be seen in Table 9, generally the impact on returns of investor attention, proxied by Google searches, is positive for many of the cryptocurrencies. However, there exists some interesting deviations in the first time period and there are several cryptocurrencies for which Google searches have no impact. The lack of effect for some cryptocurrencies could be a result 
of the choice of variable, Google searches is a very rough proxy for cryptocurrencies with more general names, and thus the full effect for all cryptocurrencies might not have been captured.

When looking at the results with no limitations implemented in Table 8, investor attention measured by Google searches is relevant in explaining returns with a positive impact for four cryptocurrencies (XRP, ETH, NEO and EOS). When dividing the sample into time periods in Table 9 investor attention generally remains an important variable but it is not possible to find common design choices suggesting an explanation for why. The impact of investor attention is generally positive, increases in Google searches have a positive impact on returns for a cryptocurrency, which is in line with previous findings (Kristoufek, 2013; Panagotidis et al., 2018; Bouyoir and Selmi, 2015; Bouyoir \& Selmi, 2017; Li and Wang, 2017; Ciaian et al., 2016; Polasik et al., 2015). However, some cryptocurrencies in the crash time period in Table 9 deviate from this (NEO, BTC and LTC). All three of them have a generic target market, as can be seen in Table C1 in Appendix C, and increases in Google searches in the crash period have a negative impact on their returns. One possible explanation for this could be if the increased investor attention were taken as a sign of a bubble in the cryptocurrency's price which in turn made the generic investors turn away from the cryptocurrency, thus decreasing returns. Further investigation into this potential explanation is beyond the scope of this paper but it could offer interesting insights into the cryptocurrency market.

Overall the results indicate that changes in investor attention impacts cryptocurrencies differently, especially when looking at different time periods, and thus it is possible to reject $\mathrm{H}_{6}$ : Variations in level of speculation, proxied by investor attention, do not create any differences in determinants of returns of cryptocurrencies.

\subsection{Macroeconomic and financial development}

The large variation and differences in effects across cryptocurrencies suggests that variations in macro-financial development creates differences in determinants of returns of cryptocurrencies, both over time and for each cryptocurrency.

Looking at the unrestricted results in Table 8, only NEO and EOS are impacted by changes in macroeconomic and financial developments. For both NEO and EOS, increases in the AMEX index have a positive impact on returns. This suggests that investors in those cryptocurrencies could be influenced by changes in the American stock market. Both cryptocurrencies are being used for applications, as can be seen in Appendix D. One potential explanation could be if 
companies working with the applications related to cryptocurrencies were to a large extent based in the US. When the stock market captured by the AMEX index improves this signals a better situation for those companies which in turn spills over to increased demand for their products and in the extension the demand increases for the cryptocurrencies which in turn increase their returns. EOS is further negatively impacted by increases in the SSE index. One potential explanation for this could be if EOS was extensively used as an alternative investment to traditional Chinese stock. Then as the Chinese market improves, the investors are not seeking out alternative investments to the same extent thereby decreasing the demand for the cryptocurrencies and reducing returns.

The impact of stock and oil indices varies across all cryptocurrencies over time, as can be seen in Table 9. In the third time period the impact seems less common, as compared to in the earlier time periods. This could suggest that early investors used cryptocurrencies as a complementary investment to their traditional stocks and that later we see a movement towards less interaction between the markets. It is possible that the cryptocurrency market has established itself as a market on its own and that spill-over effects across markets could be less problematic today as compared to earlier time periods.

This large variation and differences in how macro-financial development influence returns of cryptocurrencies, makes it possible to reject $\mathrm{H}_{7}$ : Variations in macroeconomic and financial development do not create any differences in determinants of returns of cryptocurrencies.

\subsection{Uncertainty}

When looking at the unrestricted results in Table 8 some effects of regional uncertainty have a positive impact on returns (EEPU on XRP, VXN on NEO) whereas others have a negative impact on returns (CEPU on NEO and EOS, and VIX on XRP). The image does not become clearer cut when looking at subcategories of time periods in Table 9. In the crash period, when uncertainty is relevant, the effect is mixed for all cryptocurrencies, except for CEPU where the impact is strictly negative. One potential explanation for this could be if a relatively large share of those investments came from China. If uncertainty arise in China, then investments in cryptocurrencies become less attractive for Chinese investors and they might prefer to move their investments into safer assets, thus decreasing demand and consequent returns for cryptocurrencies. 
Generally, in the first time period, as seen in Table 9, the impact of uncertainty on returns were relevant for the most cryptocurrencies and this seems to have decreased over time to a quite low relevance in the current time period. In addition, during the crash time period the cryptocurrencies with a supply that rise up to cap or that could be defined by a proof-of-work protocol all had at least one uncertainty index that was relevant in explaining their returns, as seen in Table A1 and B1 in Appendices A and B respectively. This effect becomes much more mixed in the recent time period and then completely disappears in the current time period.

The impacts of different measures of uncertainty varies between positive and negative, not displaying any clear patterns over time for any of the cryptocurrencies in Table 9. Further there is no uncertainty index that have an either all positive or all negative impact on returns for all the cryptocurrencies for which it is relevant. Taken together, this suggests large differences among cryptocurrencies in how their returns are impacted by global and regional uncertainty and thus it is possible to reject $\mathrm{H}_{8}$ : Variations in global and regional uncertainty do not create any differences in determinants of returns of cryptocurrencies.

\subsection{Hedging}

Hedging could be a motivation in using cryptocurrencies, but if so the cryptocurrencies are not used to hedge for the same uncertainty and thus the resulting impact is not homogenous across cryptocurrencies.

Looking at Table 8, where no restrictions are implemented, it is only the Euro to Dollar currency exchange rate that has a relevant impact on returns for cryptocurrencies. The impact is negative for NEO and EOS, both cryptocurrencies have an established voting system for distribution and validation of transactions and are extensively used for applications. Especially in the current time period the impact of the traditional hedging variables is only relevant for cryptocurrencies used in applications, see Table D3 in Appendix D. If increases in traditional hedges signals uncertainty and lead to increased demand for hedging opportunities, then the negative impact of cryptocurrencies used for applications could signal that they are connected to risky investments and thus demand for these decreases in times of uncertainty. The choice of a voting system for validation could further signal a stand away from traditional market forces. The variables chosen are very broad measures and it is possible that they capture something other than the potential to use cryptocurrencies for hedging. 
Overall, the impact of exchange rates and the gold price index were more relevant in the earlier time periods, as can be seen in Table 9. During the crash time period the cryptocurrencies with a supply that rise up to cap or that could be defined by a proof-of-work protocol all had at least one traditional hedging variable that was relevant in explaining their returns, see Table A1 or B1 in Appendices A and B respectively. This effect becomes much more mixed in the recent time period and then completely disappears in the current time period. Further, there is no traditional hedge variable that has an either all positive or all negative impact on returns for all the cryptocurrencies for which it is relevant.

The large differences in impact on returns for different cryptocurrencies and what potential uncertainties the cryptocurrencies are used to hedge for makes it possible to reject $\mathrm{H}_{9}$ : Variations in financial development for assets traditionally used for hedging do not create any differences in determinants of returns of cryptocurrencies. 


\section{Conclusion}

Standard economic goods are priced by the interaction of demand and supply and by extension classic economic theory implies that the price of a good reflects all current available and relevant information. Cryptocurrencies are fiat currencies where the rules governing supply are set out at the initial launch of the cryptocurrency, whereas demand is influenced by its value in future exchanges. This makes market characteristics, such as the pricing mechanisms, returns and potential drivers of returns interesting subjects for study as they contain a lot of information, both information on the cryptocurrency itself and on factors that influence its value and the cryptocurrency market.

The aim of the study was to examine if cryptocurrencies are homogeneous. The question of homogeneity among cryptocurrencies is answered by looking deeper into the pricing mechanisms of the cryptocurrency market in a LASSO analysis. The drivers of returns that have been identified for Bitcoin in a contemporary theoretical framework were applied to a sample of 12 cryptocurrencies. Thereby offering comparison between cryptocurrencies, further analysing over time and across design choices of cryptocurrencies.

The findings show that cryptocurrencies are not homogenous, i.e. they are heterogeneous with respect to drivers of returns. One potential exception is identified in that the choice of technical drivers and hash rate have a similar impact on the cryptocurrencies for which data is available. Several prior studies have noted the importance of hash rate (Dwyer, 2015; Bouyoir and Selmi, 2017) and the positive impact of the variable average difficulty is in line with their results. Increases in technical drivers contribute to higher returns, especially for some of the more recent cryptocurrencies, which seems to use it to gain an edge in the competition against the incumbent cryptocurrencies in a market with strong positive network effects.

Sections 7.4 and 7.5 show the presence of network effects and that short-term interactions between cryptocurrencies seem to matter on the cryptocurrency market, which is consistent with the work of Gandal and Halaburda (2014). Cryptocurrencies appear to undergo stages of development, and there could exist similarities across cryptocurrencies in different stages that could be further explored. It is also possible that the cryptocurrency market has developed past a simple hedge for uncertainty in traditional assets and has now established itself as a market on its own, thereby making interaction with for example stock markets less relevant for its pricing mechanism. From a regulatory perspective this could suggest a decrease in the systemic risk through potential direct or indirect exposure. However, more research is needed to confirm 
the developments in the cryptocurrency market. An evaluation of the market interactions with a greater emphasis on the long-term perspective, i.e. by including more lags on returns of other cryptocurrencies would help further the understanding of the potential implications of network effects in the cryptocurrency market.

This paper has also shown that Bitcoin displays several distinguishing traits, thus making it unlikely that it could be seen as a representative cryptocurrency. For example, the effect on Bitcoin return stands out as compared to the other cryptocurrencies, for variables measuring tokens in circulation and monetary velocity. When looking at the relation to other cryptocurrencies, Bitcoin appears to have taken a lead in a "winner-takes-all-race" in the earlier time periods, but this lead is later replaced by substitution effects and shifting interactions between all cryptocurrencies, suggesting high integration on the cryptocurrency market.

The results in sections 7.7 and 7.8 indicate less spill-over effects between cryptocurrency and traditional markets in current time as opposed to earlier time periods. However, the results from section 7.4 and 7.5 indicate high short-term interconnectedness on the market. From a regulatory perspective, the decreased risk of contagion across markets is positive but the high interconnectedness could offer cause for concern. One risk could be if cryptocurrencies are perceived to have high similarities in risk exposure. This combined with a high integration of the cryptocurrency market could lead to potential problems spreading, from one cryptocurrency to another, on the cryptocurrency market. This in turn could potentially harm relatively unprotected investors and increase the risks for bubbles in the cryptocurrency market.

Overall the findings show that cryptocurrencies are heterogenous and that the different design choices related to demand and supply appear to matter and can in several cases offer some explanations for the effects of variables. Cryptocurrencies with a supply that rise up to cap appear to be more sensitive to circulating supply. In the earlier time period, cryptocurrencies with a supply that rise up to cap were more sensitive to the returns of other cryptocurrencies, whereas cryptocurrencies with a supply that rise indefinitely were not impacted. In the more recent time period, this image is reversed and only cryptocurrencies with either fixed supply or supply that rise indefinitely were impacted by other cryptocurrencies' returns. Cryptocurrencies that are used for applications appear to be slightly more sensitive to stock market development and traditional hedging measures. 
One drawback of this study has been the limitations in available data, for example a lack of data for circulating supply and average difficulty, and a rough proxy for investor attention in Google searches. Another drawback was the lack of cryptocurrencies in some categories which has made comparison difficult, for example in Appendix B, only one cryptocurrency falls under the validators selected or proof-of-stake categories respectively. The impact of the variables used is often mixed, both in the relevance and the direction of impact. The limited availability of data, both on cryptocurrency specific variables and on variation in design choices, often makes it hard to evaluate the results. A larger sample of cryptocurrencies, or better underlying data for each cryptocurrency could help mitigate some of these drawbacks and shed more light on the cryptocurrency market. In particular, it could confirm or reject that some of the effects found can truly be attributed to proof-of-work protocol as a design choice.

The findings may also be somewhat limited by the choice of model and the exact magnitudes of the effects should be interpreted with caution. The LASSO-approach when used for testing hypotheses exploits the variance-bias trade-off, thereby yielding more comparable models but possibly introducing a bias. Several transformations of the data are conducted to decrease the risks associated with this potential bias. This method offers comparative results across cryptocurrencies and sufficient information to reject the overarching null hypothesis for this study. However, in order to extend the knowledge on cryptocurrencies and the cryptocurrency market future research might wish to look at specific cryptocurrencies more in detail, possibly also taking cointegration into account by applying VECM or ADL models. In those cases, the resulting optimal models based on causality in means from the LASSO-methodology could be too restrictive. A non-linear analysis allowing for differences across each cryptocurrency's quantiles of the conditional distribution of the dependent variable, would likely yield more information at the cost of a more extensive analysis. This would be a good future focus of research in order to deepen the understanding of a subsample of cryptocurrencies with specific characteristics, such as for example proof-of-work-protocols.

One of the issues that emerges from these findings is the need for continuous research on cryptocurrencies. In the earlier time period (2013-10-02 to 2017-01-03), the current theoretical framework was relevant in explaining returns for cryptocurrencies sharing characteristics such as proof-of-work protocols for transaction validation and supply that rise up to cap. In contrast, in the more current time period (2017-06-23 to 2019-04-01), the theoretical framework was less fitted for explaining their returns. Overall, the impact of non-cryptocurrency specific factors 
has decreased over time for all cryptocurrencies, suggesting that the current theoretical framework might not be as applicable today as it was in the early years of implementation. This is in line with the arguments of Corbet et al. (2018): results obtained in earlier time periods are not the same when obtained in later time periods and should thereby not be considered equivalent. This also indicates that continuous research on cryptocurrencies, both for Bitcoin and other cryptocurrencies, is needed.

Cryptocurrencies are to their nature global, but their investors are often individuals with a more local angle of incidence. In-depth analysis of behavioural economics could help clarify how the cryptocurrency market works and decrease the risks associated with endogeneity in the current models. In particular, if it could offer a deeper understanding of for example user activity, the role of speculation and investor motivation and the perceived substitutability between cryptocurrencies. The aim should be to gain a deeper understanding of how individuals' interest in and perceived usefulness of a cryptocurrency changes and what influences it. Further, the potential connection to illegal activity as identified by for example Foley, Karlsen \& Putniņš, (2018) is a matter that needs to be considered when determining possible regulation of cryptocurrencies and future studies on the subject is therefore recommended.

The findings in this paper show that cryptocurrencies are heterogenous, thus the strong Bitcoin focus could make the existing models and current economic framework less relevant in explaining how the cryptocurrency market works. There is a risk for misinterpretation and misdirected regulation if this heterogeneity among cryptocurrencies is not considered. More research is needed, both empirical and theoretical, especially into the details of what makes cryptocurrencies similar and how they interact. 


\section{References}

Ahrens, A., Hansen, C.B., \& Schaffer, M.E. (2019). lassopack: Model selection and prediction with regularized regression in Stata. arXiv:1901.05397.

Ali, R., Barrdear, J., Clews, R., \& Southgate, J. (2014). The economics of digital currencies. Bank of England Quarterly Bulletin, Q3.

Angrist, J. D., \& Pischke, J. S. (2008). Mostly harmless econometrics: An empiricist's companion. Princeton university press.

Balcilar, M., Bouri, E., Gupta, R., \& Roubaud, D. (2017). Can volume predict Bitcoin returns and volatility? A quantiles-based approach. Economic Modelling, 64, 74-81.

Baur, D. G., Dimpfl, T., \& Kuck, K. (2018). Bitcoin, gold and the US dollar-A replication and extension. Finance Research Letters, 25, 103-110.

Bianchi, D. (2018). Cryptocurrencies as an asset class? An empirical assessment. An Empirical Assessment (June 6, 2018). WBS Finance Group Research Paper.

Bouri, E., Gupta, R., Tiwari, A. K., \& Roubaud, D. (2017). Does Bitcoin hedge global uncertainty? Evidence from wavelet-based quantile-in-quantile regressions. Finance Research Letters, 23, 87-95.

Bouoiyour, J., \& Selmi, R. (2015). What does Bitcoin look like?. Annals of Economics and Finance, 16(2), 449-492.

Bouoiyour, J., \& Selmi, R. (2017). The Bitcoin price formation: Beyond the fundamental sources. arXiv preprint arXiv:1707.01284.

Burnie, A. (2018). Exploring the interconnectedness of cryptocurrencies using correlation networks. arXiv preprint arXiv:1806.06632.

CBOE (2019). White Paper CBOE Volatility Index. Retrieved 2019-02-01 from http://www.cboe.com/micro/vix/vixwhite.pdf

Cheah, E. T., \& Fry, J. (2015). Speculative bubbles in Bitcoin markets? An empirical investigation into the fundamental value of Bitcoin. Economics Letters, 130, 32-36.

Chilson N. (2018). It's time for a FTC Blockchain Working Group. Federal Trade Commission [FTC]. Retrieved 2019-02-01 from FTC webpage: https://www.ftc.gov/newsevents/blogs/techftc/2018/03/its-time-ftc-blockchain-working-group

Ciaian, P., Rajcaniova, M., \& Kancs, D. A. (2016). The economics of Bitcoin price formation. Applied Economics, 48(19), 1799-1815.

CoinMetrics (2018). On data and certainty. [Blog post]. Retrieved 2019-01-02 from https://CoinMetrics.io/on-data-and-certainty/

Corbet, S., Lucey, B., Urquhart, A., \& Yarovaya, L. (2018). Cryptocurrencies as a financial asset: A systematic analysis. International Review of Financial Analysis.

Dabrowski, M. \& Janikowski, L. (2018) Virtual currencies and central banks monetary policy: challenges ahead (Monetary Dialogue July 2018). Retrieved 2019-02-27 from 
Dwyer, G. P. (2015). The economics of Bitcoin and similar private digital currencies. Journal of Financial Stability, 17, 81-91.

Dyhrberg, A. H. (2016). Bitcoin, gold and the dollar-A GARCH volatility analysis. Finance Research Letters, 16, 85-92.

Economic Policy Uncertainty (2019). Methodology. Retrieved 2019-03-01 from https://www.policyuncertainty.com/methodology.html

European Central Bank [ECB]. (2012). Virtual currency schemes. Frankfurt am Main. Retrieved 2019-01-02 from https://www.ecb.europa.eu/pub/pdf/other/virtualcurrencyschemes201210en.pdf

European Central Bank [ECB]. (2015). Virtual currency schemes - a further analysis. Frankfurt am Main. Retrieved 2019-01-02 from https://www.ecb.europa.eu/pub/pdf/other/virtualcurrencyschemesen.pdf

Foley, S., Karlsen, J. R., \& Putniņš, T. J. (2018). Sex, drugs, and Bitcoin: how much illegal activity is financed through cryptocurrencies?. Review of Financial Studies, Forthcoming.

Gans, J. S., \& Halaburda, H. (2015). Some economics of private digital currency. In Economic Analysis of the Digital Economy (pp. 257-276). University of Chicago Press.

Gandal, N., \& Halaburda, H. (2014). Competition in the cryptocurrency market. [Working paper]. Retrieved 2018-01-01 from https://www.bankofcanada.ca/wp-content/uploads/2014/08/wp2014-33.pdf

Gauraha, N. (2018). Introduction to the LASSO. Resonance, 23(4), 439-464.

GoogleTrends (2019) Interest over time, retrieved 2019-05-08 from https://trends.Google.com/trends/explore

Hastie, T., Tibshirani, R., \& Friedman, J. (2001). The elements of statistical learning : Data mining, inference, and prediction (Springer series in statistics). New York: Springer.

Hyndman, R.J., \& Athanasopoulos, G. (2018) Forecasting: principles and practice, 2nd edition, OTexts: Melbourne, Australia. OTexts.com/fpp2. Accessed on 2019-06-07.

Kristoufek, L. (2013). Bitcoin meets Google Trends and Wikipedia: Quantifying the relationship between phenomena of the Internet era. Scientific reports, 3, 3415.

Li, X., \& Wang, C. A. (2017). The technology and economic determinants of cryptocurrency exchange rates: The case of Bitcoin. Decision Support Systems, 95, 49-60.

Minto, A., Voelkerling, M., \& Wulff, M. (2017). Separating apples from oranges: identifying threats to financial stability originating from FinTech. Capital Markets Law Journal, 12(4), 428-465.

OECD. (2018). Blockchain Technology and Competition Policy. Issues paper provided by the OECD. Retrieved 2019-02-01 from:

http://www.oecd.org/competition/blockchain-and-competition-policy.htm

Panagiotidis, T., Stengos, T., \& Vravosinos, O. (2018). On the determinants of Bitcoin returns: A LASSO approach. Finance Research Letters, 27, 235-240. 
Pieters, G. C. (2017). The Potential Impact of Decentralized Virtual Currency on Monetary Policy. Federal Reserve Bank of Dallas - Globalization and Monetary Policy Institute 2016 Annual Report. Available at SSRN: https://ssrn.com/abstract=2976515

Polasik, M., Piotrowska, A. I., Wisniewski, T. P., Kotkowski, R., \& Lightfoot, G. (2015). Price fluctuations and the use of Bitcoin: An empirical inquiry. International Journal of Electronic Commerce, 20(1), 9-49.

Quandl (2019a) Gold Prices (Daily) - Currency USD. Retrieved 2019-05-13 from https://www.quandl.com/data/WGC/GOLD_DAILY_USD

Quandl (2019b) OPEC Crude Oil Price. Retrieved 2019-05-13 from https://www.quandl.com/data/OPEC/ORB

Tibshirani, R. (1996). Regression shrinkage and selection via the lasso. Journal of the Royal Statistical Society: Series B (Methodological), 58(1), 267-288.

Tsay, R. (2014). Analysis of financial time series. (3.rd ed.). Wiley.

Verbeek, M. (2017). A guide to modern econometrics. (5.th ed.). Wiley.

Waldman, D., \& Jensen, E. (2013). Industrial organization : Theory and practice (Pearson new international ed., The Pearson series in economics). Boston: Pearson.

Østbye, P. (2018). Will Regulation Change Cryptocurrency Protocols? Available at SSRN 3159479. 
Appendix A: How are tokens created? Results divided by time period

Table A1: Determinants of returns for each cryptocurrency in the crash time period, ranging from 2013-10-02 to 2017-01-03

\begin{tabular}{|c|c|c|c|c|c|c|c|c|c|}
\hline & & NEO & XRP & BTC & LTC & ETC & $\mathrm{XMR}$ & ETH & XLM \\
\hline & Token creation & \multicolumn{2}{|c|}{ Fixed supply } & \multicolumn{4}{|c|}{ Rise up to cap } & \multicolumn{2}{|c|}{ Rise indefinately } \\
\hline H1 & circulating & $\mathrm{n} / \mathrm{a}$ & $\mathrm{n} / \mathrm{a}$ & 325.4565 & -228.5603 & -23.7102 & & & $\mathrm{n} / \mathrm{a}$ \\
\hline $\mathrm{H} 2$ & averagedifficulty & $\mathrm{n} / \mathrm{a}$ & $\mathrm{n} / \mathrm{a}$ & & 0.0255 & 0.3563 & 0.0245 & 0.0504 & $\mathrm{n} / \mathrm{a}$ \\
\hline H3 & exchangevolumeusd & & & -0.0079 & 0.0029 & & & & \\
\hline \multirow[b]{12}{*}{ H5 } & L.LreturnADA & & & & & & & & \\
\hline & L.LreturnBCH & & & & 0.1670 & & & & \\
\hline & L.LreturnBTC & & & $\mathrm{n} / \mathrm{a}$ & -0.0970 & & 0.3111 & & \\
\hline & L.LreturnEOS & & & & & $\mathrm{n} / \mathrm{a}$ & & & \\
\hline & L.LreturnETC & & 0.0406 & -0.0489 & & & -0.1293 & & \\
\hline & L.LreturnETH & & & -0.0345 & -0.0513 & 0.1925 & -0.0299 & $n / a$ & \\
\hline & L.LreturnLTC & & & -0.1149 & $n / a$ & 0.1207 & -0.0085 & & \\
\hline & L.LreturnNEO & $n / a$ & -0.0045 & 0.0133 & 0.0149 & 0.0006 & 0.0476 & & \\
\hline & \begin{tabular}{|l|} 
L.LreturnTRXX \\
\end{tabular} & & & & & & & & \\
\hline & L.LreturnXLM & & & -0.0008 & 0.0359 & & 0.0642 & & $\mathrm{n} / \mathrm{a}$ \\
\hline & L.LreturnXMR & & & 0.0276 & 0.1037 & & $\mathrm{n} / \mathrm{a}$ & & \\
\hline & L.LreturnXRP & & $\mathrm{n} / \mathrm{a}$ & -0.0281 & -0.0698 & & -0.2466 & & \\
\hline $\mathrm{H6}$ & google & -1.2240 & & -0.0220 & -0.0285 & & 0.2425 & & \\
\hline \multirow[b]{7}{*}{ H7 } & SP500 & & & & 2.9806 & & & & \\
\hline & NYSE & & -1.3087 & -0.3232 & -2.1330 & & 3.1962 & & \\
\hline & AMEX & & -0.0506 & 0.5891 & 0.3526 & & -2.1006 & & \\
\hline & NASDAQ & & & & & -1.0990 & 1.6826 & & \\
\hline & NIKKEI & & & -0.2899 & & & -0.5428 & & \\
\hline & SSE & & 0.0314 & 0.0178 & 0.5616 & -0.7596 & -1.1494 & & \\
\hline & Oil & & & 0.1275 & 0.1667 & -0.0753 & 0.8530 & & \\
\hline \multirow[b]{6}{*}{ H8 } & USEPU & & & 0.4539 & 0.4923 & & -0.2512 & & \\
\hline & EEPU & & & & & & -0.4551 & & \\
\hline & CEPU & & -0.0509 & -0.1296 & -0.2573 & & -0.3669 & & \\
\hline & VIX & & & 0.1028 & 0.1440 & 0.0005 & -0.3088 & & \\
\hline & $\mathrm{VXO}$ & & & 0.0168 & -0.0426 & & & 0.0138 & \\
\hline & VXN & & -0.1007 & -0.1055 & -0.0253 & & 0.8421 & & \\
\hline \multirow[b]{5}{*}{ H9 } & exchus & & & -1.4200 & -3.2752 & & 16.8547 & & \\
\hline & exjpus & & & & -0.2349 & & -1.9856 & & \\
\hline & exukus & & & -0.3908 & -0.35833 & -0.3109 & 1.8826 & & \\
\hline & exeuus & & & 0.8559 & 1.0145 & -0.5602 & 0.0268 & & \\
\hline & Gold & & & 0.0411 & -0.1064 & & 0.1453 & & \\
\hline \multirow[t]{2}{*}{ Time trend } & Trend & & & 0.0002 & 0.0003 & & 0.0005 & & \\
\hline & Constant & -0.0033 & 0.0018 & -0.3044 & -0.1956 & 0.0576 & -0.5105 & -0.0029 & 0.0019 \\
\hline
\end{tabular}


Table A2: Determinants of returns for each cryptocurrency in the Recent time period, ranging from 2017-01-04 to 2017-06-23

\begin{tabular}{|c|c|c|c|c|c|c|c|c|c|}
\hline & & NEO & XRP & BTC & LTC & ETC & XMR & ETH & $\mathrm{XLM}$ \\
\hline & Token creation & \multicolumn{2}{|c|}{ Fixed supply } & \multicolumn{4}{|c|}{ Rise up to cap } & \multicolumn{2}{|c|}{ Rise indefinately } \\
\hline H1 & circulating & $\mathrm{n} / \mathrm{a}$ & $\mathrm{n} / \mathrm{a}$ & 264.5955 & & & -20.8651 & & $\mathrm{n} / \mathrm{a}$ \\
\hline $\mathrm{H} 2$ & averagedifficulty & $\mathrm{n} / \mathrm{a}$ & $\mathrm{n} / \mathrm{a}$ & 0.1746 & & 0.4811 & & 0.5232 & $\mathrm{n} / \mathrm{a}$ \\
\hline $\mathrm{H3}$ & exchangevolumeusd & 0.0114 & & 0.0272 & & & 0.0173 & & 0.0362 \\
\hline \multirow[b]{12}{*}{ H5 } & L.LreturnADA & & & & & & & & \\
\hline & L.LreturnBCH & -0.6685 & & & & & & & \\
\hline & L.LreturnBTC & & -0.0551 & $\mathrm{n} / \mathrm{a}$ & & & 0.0125 & & -0.0844 \\
\hline & L.LreturnEOS & & & & & & & & \\
\hline & L.LreturnETC & -0.1579 & & -0.0492 & & $n / a$ & & & -0.3593 \\
\hline & \begin{tabular}{|l|} 
L.LreturnETH \\
\end{tabular} & -0.1246 & & 0.0237 & & & 0.0854 & $\mathrm{n} / \mathrm{a}$ & 0.1929 \\
\hline & L.LreturnLTC & 0.0820 & 0.1250 & 0.0568 & $\mathrm{n} / \mathrm{a}$ & & & & 0.1288 \\
\hline & L.LreturnNEO & $\mathrm{n} / \mathrm{a}$ & & 0.0178 & & & & & 0.0751 \\
\hline & L.LreturnTRX & & & & & & & & \\
\hline & \begin{tabular}{|l} 
L.LreturnXLM \\
\end{tabular} & -0.2208 & 0.0109 & -0.0062 & & & -0.0322 & -0.0586 & $\mathrm{n} / \mathrm{a}$ \\
\hline & L.LreturnXMR & 0.9912 & & -0.0629 & & 0.1755 & $\mathrm{n} / \mathrm{a}$ & 0.2136 & 0.1215 \\
\hline & L.LreturnXRP & 0.1287 & $\mathrm{n} / \mathrm{a}$ & -0.0078 & & & & & -0.0204 \\
\hline H6 & google & 2.5363 & 0.6083 & 0.2884 & & & 0.2115 & & 0.2249 \\
\hline \multirow[b]{7}{*}{ H7 } & SP500 & 33.7203 & & 8.8993 & & & & & \\
\hline & NYSE & -19.0948 & & -4.2253 & & & & & \\
\hline & AMEX & -7.8270 & -1.2387 & -5.5482 & & & & & -1.7066 \\
\hline & NASDAQ & -4.0599 & & 1.209 & & & & & \\
\hline & NIKKEI & 6.6608 & 0.8585 & 1.0886 & & & 0.6866 & & 0.8988 \\
\hline & SSE & -3.2350 & & 0.7494 & & & & -0.6770 & \\
\hline & Oil & 0.3459 & & -0.0479 & & & 0.5207 & & \\
\hline \multirow[b]{6}{*}{ H8 } & USEPU & 2.1338 & & 0.4413 & & & & & -0.7911 \\
\hline & EEPU & -1.8207 & & 0.0478 & & & & 0.3092 & 1.6427 \\
\hline & CEPU & 0.1624 & & 0.0365 & & & & & \\
\hline & $\mathrm{VIX}$ & 0.2614 & & -0.1642 & & & & & \\
\hline & VXO & -0.5508 & 0.2003 & -0.1452 & & & -0.0371 & -0.1088 & \\
\hline & VXN & 0.4876 & 0.1176 & 0.3613 & & & & -0.0096 & 0.1283 \\
\hline \multirow[b]{5}{*}{ H9 } & exchus & 0.7304 & 0.2850 & -5.0706 & & & & -3.5882 & 6.5403 \\
\hline & exjpus & -7.1241 & 1.0674 & -1.1492 & & & & & -0.3492 \\
\hline & exukus & 8.2117 & & 1.0112 & & & & & \\
\hline & exeuus & -1.8914 & & 3.1579 & & & & & \\
\hline & Gold & 0.4650 & & 0.1946 & & & & & 0.2939 \\
\hline \multirow[t]{2}{*}{ Time trend } & Trend & 0.0005 & & & & & & & 0.0001 \\
\hline & Constant & -0.5674 & 0.0193 & -0.0280 & 0.0137 & 0.0102 & 0.0133 & 0.0137 & -0.1683 \\
\hline
\end{tabular}


Table A3: Determinants of returns for each cryptocurrency in the Current time period, ranging from 2017-01-04 to 2019-01-01

\begin{tabular}{|c|c|c|c|c|c|c|c|c|c|c|c|c|c|}
\hline & & NEO & XRP & ADA & TRX & BTC & LTC & ETC & XMR & $\mathrm{BCH}$ & ETH & $\mathrm{XLM}$ & EOS \\
\hline & Token creation & \multicolumn{4}{|c|}{$\begin{array}{l}\text { Fixed supply } \\
\end{array}$} & \multicolumn{5}{|c|}{ Rise up to cap } & \multicolumn{3}{|c|}{ Rise indefinately } \\
\hline H1 & circulating & $n / a$ & $n / a$ & $\mathrm{n} / \mathrm{a}$ & $n / a$ & & & & & & & $n / a$ & $n / a$ \\
\hline $\mathrm{H} 2$ & averagedifficulty & $n / a$ & $n / a$ & $\mathrm{n} / \mathrm{a}$ & $n / a$ & & & 0.2264 & & 0.0730 & 0.0054 & $\mathrm{n} / \mathrm{a}$ & $n / a$ \\
\hline $\mathrm{H3}$ & exchangevolumeusd & 0.0090 & & & & & & & & & & & \\
\hline \multirow[b]{12}{*}{ H5 } & L..LreturnADA & & -0.0455 & $\mathrm{n} / \mathrm{a}$ & & & & & & & & & \\
\hline & \begin{tabular}{|l|} 
L.LreturnBCH \\
\end{tabular} & -0.0013 & & & & & & & & $\mathrm{n} / \mathrm{a}$ & & & \\
\hline & L.LreturnBTC & & -0.0168 & & & $n / a$ & & & & & & & \\
\hline & L.LreturnEOS & & & & & & & & & & & & $n / a$ \\
\hline & L.LreturnETC & & -0.0450 & & -0.0667 & & & $\mathrm{n} / \mathrm{a}$ & & & & & \\
\hline & L.LreturnETH & & & & & & & & & & $\mathrm{n} / \mathrm{a}$ & & \\
\hline & L.LreturnLTC & & & & & & $n / a$ & & & & & & 0.0530 \\
\hline & \begin{tabular}{|l|l|} 
L.LreturnNEO \\
\end{tabular} & $\mathrm{n} / \mathrm{a}$ & & & & & & & & & & & \\
\hline & \begin{tabular}{|l} 
L.LreturnTRX \\
\end{tabular} & 0.0279 & 0.1056 & & $\mathrm{n} / \mathrm{a}$ & & & & & & & & \\
\hline & L.LreturnXLM & 0.0065 & & & 0.0778 & & & & & & & $n / a$ & \\
\hline & L.LreturnXMR & -0.0492 & & & & & & & $n / a$ & & & & \\
\hline & L.LreturnXRP & -0.0563 & $\mathrm{n} / \mathrm{a}$ & & & & & & & & -0.0086 & & -0.0399 \\
\hline H6 & google & 0.5983 & 0.0290 & & & & & & & & 0.0824 & & 0.6986 \\
\hline \multirow[b]{7}{*}{ H7 } & SP500 & & & & & & & & & & & & \\
\hline & NYSE & & & & & & & & & & & & \\
\hline & AMEX & 0.3361 & & & & & & & & & & & 0.0949 \\
\hline & NASDAQ & & & & & & & & & & & & \\
\hline & NIKKEI & & & & & & & & & & & & \\
\hline & SSE & & & & & & & & & & & & -0.1345 \\
\hline & Oil & & & & & & & & & & & & \\
\hline \multirow[b]{6}{*}{ H8 } & USEPU & & & & & & & & & & & & \\
\hline & EEPU & & 0.4699 & & & & & & & & & & \\
\hline & CEPU & -0.0743 & & & & & & & & & & & -0.1462 \\
\hline & VIX & & -0.0381 & & & & & & & & & & \\
\hline & $\mathrm{VXO}$ & & & & & & & & & & & & \\
\hline & VXN & 0.0436 & & & & & & & & & & & \\
\hline \multirow[b]{5}{*}{ H9 } & exchus & & & & & & & & & & & & \\
\hline & exjpus & & & & & & & & & & & & \\
\hline & exukus & & & & & & & & & & & & \\
\hline & exeuus & -0.5074 & & & & & & & & & & & -0.5168 \\
\hline & Gold & & & & & & & & & & & & \\
\hline \multirow[t]{2}{*}{ Time trend } & Trend & & & & & & & & & & & & \\
\hline & Constant & -0.0021 & 0.0004 & 0.0021 & 0.0042 & 0.0006 & 0.0002 & -0.0018 & -0.0009 & -0.0018 & -0.0013 & - & 0.0035 \\
\hline
\end{tabular}


Appendix B: How are tokens distributed and transactions validated? Results divided by time period

Table B1: Determinants of returns for each cryptocurrency in the Crash time period, ranging from 2013-10-02 to 2017-01-03

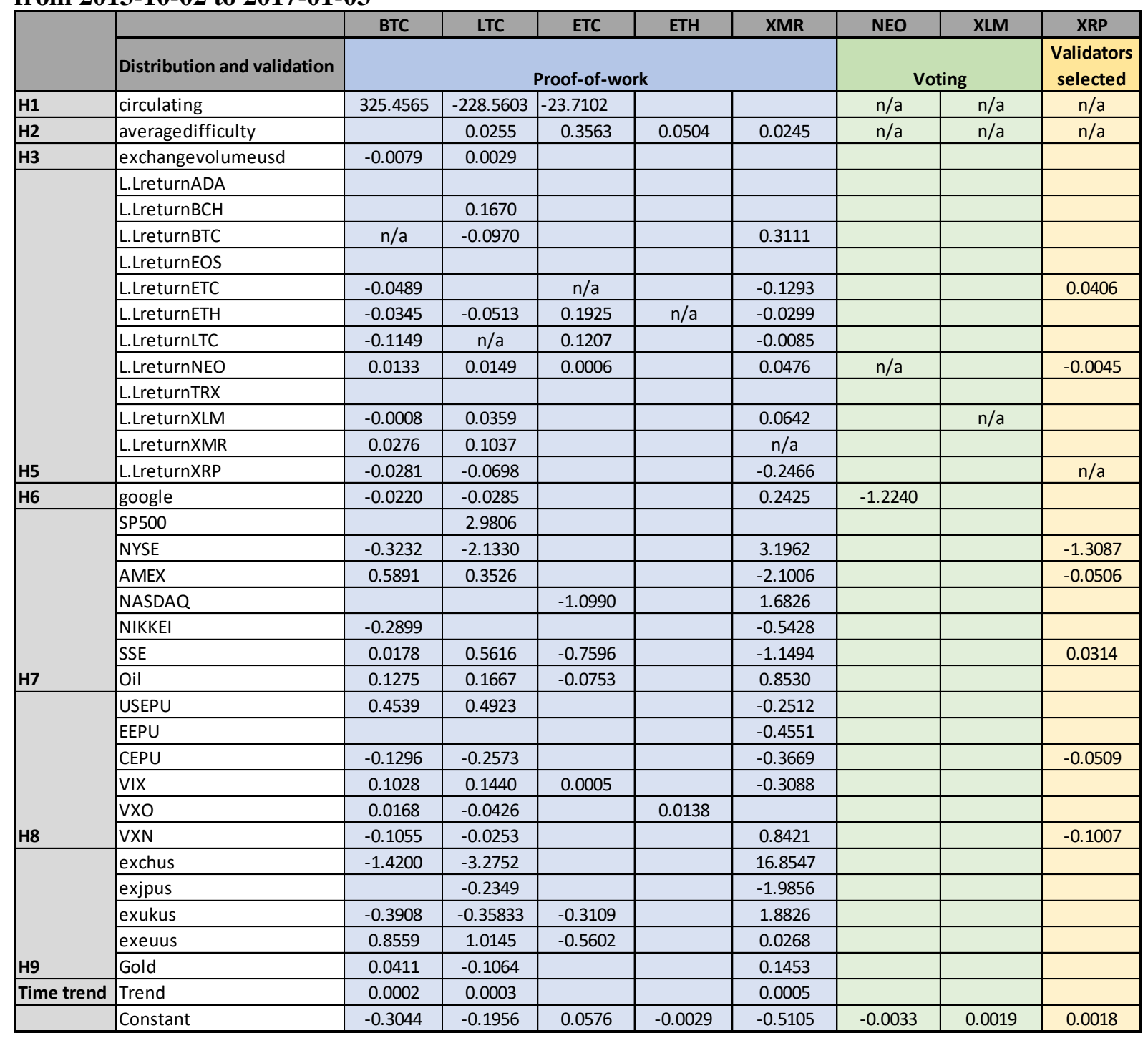


Table B2: Determinants of returns for each cryptocurrency in the Recent time period, ranging from 2017-01-04 to 2017-06-23

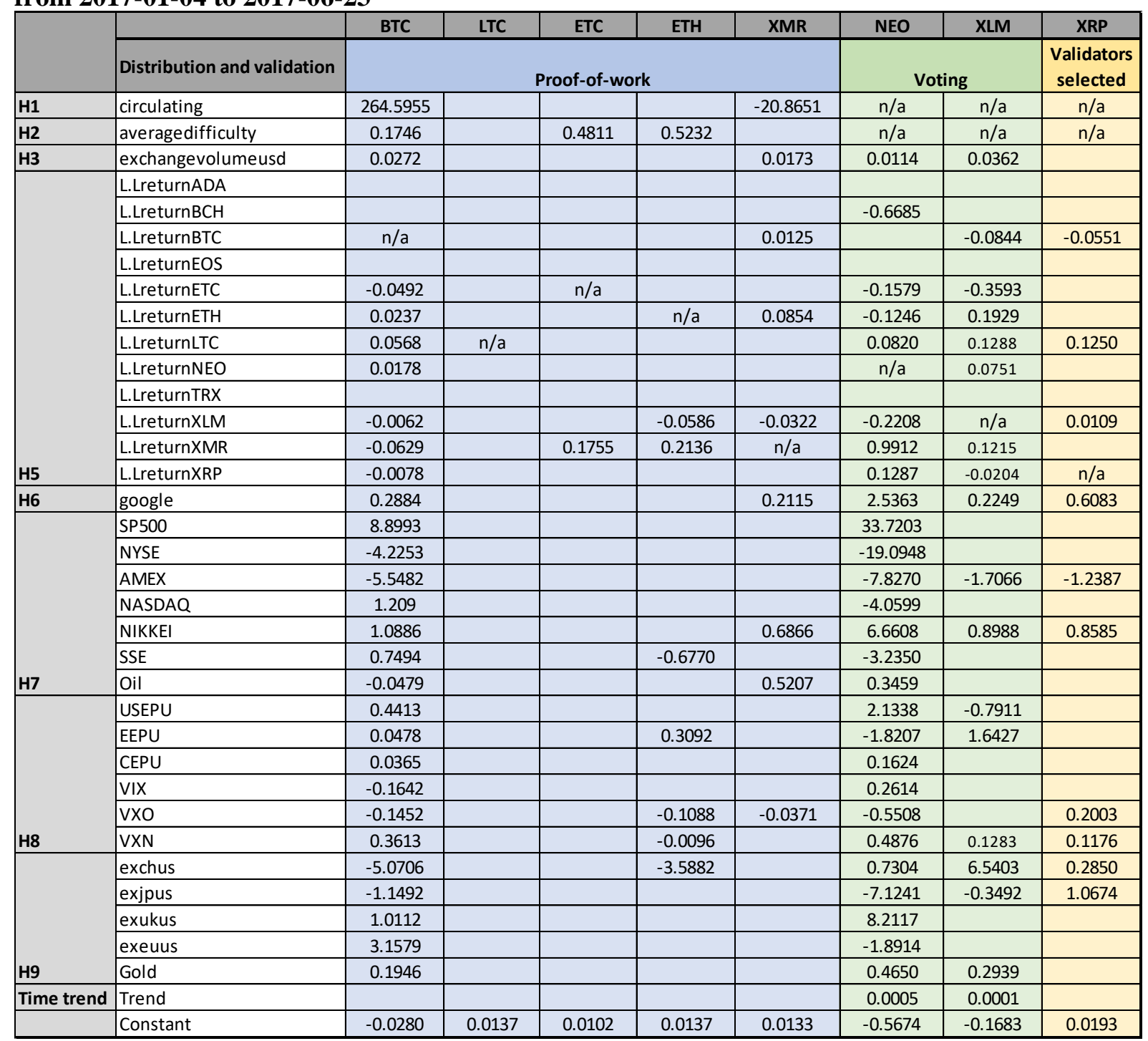


Table B3: Determinants of returns for each cryptocurrency in the Current time period, ranging from 2017-01-04 to 2019-01-01

\begin{tabular}{|c|c|c|c|c|c|c|c|c|c|c|c|c|c|}
\hline & & BTC & LTC & ETC & ETH & XMR & BCH & TRX & NEO & EOS & $\mathrm{XLM}$ & \multirow{3}{*}{\begin{tabular}{|c|} 
XRP \\
$\begin{array}{c}\text { Validators } \\
\text { selected }\end{array}$ \\
$\mathrm{n} / \mathrm{a}$ \\
\end{tabular}} & \multirow{3}{*}{\begin{tabular}{|c|} 
ADA \\
$\begin{array}{c}\text { Proof-of- } \\
\text { stake }\end{array}$ \\
n/a \\
\end{tabular}} \\
\hline & Distribution and validation & \multicolumn{7}{|c|}{ Proof-of-work } & \multicolumn{3}{|c|}{ Voting } & & \\
\hline H1 & circulating & & & & & & & $n / a$ & $n / a$ & $n / a$ & $n / a$ & & \\
\hline H2 & averagedifficulty & & & 0.2264 & 0.0054 & & 0.0730 & $n / a$ & $\mathrm{n} / \mathrm{a}$ & $n / a$ & $n / a$ & $n / a$ & $n / a$ \\
\hline H3 & exchangevolumeusd & & & & & & & & 0.0090 & & & & \\
\hline & L.LreturnADA & & & & & & & & & & & -0.0455 & $n / a$ \\
\hline & L.LreturnBCH & & & & & & $n / a$ & & -0.0013 & & & & \\
\hline & L.LreturnBTC & $n / a$ & & & & & & & & & & -0.0168 & \\
\hline & L.LreturnEOS & & & & & & & & & $n / a$ & & & \\
\hline & L.LreturnETC & & & $n / a$ & & & & -0.0667 & & & & -0.0450 & \\
\hline & L.LreturnETH & & & & $n / a$ & & & & & & & & \\
\hline & L.LreturnLTC & & $n / a$ & & & & & & & 0.0530 & & & \\
\hline & L.LreturnNEO & & & & & & & & $n / a$ & & & & \\
\hline & L.LreturnTRX & & & & & & & $n / a$ & 0.0279 & & & 0.1056 & \\
\hline & L.LreturnXLM & & & & & & & 0.0778 & 0.0065 & & $n / a$ & & \\
\hline & L.LreturnXMR & & & & & $n / a$ & & & -0.0492 & & & & \\
\hline H5 & L.LreturnXRP & & & & -0.0086 & & & & -0.0563 & -0.0399 & & $n / a$ & \\
\hline \multirow[t]{7}{*}{ H6 } & google & & & & 0.0824 & & & & 0.5983 & 0.6986 & & 0.0290 & \\
\hline & SP500 & & & & & & & & & & & & \\
\hline & NYSE & & & & & & & & & & & & \\
\hline & AMEX & & & & & & & & 0.3361 & 0.0949 & & & \\
\hline & NASDAQ & & & & & & & & & & & & \\
\hline & NIKKEI & & & & & & & & & & & & \\
\hline & SSE & & & & & & & & & -0.1345 & & & \\
\hline \multirow[t]{6}{*}{$\mathrm{H} 7$} & Oil & & & & & & & & & & & & \\
\hline & USEPU & & & & & & & & & & & & \\
\hline & EEPU & & & & & & & & & & & 0.4699 & \\
\hline & CEPU & & & & & & & & -0.0743 & -0.1462 & & & \\
\hline & $\mathrm{VIX}$ & & & & & & & & & & & -0.0381 & \\
\hline & $\mathrm{VXO}$ & & & & & & & & & & & & \\
\hline \multirow[t]{5}{*}{ H8 } & VXN & & & & & & & & 0.0436 & & & & \\
\hline & exchus & & & & & & & & & & & & \\
\hline & exjpus & & & & & & & & & & & & \\
\hline & exukus & & & & & & & & & & & & \\
\hline & exeuus & & & & & & & & -0.5074 & -0.5168 & & & \\
\hline H9 & Gold & & & & & & & & & & & & \\
\hline \multirow{2}{*}{ Time trend } & Trend & & & & & & & & & & & & \\
\hline & Constant & 0.0006 & 0.0002 & -0.0018 & -0.0013 & -0.0009 & -0.0018 & 0.0042 & -0.0021 & 0.0035 & & 0.0004 & 0.0021 \\
\hline
\end{tabular}


Appendix C: What is the target market for the token? Results divided by time period

Table C1: Determinants of returns for each cryptocurrency in the Crash time period, ranging from 2013-10-02 to 2017-01-03

\begin{tabular}{|c|c|c|c|c|c|c|c|c|c|}
\hline & & BTC & LTC & ETC & ETH & XMR & NEO & XLM & XRP \\
\hline & Target market & \multicolumn{6}{|c|}{ Generic } & \multicolumn{2}{|c|}{ Business-oriented } \\
\hline H1 & circulating & 325.4565 & -228.5603 & -23.7102 & & & $\mathrm{n} / \mathrm{a}$ & $\mathrm{n} / \mathrm{a}$ & $\mathrm{n} / \mathrm{a}$ \\
\hline $\mathrm{H} 2$ & averagedifficulty & & 0.0255 & 0.3563 & 0.0504 & 0.0245 & $\mathrm{n} / \mathrm{a}$ & $\mathrm{n} / \mathrm{a}$ & $\mathrm{n} / \mathrm{a}$ \\
\hline H3 & exchangevolumeusd & -0.0079 & 0.0029 & & & & & & \\
\hline \multirow[b]{12}{*}{ H5 } & L.LreturnADA & & & & & & & & \\
\hline & L.LreturnBCH & & 0.1670 & & & & & & \\
\hline & L.LreturnBTC & $\mathrm{n} / \mathrm{a}$ & -0.0970 & & & 0.3111 & & & \\
\hline & L.LreturnEOS & & & & & & & & \\
\hline & L.LreturnETC & -0.0489 & & $\mathrm{n} / \mathrm{a}$ & & -0.1293 & & & 0.0406 \\
\hline & L.LreturnETH & -0.0345 & -0.0513 & 0.1925 & $\mathrm{n} / \mathrm{a}$ & -0.0299 & & & \\
\hline & L.LreturnLTC & -0.1149 & $\mathrm{n} / \mathrm{a}$ & 0.1207 & & -0.0085 & & & \\
\hline & L.LreturnNEO & 0.0133 & 0.0149 & 0.0006 & & 0.0476 & $\mathrm{n} / \mathrm{a}$ & & -0.0045 \\
\hline & L.LreturnTRX & & & & & & & & \\
\hline & L.LreturnXLM & -0.0008 & 0.0359 & & & 0.0642 & & $\mathrm{n} / \mathrm{a}$ & \\
\hline & L.LreturnXMR & 0.0276 & 0.1037 & & & $\mathrm{n} / \mathrm{a}$ & & & \\
\hline & L.LreturnXRP & -0.0281 & -0.0698 & & & -0.2466 & & & $\mathrm{n} / \mathrm{a}$ \\
\hline H6 & google & -0.0220 & -0.0285 & & & 0.2425 & -1.2240 & & \\
\hline \multirow[b]{7}{*}{ H7 } & SP500 & & 2.9806 & & & & & & \\
\hline & NYSE & -0.3232 & -2.1330 & & & 3.1962 & & & -1.3087 \\
\hline & AMEX & 0.5891 & 0.3526 & & & -2.1006 & & & -0.0506 \\
\hline & NASDAQ & & & -1.0990 & & 1.6826 & & & \\
\hline & NIKKEI & -0.2899 & & & & -0.5428 & & & \\
\hline & SSE & 0.0178 & 0.5616 & -0.7596 & & -1.1494 & & & 0.0314 \\
\hline & Oil & 0.1275 & 0.1667 & -0.0753 & & 0.8530 & & & \\
\hline \multirow[b]{6}{*}{ H8 } & USEPU & 0.4539 & 0.4923 & & & -0.2512 & & & \\
\hline & EEPU & & & & & -0.4551 & & & \\
\hline & CEPU & -0.1296 & -0.2573 & & & -0.3669 & & & -0.0509 \\
\hline & $\mathrm{VIX}$ & 0.1028 & 0.1440 & 0.0005 & & -0.3088 & & & \\
\hline & $\mathrm{VXO}$ & 0.0168 & -0.0426 & & 0.0138 & & & & \\
\hline & VXN & -0.1055 & -0.0253 & & & 0.8421 & & & -0.1007 \\
\hline \multirow[b]{5}{*}{ H9 } & exchus & -1.4200 & -3.2752 & & & 16.8547 & & & \\
\hline & exjpus & & -0.2349 & & & -1.9856 & & & \\
\hline & exukus & -0.3908 & -0.35833 & -0.3109 & & 1.8826 & & & \\
\hline & exeuus & 0.8559 & 1.0145 & -0.5602 & & 0.0268 & & & \\
\hline & Gold & 0.0411 & -0.1064 & & & 0.1453 & & & \\
\hline \multirow[t]{2}{*}{ Time trend } & Trend & 0.0002 & 0.0003 & & & 0.0005 & & & \\
\hline & Constant & -0.3044 & -0.1956 & 0.0576 & -0.0029 & -0.5105 & -0.0033 & 0.0019 & 0.0018 \\
\hline
\end{tabular}


Table C2: Determinants of returns for each cryptocurrency in the Recent time period, ranging from 2017-01-04 to 2017-06-23

\begin{tabular}{|c|c|c|c|c|c|c|c|c|c|}
\hline & & BTC & LTC & ETC & ETH & XMR & NEO & XLM & XRP \\
\hline & Target market & \multicolumn{6}{|c|}{ Generic } & \multicolumn{2}{|c|}{ Business-oriented } \\
\hline H1 & circulating & 264.5955 & & & & -20.8651 & $\mathrm{n} / \mathrm{a}$ & $\mathrm{n} / \mathrm{a}$ & $\mathrm{n} / \mathrm{a}$ \\
\hline $\mathrm{H} 2$ & averagedifficulty & 0.1746 & & 0.4811 & 0.5232 & & $\mathrm{n} / \mathrm{a}$ & $\mathrm{n} / \mathrm{a}$ & $\mathrm{n} / \mathrm{a}$ \\
\hline H3 & exchangevolumeusd & 0.0272 & & & & 0.0173 & 0.0114 & 0.0362 & \\
\hline \multirow[b]{12}{*}{ H5 } & L.LreturnADA & & & & & & & & \\
\hline & L.LreturnBCH & & & & & & -0.6685 & & \\
\hline & L.LreturnBTC & $\mathrm{n} / \mathrm{a}$ & & & & 0.0125 & & -0.0844 & -0.0551 \\
\hline & L.LreturnEOS & & & & & & & & \\
\hline & L.LreturnETC & -0.0492 & & $n / a$ & & & -0.1579 & -0.3593 & \\
\hline & L.LreturnETH & 0.0237 & & & $\mathrm{n} / \mathrm{a}$ & 0.0854 & -0.1246 & 0.1929 & \\
\hline & L.LreturnLTC & 0.0568 & $\mathrm{n} / \mathrm{a}$ & & & & 0.0820 & 0.1288 & 0.1250 \\
\hline & L.LreturnNEO & 0.0178 & & & & & $\mathrm{n} / \mathrm{a}$ & 0.0751 & \\
\hline & L.LreturnTRX & & & & & & & & \\
\hline & L.LreturnXLM & -0.0062 & & & -0.0586 & -0.0322 & -0.2208 & $\mathrm{n} / \mathrm{a}$ & 0.0109 \\
\hline & L.LreturnXMR & -0.0629 & & 0.1755 & 0.2136 & $\mathrm{n} / \mathrm{a}$ & 0.9912 & 0.1215 & \\
\hline & L.LreturnXRP & -0.0078 & & & & & 0.1287 & -0.0204 & $\mathrm{n} / \mathrm{a}$ \\
\hline H6 & google & 0.2884 & & & & 0.2115 & 2.5363 & 0.2249 & 0.6083 \\
\hline \multirow[b]{7}{*}{ H7 } & SP500 & 8.8993 & & & & & 33.7203 & & \\
\hline & NYSE & -4.2253 & & & & & -19.0948 & & \\
\hline & AMEX & -5.5482 & & & & & -7.8270 & -1.7066 & -1.2387 \\
\hline & NASDAQ & 1.209 & & & & & -4.0599 & & \\
\hline & NIKKEI & 1.0886 & & & & 0.6866 & 6.6608 & 0.8988 & 0.8585 \\
\hline & SSE & 0.7494 & & & -0.6770 & & -3.2350 & & \\
\hline & Oil & -0.0479 & & & & 0.5207 & 0.3459 & & \\
\hline \multirow[b]{6}{*}{ H8 } & USEPU & 0.4413 & & & & & 2.1338 & -0.7911 & \\
\hline & EEPU & 0.0478 & & & 0.3092 & & -1.8207 & 1.6427 & \\
\hline & CEPU & 0.0365 & & & & & 0.1624 & & \\
\hline & $\mathrm{VIX}$ & -0.1642 & & & & & 0.2614 & & \\
\hline & VXO & -0.1452 & & & -0.1088 & -0.0371 & -0.5508 & & 0.2003 \\
\hline & VXN & 0.3613 & & & -0.0096 & & 0.4876 & 0.1283 & 0.1176 \\
\hline \multirow[b]{5}{*}{ H9 } & exchus & -5.0706 & & & -3.5882 & & 0.7304 & 6.5403 & 0.2850 \\
\hline & exjpus & -1.1492 & & & & & -7.1241 & -0.3492 & 1.0674 \\
\hline & exukus & 1.0112 & & & & & 8.2117 & & \\
\hline & exeuus & 3.1579 & & & & & -1.8914 & & \\
\hline & Gold & 0.1946 & & & & & 0.4650 & 0.2939 & \\
\hline \multirow[t]{2}{*}{ Time trend } & Trend & & & & & & 0.0005 & 0.0001 & \\
\hline & Constant & -0.0280 & 0.0137 & 0.0102 & 0.0137 & 0.0133 & -0.5674 & -0.1683 & 0.0193 \\
\hline
\end{tabular}


Table C3: Determinants of returns for each cryptocurrency in the Current time period, ranging from 2017-01-04 to 2019-01-01

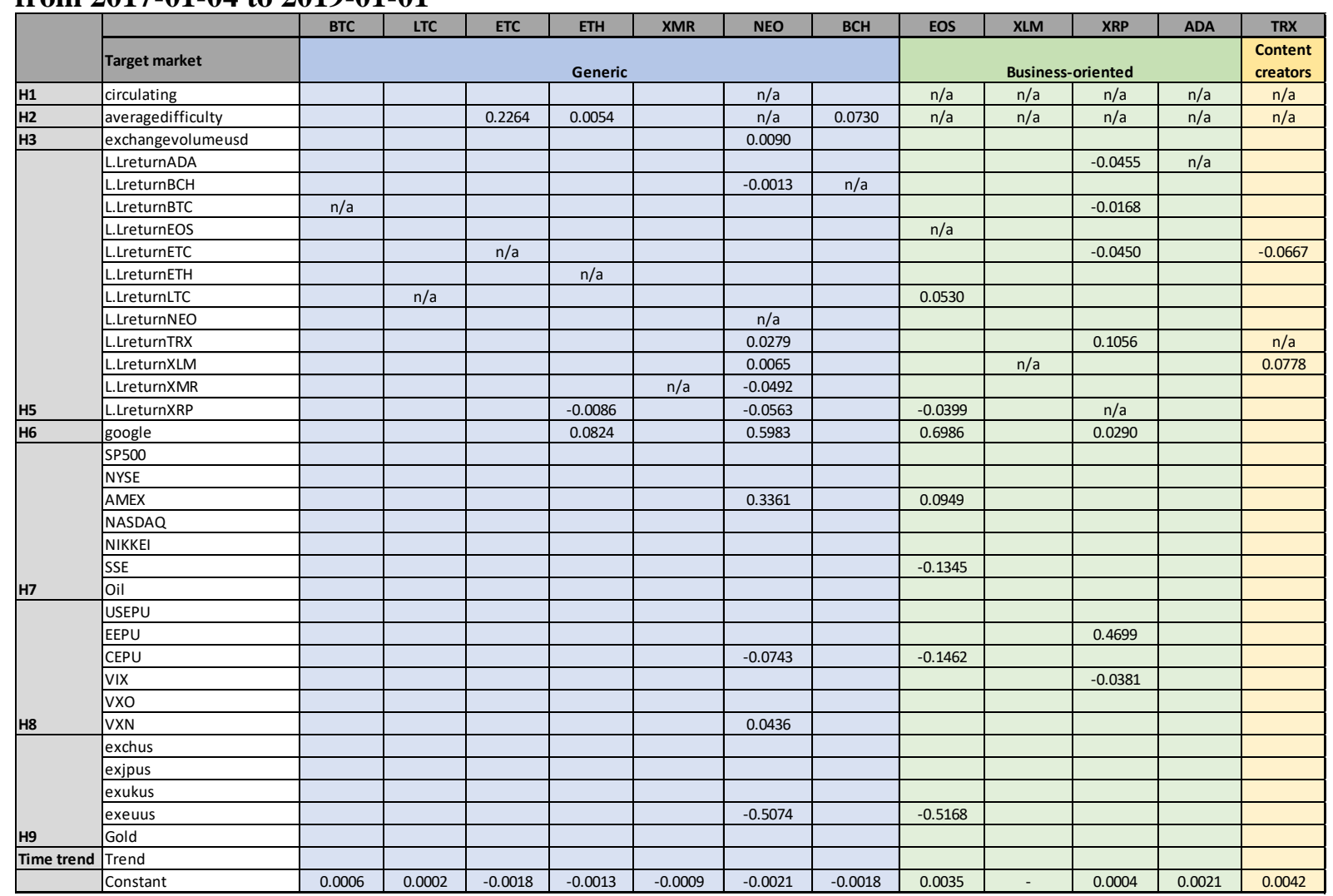


Appendix D: What is the token being used for? Results divided by time period Table D1: Determinants of returns for each cryptocurrency in the Crash time period, ranging from 2013-10-02 to 2017-01-03

\begin{tabular}{|c|c|c|c|c|c|c|c|c|c|}
\hline & & LTC & XRP & XMR & XLM & BTC & ETH & NEO & ETC \\
\hline & Token used for & \multicolumn{4}{|c|}{ Transaction } & \multicolumn{2}{|c|}{ Hybrid } & \multicolumn{2}{|c|}{ Applications } \\
\hline H1 & circulating & -228.5603 & $\mathrm{n} / \mathrm{a}$ & & $\mathrm{n} / \mathrm{a}$ & 325.4565 & & $\mathrm{n} / \mathrm{a}$ & -23.7102 \\
\hline $\mathrm{H} 2$ & averagedifficulty & 0.0255 & $\mathrm{n} / \mathrm{a}$ & 0.0245 & $\mathrm{n} / \mathrm{a}$ & & 0.0504 & $\mathrm{n} / \mathrm{a}$ & 0.3563 \\
\hline $\mathrm{H3}$ & exchangevolumeusd & 0.0029 & & & & -0.0079 & & & \\
\hline \multirow[b]{12}{*}{ H5 } & L.LreturnADA & & & & & & & & \\
\hline & L.LreturnBCH & 0.1670 & & & & & & & \\
\hline & L.LreturnBTC & -0.0970 & & 0.3111 & & $n / a$ & & & \\
\hline & L.LreturnEOS & & & & & & & & $\mathrm{n} / \mathrm{a}$ \\
\hline & L.LreturnETC & & 0.0406 & -0.1293 & & -0.0489 & & & \\
\hline & L.LreturnETH & -0.0513 & & -0.0299 & & -0.0345 & $\mathrm{n} / \mathrm{a}$ & & 0.1925 \\
\hline & L.LreturnLTC & $\mathrm{n} / \mathrm{a}$ & & -0.0085 & & -0.1149 & & & 0.1207 \\
\hline & L.LreturnNEO & 0.0149 & -0.0045 & 0.0476 & & 0.0133 & & $\mathrm{n} / \mathrm{a}$ & 0.0006 \\
\hline & L.LreturnTRX & & & & & & & & \\
\hline & L.LreturnXLM & 0.0359 & & 0.0642 & $\mathrm{n} / \mathrm{a}$ & -0.0008 & & & \\
\hline & L.LreturnXMR & 0.1037 & & $\mathrm{n} / \mathrm{a}$ & & 0.0276 & & & \\
\hline & L. LreturnXRP & -0.0698 & $\mathrm{n} / \mathrm{a}$ & -0.2466 & & -0.0281 & & & \\
\hline $\mathrm{H6}$ & google & -0.0285 & & 0.2425 & & -0.0220 & & -1.2240 & \\
\hline \multirow[b]{7}{*}{ H7 } & SP500 & 2.9806 & & & & & & & \\
\hline & NYSE & -2.1330 & -1.3087 & 3.1962 & & -0.3232 & & & \\
\hline & AMEX & 0.3526 & -0.0506 & -2.1006 & & 0.5891 & & & \\
\hline & NASDAQ & & & 1.6826 & & & & & -1.0990 \\
\hline & NIKKEI & & & -0.5428 & & -0.2899 & & & \\
\hline & SSE & 0.5616 & 0.0314 & -1.1494 & & 0.0178 & & & -0.7596 \\
\hline & Oil & 0.1667 & & 0.8530 & & 0.1275 & & & -0.0753 \\
\hline \multirow[b]{6}{*}{$\mathrm{H8}$} & USEPU & 0.4923 & & -0.2512 & & 0.4539 & & & \\
\hline & EEPU & & & -0.4551 & & & & & \\
\hline & CEPU & -0.2573 & -0.0509 & -0.3669 & & -0.1296 & & & \\
\hline & VIX & 0.1440 & & -0.3088 & & 0.1028 & & & 0.0005 \\
\hline & $\mathrm{VXO}$ & -0.0426 & & & & 0.0168 & 0.0138 & & \\
\hline & VXN & -0.0253 & -0.1007 & 0.8421 & & -0.1055 & & & \\
\hline \multirow[b]{5}{*}{ H9 } & exchus & -3.2752 & & 16.8547 & & -1.4200 & & & \\
\hline & exjpus & -0.2349 & & -1.9856 & & & & & \\
\hline & \begin{tabular}{|l|} 
exukus \\
\end{tabular} & -0.35833 & & 1.8826 & & -0.3908 & & & -0.3109 \\
\hline & exeuus & 1.0145 & & 0.0268 & & 0.8559 & & & -0.5602 \\
\hline & Gold & -0.1064 & & 0.1453 & & 0.0411 & & & \\
\hline \multirow[t]{2}{*}{ Time trend } & Trend & 0.0003 & & 0.0005 & & 0.0002 & & & \\
\hline & Constant & -0.1956 & 0.0018 & -0.5105 & 0.0019 & -0.3044 & -0.0029 & -0.0033 & 0.0576 \\
\hline
\end{tabular}


Table D2: Determinants of returns for each cryptocurrency in the Recent time period, ranging from 2017-01-04 to 2017-06-23

\begin{tabular}{|c|c|c|c|c|c|c|c|c|c|}
\hline & & LTC & XRP & XMR & $\mathrm{XLM}$ & BTC & ETH & NEO & ETC \\
\hline & Token used for & \multicolumn{4}{|c|}{ Transaction } & \multicolumn{2}{|c|}{ Hybrid } & \multicolumn{2}{|c|}{ Applications } \\
\hline H1 & circulating & & $\mathrm{n} / \mathrm{a}$ & -20.8651 & $\mathrm{n} / \mathrm{a}$ & 264.5955 & & $\mathrm{n} / \mathrm{a}$ & \\
\hline $\mathrm{H} 2$ & averagedifficulty & & $\mathrm{n} / \mathrm{a}$ & & $\mathrm{n} / \mathrm{a}$ & 0.1746 & 0.5232 & $\mathrm{n} / \mathrm{a}$ & 0.4811 \\
\hline H3 & exchangevolumeusd & & & 0.0173 & 0.0362 & 0.0272 & & 0.0114 & \\
\hline \multirow[b]{12}{*}{ H5 } & L.LreturnADA & & & & & & & & \\
\hline & \begin{tabular}{|l|} 
L.LreturnBCH \\
\end{tabular} & & & & & & & -0.6685 & \\
\hline & L.LreturnBTC & & -0.0551 & 0.0125 & -0.0844 & $n / a$ & & & \\
\hline & L.LreturnEOS & & & & & & & & \\
\hline & L.LreturnETC & & & & -0.3593 & -0.0492 & & -0.1579 & $\mathrm{n} / \mathrm{a}$ \\
\hline & L.LreturnETH & & & 0.0854 & 0.1929 & 0.0237 & $\mathrm{n} / \mathrm{a}$ & -0.1246 & \\
\hline & L.LreturnLTC & $n / a$ & 0.1250 & & 0.1288 & 0.0568 & & 0.0820 & \\
\hline & \begin{tabular}{|l|} 
L.LreturnNEO \\
\end{tabular} & & & & 0.0751 & 0.0178 & & $\mathrm{n} / \mathrm{a}$ & \\
\hline & L.LreturnTRX & & & & & & & & \\
\hline & L.LreturnXLM & & 0.0109 & -0.0322 & $\mathrm{n} / \mathrm{a}$ & -0.0062 & -0.0586 & -0.2208 & \\
\hline & L.LreturnXMR & & & $\mathrm{n} / \mathrm{a}$ & 0.1215 & -0.0629 & 0.2136 & 0.9912 & 0.1755 \\
\hline & \begin{tabular}{|l|} 
L.LreturnXRP \\
\end{tabular} & & $\mathrm{n} / \mathrm{a}$ & & -0.0204 & -0.0078 & & 0.1287 & \\
\hline H6 & google & & 0.6083 & 0.2115 & 0.2249 & 0.2884 & & 2.5363 & \\
\hline \multirow[b]{7}{*}{ H7 } & SP500 & & & & & 8.8993 & & 33.7203 & \\
\hline & NYSE & & & & & -4.2253 & & -19.0948 & \\
\hline & AMEX & & -1.2387 & & -1.7066 & -5.5482 & & -7.8270 & \\
\hline & NASDAQ & & & & & 1.209 & & -4.0599 & \\
\hline & NIKKEI & & 0.8585 & 0.6866 & 0.8988 & 1.0886 & & 6.6608 & \\
\hline & \begin{tabular}{|l|} 
SSE \\
\end{tabular} & & & & & 0.7494 & -0.6770 & -3.2350 & \\
\hline & Oil & & & 0.5207 & & -0.0479 & & 0.3459 & \\
\hline \multirow[b]{6}{*}{ H8 } & USEPU & & & & -0.7911 & 0.4413 & & 2.1338 & \\
\hline & EEPU & & & & 1.6427 & 0.0478 & 0.3092 & -1.8207 & \\
\hline & CEPU & & & & & 0.0365 & & 0.1624 & \\
\hline & VIX & & & & & -0.1642 & & 0.2614 & \\
\hline & $\mathrm{VXO}$ & & 0.2003 & -0.0371 & & -0.1452 & -0.1088 & -0.5508 & \\
\hline & VXN & & 0.1176 & & 0.1283 & 0.3613 & -0.0096 & 0.4876 & \\
\hline \multirow[b]{5}{*}{ H9 } & exchus & & 0.2850 & & 6.5403 & -5.0706 & -3.5882 & 0.7304 & \\
\hline & exjpus & & 1.0674 & & -0.3492 & -1.1492 & & -7.1241 & \\
\hline & exukus & & & & & 1.0112 & & 8.2117 & \\
\hline & exeuus & & & & & 3.1579 & & -1.8914 & \\
\hline & Gold & & & & 0.2939 & 0.1946 & & 0.4650 & \\
\hline \multirow[t]{2}{*}{ Time trend } & Trend & & & & 0.0001 & & & 0.0005 & \\
\hline & Constant & 0.0137 & 0.0193 & 0.0133 & -0.1683 & -0.0280 & 0.0137 & -0.5674 & 0.0102 \\
\hline
\end{tabular}


Table D3: Determinants of returns for each cryptocurrency in the Current time period, ranging from 2017-01-04 to 2019-01-01

\begin{tabular}{|c|c|c|c|c|c|c|c|c|c|c|c|c|c|}
\hline & & LTC & XRP & XMR & $\mathrm{XLM}$ & $\mathrm{BCH}$ & BTC & ADA & ETH & NEO & EOS & TRX & ETC \\
\hline & Token used for & \multicolumn{5}{|c|}{ Transaction } & \multicolumn{3}{|c|}{ Hybrid } & \multicolumn{4}{|c|}{ Applications } \\
\hline H1 & circulating & & $n / a$ & & $n / a$ & & & $n / a$ & & $n / a$ & $n / a$ & $n / a$ & \\
\hline $\mathrm{H} 2$ & averagedifficulty & & $n / a$ & & $n / a$ & 0.0730 & & $\mathrm{n} / \mathrm{a}$ & 0.0054 & $n / a$ & $n / a$ & $n / a$ & 0.2264 \\
\hline H3 & exchangevolumeusd & & & & & & & & & 0.0090 & & & \\
\hline \multirow[b]{12}{*}{ H5 } & L.LreturnADA & & -0.0455 & & & & & $n / a$ & & & & & \\
\hline & L.LreturnBCH & & & & & $n / a$ & & & & -0.0013 & & & \\
\hline & \begin{tabular}{|l|} 
L.LreturnBTC \\
\end{tabular} & & -0.0168 & & & & $n / a$ & & & & & & \\
\hline & \begin{tabular}{|l|} 
L.LreturnEOS \\
\end{tabular} & & & & & & & & & & $n / a$ & & \\
\hline & \begin{tabular}{|l|} 
L.LreturnETC \\
\end{tabular} & & -0.0450 & & & & & & & & & -0.0667 & $n / a$ \\
\hline & \begin{tabular}{|l} 
L.LreturnETH \\
\end{tabular} & & & & & & & & $n / a$ & & & & \\
\hline & \begin{tabular}{|l} 
L.LreturnLTC \\
\end{tabular} & $\mathrm{n} / \mathrm{a}$ & & & & & & & & & 0.0530 & & \\
\hline & \begin{tabular}{|l|} 
L.LreturnNEO \\
\end{tabular} & & & & & & & & & $n / a$ & & & \\
\hline & \begin{tabular}{|l|} 
L.LreturnTRX \\
\end{tabular} & & 0.1056 & & & & & & & 0.0279 & & $n / a$ & \\
\hline & L.LreturnXLM & & & & $n / a$ & & & & & 0.0065 & & 0.0778 & \\
\hline & L.LreturnXMR & & & $n / a$ & & & & & & -0.0492 & & & \\
\hline & L.LreturnXRP & & $n / a$ & & & & & & -0.0086 & -0.0563 & -0.0399 & & \\
\hline H6 & google & & 0.0290 & & & & & & 0.0824 & 0.5983 & 0.6986 & & \\
\hline \multirow[b]{7}{*}{ H7 } & SP500 & & & & & & & & & & & & \\
\hline & NYSE & & & & & & & & & & & & \\
\hline & AMEX & & & & & & & & & 0.3361 & 0.0949 & & \\
\hline & NASDAQ & & & & & & & & & & & & \\
\hline & NIKKEI & & & & & & & & & & & & \\
\hline & SSE & & & & & & & & & & -0.1345 & & \\
\hline & Oil & & & & & & & & & & & & \\
\hline \multirow[b]{6}{*}{ H8 } & USEPU & & & & & & & & & & & & \\
\hline & EEPU & & 0.4699 & & & & & & & & & & \\
\hline & CEPU & & & & & & & & & -0.0743 & -0.1462 & & \\
\hline & VIX & & -0.0381 & & & & & & & & & & \\
\hline & VXo & & & & & & & & & & & & \\
\hline & VXN & & & & & & & & & 0.0436 & & & \\
\hline \multirow[b]{5}{*}{ H9 } & exchus & & & & & & & & & & & & \\
\hline & \begin{tabular}{|l} 
exjpus \\
\end{tabular} & & & & & & & & & & & & \\
\hline & exukus & & & & & & & & & & & & \\
\hline & exeuus & & & & & & & & & -0.5074 & -0.5168 & & \\
\hline & Gold & & & & & & & & & & & & \\
\hline \multirow{2}{*}{ Time trend } & Trend & & & & & & & & & & & & \\
\hline & Constant & 0.0002 & 0.0004 & -0.0009 & & -0.0018 & 0.0006 & 0.0021 & -0.0013 & -0.0021 & 0.0035 & 0.0042 & -0.0018 \\
\hline
\end{tabular}




\section{Appendix E: Unit root tests}

The Durbin Watson test examines if there exists a first-order serial correlation (i.e. autocorrelation at lag 1) in the residuals of a linear regression. The Durbin Watson d statistic can take on values between 0 and 4 and under the null $\mathrm{d}$ is equal to 2 , there is no autocorrelation. The null hypothesis of the Durbin Watson alternative test is that there is no first-order autocorrelation and the alternative hypothesis is that there exists autocorrelation. Values of $d$ less than 2 suggest positive autocorrelation whereas values of $d$ greater than 2 suggest negative autocorrelation. Table E1 displays the results, for all cryptocurrencies the Durbin-Watson d statistics are close to 2 , indicating that first-order residual autocorrelation is not a problem.

Table E1: McKinnon approximate d-statistic from Durbin Watson alternative test for serial correlation

\begin{tabular}{lll}
\hline Cryptocurrency & Abbreviation & d-statistic \\
\hline Cardano & ADA & 2,0086 \\
Bitcoin cash & BCH & 1,9257 \\
Bitcoin & BTC & 1,9451 \\
EOS & EOS & 2,0180 \\
Ethereum classic & ETC & 2,1582 \\
Ethereum & ETH & 2,0020 \\
Litecoin & LTC & 1,9438 \\
NEO & NEO & 2,0691 \\
Tron & TRX & 1,8814 \\
Stellar & XLM & 1,8939 \\
Monero & XMR & 2,1301 \\
Ripple & XRP & 1,9949 \\
\hline
\end{tabular}

The augmented Dickey Fuller (A.D.F.) test examines if a variable follows a unit root-process under the null hypothesis that the variable contains a unit root. The alternative hypothesis is that the variable was generated through a stationary process. The results of the A.D.F in Table E2 shows the differences before and after the log first difference transformation of the data. Before the transformation several variables have resulting p-values that exceed 0.05 (for a $5 \%$-significance level) which means that the null cannot be rejected, non-stationarity is a problem in the time series for the variable. After the transformation all variables fall below 0.05 , thus they are stationary after the log first difference transformation. 
Table E2: McKinnon approximate p-values for $Z(t)$ from A.D.F. test of stationarity

\begin{tabular}{|c|c|c|c|c|c|}
\hline \multirow[b]{2}{*}{ Cryptocurrency } & \multirow[b]{2}{*}{ Variable } & \multicolumn{2}{|c|}{ Before transformation } & \multicolumn{2}{|c|}{ After transformation } \\
\hline & & Dickey Fuller test & D.F. with trend & Dickey Fuller test & D.F. with trend \\
\hline \multirow[b]{3}{*}{ ADA } & exchangevolumeusd & 0 & 0 & 0 & 0 \\
\hline & return & 0 & 0 & 0 & 0 \\
\hline & google & 0,538 & 0,7442 & 0 & 0 \\
\hline \multirow[b]{5}{*}{$\mathrm{BCH}$} & circulating & 0,0014 & 0,7649 & 0 & 0 \\
\hline & averagedifficulty & 0,1968 & 0,4741 & 0 & 0 \\
\hline & exchangevolumeusd & 0 & 0 & 0 & 0 \\
\hline & return & 0 & 0 & 0 & 0 \\
\hline & google & 0,0213 & 0,06 & 0 & 0 \\
\hline \multirow[b]{5}{*}{ BTC } & circulating & 0 & 0 & 0 & 0 \\
\hline & averagedifficulty & 0,991 & 0,9885 & 0 & 0 \\
\hline & exchangevolumeusd & 0 & 0 & 0 & 0 \\
\hline & return & 0 & 0 & 0 & 0 \\
\hline & google & 0,709 & 0,8726 & 0 & 0 \\
\hline \multirow[b]{3}{*}{ EOS } & exchangevolumeusd & 0 & 0 & 0 & 0 \\
\hline & return & 0 & 0 & 0 & 0 \\
\hline & google & 0,3766 & 0,4747 & 0 & 0 \\
\hline \multirow[b]{5}{*}{ ETC } & circulating & 0 & 1 & 0 & 0 \\
\hline & averagedifficulty & 0,6397 & 0,711 & 0 & 0 \\
\hline & exchangevolumeusd & 0 & 0 & 0 & 0 \\
\hline & return & 0 & 0 & 0 & 0 \\
\hline & google & 0,3857 & 0,6082 & 0 & 0 \\
\hline \multirow[b]{5}{*}{ ETH } & circulating & 0 & 1 & 0 & 0 \\
\hline & averagedifficulty & 0,7446 & 0,9521 & 0 & 0 \\
\hline & exchangevolumeusd & 0 & 0 & 0 & 0 \\
\hline & return & 0 & 0 & 0 & 0 \\
\hline & google & 0,7048 & 0,9484 & 0 & 0 \\
\hline \multirow[b]{5}{*}{ LTC } & circulating & 0 & 0 & 0,0034 & 0 \\
\hline & averagedifficulty & 0,9991 & 0,9952 & 0 & 0 \\
\hline & exchangevolumeusd & 0 & 0 & 0 & 0 \\
\hline & return & 0 & 0 & 0 & 0 \\
\hline & google & 0,6987 & 0,8559 & 0 & 0 \\
\hline \multirow[b]{3}{*}{ NEO } & exchangevolumeusd & 0 & 0 & 0 & 0 \\
\hline & return & 0 & 0 & 0 & 0 \\
\hline & google & 0,9743 & 0,0874 & 0 & 0 \\
\hline & exchangevolumeusd & 0 & 0 & 0 & 0 \\
\hline & return & 0 & 0 & 0 & 0 \\
\hline TRX & google & 0,2296 & 0,5112 & 0 & 0 \\
\hline & exchangevolumeusd & 0 & 0 & 0 & 0 \\
\hline & return & 0 & 0 & 0 & 0 \\
\hline XLM & google & 0,2037 & 0,4592 & 0 & 0 \\
\hline & circulating & 0 & 0 & 0 & 0 \\
\hline & averagedifficulty & 0,2344 & 0,095 & 0 & 0 \\
\hline & exchangevolumeusd & 0 & 0 & 0 & 0 \\
\hline & return & 0 & 0 & 0 & 0 \\
\hline XMR & google & 0,7525 & 0,9639 & 0 & 0 \\
\hline & exchangevolumeusd & 0 & 0 & 0 & 0 \\
\hline & return & 0 & 0 & 0 & 0 \\
\hline XRP & google & 0,6156 & 0,8763 & 0 & 0 \\
\hline & SP550 & 0,8116 & 0,2143 & 0 & 0 \\
\hline & NYSE & 0,5424 & 0,3428 & 0 & 0 \\
\hline & AMEX & 0,9173 & 0,6425 & 0 & 0 \\
\hline & NASDAQ & 0,902 & 0,371 & 0 & 0 \\
\hline & NIKKEI & 0,4037 & 0,3237 & 0 & 0 \\
\hline & SSE & 0,4097 & 0,781 & 0 & 0 \\
\hline & Oil & 0,3047 & 0,9503 & 0 & 0 \\
\hline & USEPU & 0,0455 & 0,0015 & 0 & 0 \\
\hline & EEPU & 0,8511 & 0,953 & 0 & 0 \\
\hline & CEPU & 0,8905 & 0,8895 & 0 & 0 \\
\hline & VIX & 0 & 0 & 0 & 0 \\
\hline & $\mathrm{VXO}$ & 0 & 0 & 0 & 0 \\
\hline & VXN & 0 & 0 & 0 & 0 \\
\hline & exchus & 0,7015 & 0,9229 & 0 & 0 \\
\hline & exjpus & 0,1932 & 0,544 & 0 & 0 \\
\hline Not & exukus & 0,6532 & 0,7135 & 0 & 0 \\
\hline cryptocurrency & exeuus & 0,3991 & 0,816 & 0 & 0 \\
\hline specific variables & Gold & 0,0807 & 0,1982 & 0 & 0 \\
\hline
\end{tabular}

